\title{
ESTIMATES FOR THE HEAT KERNEL ON DIFFERENTIAL FORMS ON RIEMANNIAN SYMMETRIC SPACES AND APPLICATIONS *
}

\author{
N. LOHOUÉ ${ }^{\dagger}$ AND S. MEHDI
}

\begin{abstract}
We prove upper bounds estimates for the large time behavior of the heat kernel and for the resolvent of the form Laplacian on Riemannian symmetric spaces, and we obtain $L^{2+\epsilon}-$ estimates for its resolvent on locally symmetric spaces. We deduce lower bounds for the bottom of the spectrum of the form Laplacian and some results on the vanishing of the $L^{2}$-cohomology of locally symmetric spaces.
\end{abstract}

Key words. Heat kernel, Form Laplacian, Riemannian symmetric space, locally symmetric space, Plancherel formula, Paley-Wiener theorem, $L^{2}$ - cohomology, principal series representation, isotropy representation.

AMS subject classifications. Primary 22E46, 43A85, 53C35, 58J35, 58J50; Secondary 22E40, $57 \mathrm{~T} 15$

1. Introduction. In the last decades the heat kernel has become a fundamental and powerful tool, subject of a rich and vast literature, reflecting its universality and formidable efficiency: Atiyah-Singer index theory, K-theory, spectral geometry, zeta and theta functions, $L^{2}$-invariants, anomalies, quantum gravity, ... (see e.g. [4], [7], [28], [37]). Despite these tremendous advances, explicit estimates for the asymptotics of the heat kernel and computation of related $L^{2}$-invariants are not available in general. However, for a large class of Riemannian manifolds, representation theoretic techniques may be used to obtain estimates for the asymptotics, compute $L^{2}$-invariants and derive some results on the $L^{2}$-cohomology.

More precisely, let $G$ be a non compact connected semisimple Lie group with finite center and $K$ a maximal compact subgroup of $G$. The homogeneous space $G / K$ is naturally equipped with a structure of a non compact Riemannian symmetric manifold, the metric being induced by the Killing form of $G$. A finite dimensional representation $(\tau, E)$ of $K$ induces a homogeneous vector bundle $\mathcal{E}$ over $G / K$. The group $G$ acts by left translations on the Hilbert space $L^{2}(G / K, \mathcal{E})$ of square integrable sections of $\mathcal{E}$. Let

$$
D: L^{2}(G / K, \mathcal{E}) \rightarrow L^{2}(G / K, \mathcal{E})
$$

be a $G$-invariant selfadjoint positive elliptic operator, i.e $D$ commutes with the action of $G$ on $L^{2}(G / K, \mathcal{E})$. Denote by $P_{t}=e^{-t D}$ the fundamental solution of the heat equation

$$
\left\{\begin{array}{l}
D P_{t}=-\frac{\partial}{\partial t} P_{t}, \quad t>0 \\
P_{0}=\delta
\end{array}\right.
$$

where $\delta$ is the Dirac function. In particular, for each $\psi$ in $L^{2}(G / K, \mathcal{E})$, the convolution

\footnotetext{
*Received May 30, 2009; accepted for publication October 4, 2010.

†UMR 8628-CNRS and Département de mathématiques, Université Paris Sud-Orsay, France (Noel.Lohoue@math.upsud.fr).

‡UMR 7122-CNRS and Département de mathématiques, Université Paul Verlaine-Metz, France (mehdi@univ-metz.fr).
} 
product $P_{t} \star \psi$ is a solution of the heat equation in $L^{2}(G / K, \mathcal{E})$

$$
\left\{\begin{array}{l}
D \phi_{t}=-\frac{\partial}{\partial t} \phi_{t}, t>0 \\
\phi_{0}=\psi
\end{array}\right.
$$

It is known (see [6]) that the heat operator $P_{t}=e^{-t D}$ is a bounded operator on $L^{2}(G / K, \mathcal{E})$ so that

$$
\left(P_{t} f\right)(g)=\int_{G} \mathcal{P}_{t}\left(g, g^{\prime}\right) f\left(g^{\prime}\right) d g^{\prime}, \quad \forall f \in L^{2}(G / K, \mathcal{E})
$$

where

$$
\mathcal{P}_{t}: G \times G \rightarrow \operatorname{End}(E)
$$

is the heat kernel, and $\operatorname{End}(E)$ denotes the vector space of complex endomorphisms of $E$.

On the other hand, a torsion free discrete subgroup $\Gamma$ of $G$ acts on the left on $G / K$, so that the double coset space $\Gamma \backslash G / K$ is a locally symmetric Riemannian manifold. Except otherwise stated, we assume that $\Gamma$ is not of finite covolume in $G$. Since $G / K$ is simply connected, it is the universal cover of $\Gamma \backslash G / K$ and

$$
\Gamma \simeq \Pi_{1}(\Gamma \backslash G / K) .
$$

The bundle $\mathcal{E}$ can be pushed down to a bundle over $\Gamma \backslash G / K$ and we let $L^{2}(\Gamma \backslash G / K, \mathcal{E})$ denote the corresponding Hilbert space of square integrable sections. In particular, the operator $D$ drops down to $\Gamma \backslash G / K$ and defines a locally invariant selfadjoint positive elliptic operator

$$
\widetilde{D}: L^{2}(\Gamma \backslash G / K, \mathcal{E}) \rightarrow L^{2}(\Gamma \backslash G / K, \mathcal{E}) .
$$

Write respectively $\widetilde{P}_{t}$ and $\widetilde{\mathcal{P}}_{t}$ for the corresponding heat operator and heat kernel. In this setting a certain pair $(D, \mathcal{E})$ will be distinguished. This particular pair, which we will focus on, may be thought of as a fundamental model for the general theory.

Consider the Cartan decomposition

$$
\mathfrak{g}=\mathfrak{k} \oplus \mathfrak{s}
$$

of $\mathfrak{g}$, where $\mathfrak{g}$ (resp. $\mathfrak{k}$ ) is the complexification of the Lie algebra of $G$ (resp. $K$ ) and $\mathfrak{s}$ is a complex vector subspace of $\mathfrak{g}$ satisfying the bracket relations

$$
[\mathfrak{k}, \mathfrak{s}] \subset \mathfrak{s} \text { and }[\mathfrak{s}, \mathfrak{s}] \subset \mathfrak{k} \text {. }
$$

The adjoint representation of $G$ induces a finite dimensional representation $\sigma_{\ell}$ of $K$ on the exterior product

$$
V_{\ell}=\Lambda^{\ell} \mathfrak{s}
$$

of $\mathfrak{s}$, for $\ell=0, \cdots, \operatorname{dim}(\mathfrak{s})$, known as the isotropy representation. It should be noted that the decomposition of $\sigma_{\ell}$ into irreducible components is not known in general, except for (real) rank one groups [13]. We have made some explicit computations for real groups with (real) rank two and for complex groups. These results are described in 
the appendix. From now on we choose the representation $(\tau, E)$ to be the isotropy representation of $K$. In particular the Hilbert space $L^{2}(G / K, \mathcal{E})\left(\right.$ resp. $\left.L^{2}(\Gamma \backslash G / K, \mathcal{E})\right)$ identifies naturally with the space $L^{2}\left(G / K, \mathcal{V}_{\ell}\right)$ (resp. $L^{2}\left(\Gamma \backslash G / K, \mathcal{V}_{\ell}\right)$ ) of square integrable $\ell$-forms on $G / K$ (resp. $\Gamma \backslash G / K$ ). Then we take $D$ (resp. $\widetilde{D}$ ) to be the Hodge-de Rham Laplacian $\Delta_{\ell}$ (resp. $\widetilde{\Delta}_{\ell}$ ) acting on $\ell$-forms, with corresponding heat kernel $\mathcal{P}_{t}^{\ell}$ (resp. $\widetilde{\mathcal{P}}_{t}^{\ell}$ ). Related to the large time behavior of the heat kernel, there are several interesting $L^{2}$-invariants of $\Gamma \backslash G / K$ which can be computed explicitly [33]. One of these invariants will bear some special interest to us. Let $\Delta_{\ell}^{\perp}$ be the restriction of $\Delta_{\ell}$ to the orthogonal complement of $\operatorname{Ker}\left(\Delta_{\ell}\right)$ in $L^{2}\left(G / K, \mathcal{V}_{\ell}\right)$. The $\Gamma$-trace of the corresponding heat kernel $\mathcal{P}_{t}^{\ell, \perp}$ is defined as follows:

$$
\operatorname{Tr}_{\Gamma}\left(\mathcal{P}_{t}^{\ell, \perp}\right)=\int_{\mathcal{F}} \operatorname{Tr}\left(\mathcal{P}_{t}^{\ell, \perp}(x, x)\right) d x
$$

where $\mathcal{F} \subset G / K$ is a fundamental domain for the action of $\Gamma$ on $G / K$. Then the $\ell$ th Novikov-Shubin invariant of $\Gamma \backslash G / K$ is given by

$$
a_{\ell}(\Gamma \backslash G / K)=\sup \left\{b \in \mathbf{R}_{+} \mid \operatorname{Tr}_{\Gamma}\left(\mathcal{P}_{t}^{\ell, \perp}\right) \stackrel{t \rightarrow+\infty}{=} O\left(t^{-\frac{b}{2}}\right)\right\} .
$$

This value (possibly infinite), which does not depend on $\Gamma$, nor on the Riemannian metric on $\Gamma \backslash G / K$, measures the asymptotic behavior of the spectral density function of $\Delta_{\ell}$ at 0 . Roughly speaking the $\ell$ th Novikov-Shubin invariant measures the thickness of the spectrum of $\Delta_{\ell}$ near 0 . Using a Plancherel formula for differential forms, we have computed, for $\Gamma$ of finite covolume, explicitly these invariants in [26] (see [33] for a more complete discussion on $L^{2}$-invariants of locally symmetric spaces):

$$
a_{\ell}(\Gamma \backslash G / K)=\left\{\begin{array}{l}
\operatorname{rk}_{\mathbf{C}}(G)-\operatorname{rk}_{\mathbf{C}}(K) \text { if } \ell \in I(G ; K) \text { and } \operatorname{rk}_{\mathbf{C}}(G)>\operatorname{rk}_{\mathbf{C}}(K) \\
\infty^{+} \text {otherwise }
\end{array}\right.
$$

where $\operatorname{rk}_{\mathbf{C}}(G)$ (resp. $\left.\operatorname{rk}_{\mathbf{C}}(K)\right)$ denotes the complex rank of $G$ (resp. $\left.K\right)$ and $I(G ; K)$ is the interval $\left[\frac{1}{2} \operatorname{dim}_{\mathbf{R}}(G / K)-\frac{1}{2}\left(\operatorname{rk}_{\mathbf{C}}(G)-\operatorname{rk}_{\mathbf{C}}(K)\right), \frac{1}{2} \operatorname{dim}_{\mathbf{R}}(G / K)+\frac{1}{2}\left(\operatorname{rk}_{\mathbf{C}}(G)-\right.\right.$ $\left.\operatorname{rk}_{\mathbf{C}}(K)\right)$.

In the sequel we shall focus on the continuous part of the heat kernel. More precisely, let $\mathcal{P}_{t}^{\ell, \perp}$ be the heat kernel associated with $\Delta_{\ell}^{\perp}$, i.e the projection of $\mathcal{P}_{t}^{\ell}$ onto the restriction of $\Delta_{\ell}$ to the orthogonal complement of $\operatorname{Ker}\left(\Delta_{\ell}\right)$ in $L^{2}\left(G / K, \mathcal{V}_{\ell}\right)$. It turns out that by a result of Borel (2.7), $\mathcal{P}_{t}^{\ell}$ and $\mathcal{P}_{t}^{\ell, \perp}$ coincide when $\operatorname{rk}_{\mathbf{C}}(G)>\operatorname{rk}_{\mathbf{C}}(K)$ or when $\operatorname{rk}_{\mathbf{C}}(G)=\operatorname{rk}_{\mathbf{C}}(K)$ and $\ell \neq \frac{1}{2} \operatorname{dim}_{\mathbf{R}}(G / K)$.

We now turn to the statement of our main results.

TheOREm 1 (Theorem 3.1). For all $\epsilon \in] 0,1\left[\right.$ there exist two positive numbers $a_{\epsilon}$ and $A_{\epsilon}$ such that, for all $g \in G$ and $t \in \mathbf{R}$ satisfying $\|g\|>A_{\epsilon}$ and $t>1$, we have

$$
\left\|\mathcal{P}_{t}^{\ell, \perp}(g)\right\| \leq a_{\epsilon} e^{-t \lambda_{\ell}(G / K)} \Phi_{0}(g) e^{-\frac{1-\epsilon}{(1+2 \epsilon)^{2}} \frac{\|g\|^{2}}{4 t}} t^{-\epsilon \frac{r+z}{2}}
$$

where $\lambda_{\ell}(G / K)$ is the bottom of the spectrum of $\Delta_{\ell}, \Phi_{0}$ is the Harish-Chandra spherical function on $G, r$ is the minimal dimension of non trivial split components of cuspidal parabolic subgroups of $G$ and $z$ is the minimum of the orders of zero of the Harish-Chandra c-functions corresponding to the conjugacy classes of proper cuspidal parabolic subgroups of $G$.

The strategy of the proof is 
- use the expression of $\mathcal{P}_{t}^{\ell, \perp}$ derived from the Plancherel formula for square integrable $\ell$-forms on $G / K$,

- following an idea of Alexopoulos and Lohoué [2], we decompose the scalar product $\left\langle p_{t}^{\ell, \perp}(g) \eta, \beta\right\rangle_{\Lambda^{\ell_{\mathfrak{S}}} \mathrm{C}}, g \in G$, in two pieces $\varphi_{1, \epsilon}(g)$ and $\varphi_{2, \epsilon}(g)$ with support depending on $\epsilon$, observing that we can choose $\eta$ and $\beta$ in the same irreducible component of $\sigma_{\ell}$,

- combine a recent result of van den Ban and Souaifi on the proof by Delorme of a Paley-Wiener Theorem on the group $G$, to see that $\varphi_{2, \epsilon}$ is smooth and compactly supported, whose support does not contain $g$,

- estimate $\varphi_{1, \epsilon}$.

A link between the power of $t$ in the above estimate and the $\ell$ th Novikov-Shubin invariant of $\Gamma \backslash G / K$ is provided by Corollary 3.10.

TheOREM 2 (Theorem 4.1). For all $\epsilon \in] 0,1\left[\right.$, there exist two positive numbers $b_{\epsilon}$ and $B_{\epsilon}$ such that, for all $g \in G$ satisfying $\|g\|>B_{\epsilon}$, we have

$$
\left\|\left(\Delta_{\ell}-\mu\right)^{-1}(g)\right\| \leq b_{\epsilon} \Phi_{0}(g) e^{-(1-\epsilon) \tau_{\mu, \ell}(G / K)\|g\|}
$$

where $\mu$ is a complex number in the resolvent set of $\Delta_{\ell}$ and $\tau_{\mu, \ell}(G / K)$ is some positive real number depending on $\mu$ and on the bottom of the spectrum of $\Delta_{\ell}$.

The main steps of the proof are

- estimate the convolution product $\left(\Delta_{\ell}-\mu\right)^{-1} \star P_{\epsilon_{0}}^{\ell}$ for $\epsilon_{0}$ sufficiently small,

- prove that the constants involved in our estimates do not depend on $\epsilon_{0}$,

- take the limit $\epsilon_{0} \rightarrow 0$.

In the case of functions, i.e when $\ell=0$, sharp estimates for the heat kernel $\mathcal{P}_{t}^{\ell}$ and the resolvent of $\Delta_{\ell}$ were obtained by J.-P. Anker and L. Ji in [3].

TheOREM 3 (Theorem 5.9). Assume that $\Gamma$ is of finite covolume in $G$. Then for all complex number $\mu$ with positive imaginary part and element $\dot{g} \in \Gamma \backslash G$, there exists a positive number $\epsilon$ such that $\left(\widetilde{\Delta}_{\ell}-\mu\right)^{-k}(\dot{g}, \cdot)$ belongs to $L^{2+\epsilon}\left(\Gamma \backslash G / K, \mathcal{E} n d\left(\Lambda^{\ell} \mathfrak{s}\right)\right)$, for all integer $k>\frac{1}{4} \operatorname{dim}_{\mathbf{R}}(G / K)$.

The main lines of the proof are

- recall, by a result of $A$. Borel and H. Garland, that the kernel $\operatorname{Ker}\left(\widetilde{\Delta}_{\ell}\right)$ of $\widetilde{\Delta}_{\ell}$ is finite dimensional,

- use a result of $\mathrm{N}$. Lohoué on the stability of $L^{p}$-cohomology around 2 to show that the orthogonal projection $T_{\ell}: L^{2}\left(\Gamma \backslash G / K, \Lambda^{\ell} \mathfrak{s}\right) \rightarrow \operatorname{Ker}\left(\widetilde{\Delta}_{\ell}\right)$ is a bounded operator on $L^{2+\epsilon}\left(\Gamma \backslash G / K, \Lambda^{\ell} \mathfrak{s}\right)$ for some positive real number $\epsilon$,

- use a Stein interpolation theorem to prove that $\left(\widetilde{\Delta}_{\ell}-\mu\right)^{-1}$ is a bounded operator on $L^{p}\left(\Gamma \backslash G / K, \Lambda^{\ell} \mathfrak{s}\right)$ for $p \in[2,2+\epsilon]$ and $\operatorname{Im}(\mu)$ sufficiently large,

- analyze the generic terms occuring in the $k$ th power of $\left(\widetilde{\Delta}_{\ell}-\mu\right)^{-1}$, with $k>\frac{1}{4} \operatorname{dim}_{\mathbf{R}}(G / K)$.

Note that this theorem, combined with Proposition 5.5, generalizes a result of $\mathrm{R}$. Miatello and N. Wallach proved for functions, i.e when $\ell=0$, in the case where $G$ has real rank one (Theorem 3.4 in [30]).

ThEOREM 4 (Theorem 6.1). Let $\beta_{\ell}(\Gamma \backslash G / K)$ be the bottom of the spectrum of $\widetilde{\Delta}_{\ell}$ and $\delta(\Gamma)$ the critical exponent of $\Gamma$. We assume that $\Gamma$ is of infinite covolume in $G$. Let $\rho$ be the half sum of positive restricted $\mathfrak{g}$-roots and $\rho_{\min }$ the minimum of the 
values $\rho(X),\|X\|=1$, taken on the closure of a positive Weyl chamber in $\mathfrak{s}$. If we assume that $\lambda_{\ell}(G / K)$ does not vanish, then we have

(i) if $\delta(\Gamma) \leq \rho_{\text {min }}$ then $\beta_{\ell}(\Gamma \backslash G / K) \geq \lambda_{\ell}(G / K)$,

(ii) if $\rho_{\min } \leq \delta(\Gamma) \leq\|\rho\|+\sqrt{\lambda_{\ell}(G / K)}$ then $\beta_{\ell}(\Gamma \backslash G / K) \geq \lambda_{\ell}(G / K)-(\delta(\Gamma)-$ $\left.\rho_{\text {min }}\right)^{2}$, and

(iii) if $\left|\delta(\Gamma)-\rho_{\text {min }}\right| \leq\|\rho\|<\delta(\Gamma)$ and $\lambda_{\ell}(G / K) \geq\left(\delta(\Gamma)-\rho_{\text {min }}\right)^{2}$ then $\beta_{\ell}(\Gamma \backslash G / K) \geq \lambda_{\ell}(G / K)-\left(\delta(\Gamma)-\rho_{\text {min }}\right)^{2}$.

The main idea of the proof is

- use Poincaré series to deduce, from the previous theorem, an estimate for the resolvent of $\widetilde{\Delta}_{\ell}$

- combine this estimate with some recent result of E. Leuzinger on $\beta_{0}(\Gamma \backslash G / K)$. An immediate consequence on the $L^{2}$-cohomology of $\Gamma \backslash G / K$ can be deduced (see Section 2.13 for definitions).

Corollary (Corollary 6.5). The (reduced or unreduced) $L^{2}$-cohomology group of degree $\ell$ of $\Gamma \backslash G / K$ is trivial in the following cases:

(i) $\delta(\Gamma) \leq \rho_{\min }$,

(ii) $\rho_{\min } \leq \delta(\Gamma) \leq\|\rho\|+\sqrt{\lambda_{\ell}(G / K)}$ and $\sqrt{\lambda_{\ell}(G / K)}>\delta(\Gamma)-\rho_{\min }$,

(iii) $\left|\delta(\Gamma)-\rho_{\min }\right| \leq\|\rho\|<\delta(\Gamma)$ and $\sqrt{\lambda_{\ell}(G / K)}>\left|\delta(\Gamma)-\rho_{\min }\right|$.

In particular, in these cases, the kernel of $\widetilde{\Delta}_{\ell}$ is reduced to $\{0\}$.

Analogous results for hyperbolic manifolds were obtained by G. Caron and E. Pedon in [13].

Our paper is organized as follows: in Section 2, we fix notations, recall some facts and give a representation theoretic description of the bottom of the spectrum of $\Delta_{\ell}$ (proposition 2.32). Section 3 (resp. Section 4) is devoted to the proof of upper bounds estimates for the large time behavior of the heat kernel (resp. resolvent) of $\Delta_{\ell}$. Section 5 contains a proof of an $L^{2+\epsilon}$-estimate for the resolvent of $\widetilde{\Delta}_{\ell}$. In Section 6 , we give lower bounds for the bottom of the spectrum of $\widetilde{\Delta}_{\ell}$ and we deduce some results on the vanishing of the (reduced or unreduced) $L^{2}$-cohomology of $\Gamma \backslash G / K$. Finally we have gathered in the appendix some computations on the bottom of the spectrum of $\Delta_{\ell}$. The main results in this paper were announced without proof in [25].

Acknowledgements. We thank Erik van den Ban for providing us with some new insights on Delorme's Paley-Wiener theorem which helped us to fill a gap in a first version of the paper. The second named author is indebted to Martin Olbrich for useful conversations. We also thank the referee for comments and suggestions that helped us improve the paper.

\section{Preliminaries.}

2.1. Roots, decompositions and norms. Let $G$ be a non compact connected semisimple real Lie group with finite center and Lie algebra $\mathfrak{g}_{0}$. Fix a Cartan involution $\Theta$ of $G$ and let $K$ be the corresponding maximal compact subgroup of $G$ with Lie algebra $\mathfrak{k}_{0}$. We shall drop the subscript 0 for the complexification. Let $\theta$ be the Cartan involution of $\mathfrak{g}_{0}$ derived from $\Theta$ and let

$$
\mathfrak{g}_{0}=\mathfrak{k}_{0} \oplus \mathfrak{s}_{0}
$$

be the associated Cartan decomposition. There is a finite number $s$ of conjugacy classes of $\theta$-stable Cartan subalgebras in $\mathfrak{g}_{0}$, so we fix an element $\mathfrak{h}_{i, 0}$ in each class 
and we put

$$
\mathfrak{a}_{i, 0}=\mathfrak{h}_{i, 0} \cap \mathfrak{s}_{0} \text { and } \mathfrak{t}_{i, 0}=\mathfrak{h}_{i, 0} \cap \mathfrak{k}_{0} .
$$

Let $\Delta_{i}$ be the set of $\mathfrak{g}$-roots relative to $\mathfrak{h}_{i}$ and fix a system of positive roots $\Delta_{i}^{+} \subset \Delta_{i}$. Write $\Sigma_{i}$ for the set of restricted roots, i.e the set of $\mathfrak{g}_{0}$-roots with respect to $\mathfrak{a}_{i, 0}$. Choose a system $\Sigma_{i}^{+} \subset \Sigma_{i}$ of positive restricted roots with the compatibility condition

$$
\left.\left(\alpha \in \Delta_{i}^{+} \text {and }\left.\alpha\right|_{\mathfrak{a}_{i, 0}} \neq 0\right) \Longrightarrow \alpha\right|_{\mathfrak{a}_{i, 0}} \in \Sigma_{i}^{+}
$$

As usual write

$$
\rho_{i}=\frac{1}{2} \sum_{\alpha \in \Delta_{i}^{+}} \alpha
$$

for the half-sum of positive roots and

$$
\rho_{\mathfrak{a}_{i, 0}}=\frac{1}{2} \sum_{\alpha \in \Sigma_{i}^{+}} m_{\alpha} \alpha
$$

for the half-sum of positive restricted roots counted with their multiplicities, i.e $m_{\alpha}=\operatorname{dim}\left(\mathfrak{g}_{0}\right)_{\alpha}$, where $\left(\mathfrak{g}_{0}\right)_{\alpha}$ denotes the root space corresponding to $\alpha$. The subset $\Sigma_{i}^{++}$of $\Sigma_{i}^{+}$will denote the set of positive indivisible restricted roots

$$
\Sigma_{i}^{++}=\left\{\alpha \in \Sigma_{i}^{+} \mid \frac{1}{2} \alpha \notin \Sigma_{i}^{+}\right\} .
$$

Write $\operatorname{rk}_{\mathbf{C}}(G)$ and $\operatorname{rk}_{\mathbf{C}}(K)$ for the complex ranks of $G$ and $K$ respectively. Denote by $W_{i}$ the Weyl group associated with $\Delta_{i}$ and by $\left|W_{i}\right|$ its order. Let

$$
\mathfrak{n}_{i, 0}=\sum_{\alpha \in \Sigma_{i}^{+}}\left(\mathfrak{g}_{0}\right)_{\alpha} \text { and } \mathfrak{m}_{i, 0}=\mathfrak{t}_{i, 0}+\sum_{\beta \in \Delta_{i},\left.\beta\right|_{\mathfrak{a}_{i, 0}}=0}\left(\mathfrak{g}_{0}\right)_{\beta} .
$$

Write $\left(M_{i}\right)_{e}, A_{i}$ and $N_{i}$ for the analytic subgroups of $G$ with Lie algebra $\mathfrak{m}_{i, 0}, \mathfrak{a}_{i, 0}$ and $\mathfrak{n}_{i, 0}$ respectively. There exists a unique $\Theta$-stable subgroup $M_{i}$ of $G$ such that the centralizer of $\mathfrak{a}_{i, 0}$ in $G$ is $M_{i} A_{i}$. The subgroup

$$
P_{i}=M_{i} A_{i} N_{i}
$$

is a cuspidal parabolic subgroup of $G$, in particular the discrete series $\left(\widehat{M}_{i}\right)_{d}$ of $M_{i}$ is not empty. We may describe, in this way, the set of all conjugacy classes of cuspidal parabolic subgroups of $G$ with Lie algebra

$$
\mathfrak{p}_{i, 0}=\mathfrak{m}_{i, 0} \oplus \mathfrak{a}_{i, 0} \oplus \mathfrak{n}_{i, 0} .
$$

Observe that the group $G$ itself is cuspidal if, and only if, the discrete series $\widehat{G}_{d}$ of $G$ is not empty, i.e $\operatorname{rk}_{\mathbf{C}}(G)=\operatorname{rk}_{\mathbf{C}}(K)$.

We shall drop the subscript $i$ and simply write $\mathfrak{a}_{0}$ for a maximal abelian subspace of $\mathfrak{s}_{0}, \mathfrak{a}$ its complexification, $\Sigma$ the set of restricted roots, $\rho_{\mathfrak{a}_{0}}$ the half-sum of positive restricted roots and $P=M A N$ the corresponding (minimal) parabolic subgroup of $G$. The real $\operatorname{rank} \operatorname{rk}_{\mathbf{R}}(G)$ of $G$ is the dimension of $\mathfrak{a}_{0}$. The Iwasawa decomposition of $G$ is

$$
G=K A N
$$


where any element $g$ of $G$ can be written in a unique way as

$$
g=k(g) e^{a(g)} n(g) .
$$

Moreover our choice of $\Sigma^{+}$fixes a positive Weyl chamber $\mathfrak{a}_{0}^{+}$in $\mathfrak{a}_{0}$ which defines the following Cartan decomposition of $G$

$$
G=K \exp \left(\overline{\mathfrak{a}_{0}^{+}}\right) K
$$

where any element $g$ of $G$ can be written as

$$
g=k_{1}(g) e^{a^{+}(g)} k_{2}(g),
$$

$\overline{\mathfrak{a}_{0}^{+}}$being the closure of $\mathfrak{a}_{0}^{+}$. Note that the component $a^{+}(g)$ of $g$ is uniquely determined, whereas the $K$-components $k_{1}(g)$ and $k_{2}(g)$ are not.

The Killing form of $\mathfrak{g}$

$$
\mathcal{K}: \mathfrak{g} \times \mathfrak{g} \rightarrow \mathbf{C},(X, Y) \mapsto \operatorname{Tr}(\operatorname{ad}(X) \circ \operatorname{ad}(Y))
$$

defines the following $G$-invariant inner product on $\mathfrak{g}$

$$
\langle X, Y\rangle=-\mathcal{K}(X, \theta(Y))
$$

which in turn induces a Riemannian structure on the symmetric space $G / K$, whose tangent space at the origin $e K$ is identified with $\mathfrak{s}$. In particular, this enables us to identify $\mathfrak{g}$ with its vector dual $\mathfrak{g}^{\star}$, as well as subspaces of $\mathfrak{g}$ with subspaces in $\mathfrak{g}^{\star}$. We shall denote by the same symbol \|\| the induced norms on $\mathfrak{g}$ and $\mathfrak{g}^{\star}$, as well as the norm on $G$ defined by

$$
\|g\|=\left\|a^{+}(g)\right\| \text {. }
$$

In particular one has

$$
\left\|g^{-1}\right\|=\|g\| \text { and }\left\|k g k^{\prime}\right\|=\|g\| \text { for all } g \in G \text { and } k, k^{\prime} \in K \text {. }
$$

2.2. Principal series representations. Fix a proper parabolic subgroup $P_{i}=$ $M_{i} A_{i} N_{i}$ of $G$. Let $\delta_{i}$ be a discrete series representation of $M_{i}$ in some Hilbert space $V_{\delta_{i}}$ equipped with an $M_{i}$-invariant scalar product $\langle,\rangle_{V_{\delta_{i}}}$ and an induced norm \|\|$_{V_{\delta_{i}}}$. Let $\alpha_{i}$ be a linear form on $\mathfrak{a}_{i}$. The principal series representation of $G$ associated with the data $P_{i}, \delta_{i}$ and $\alpha_{i}$ is the induced representation

$$
\pi_{P_{i}, \delta_{i}, \alpha_{i}} \stackrel{\text { def. }}{=} \operatorname{Ind}_{P_{i}}^{G}\left(\delta_{i} \otimes e^{\alpha_{i}+\rho_{a_{i, 0}}} \otimes 1\right)
$$

of $G$ in some Hilbert space $\mathcal{H}_{P_{i}, \delta_{i}, \alpha_{i}}$. More precisely, write $V_{\delta_{i}}^{\infty}$ for the space of smooth vectors in $\delta_{i}$ and consider the vector space $\mathcal{H}_{P_{i}, \delta_{i}, \alpha_{i}}^{\infty}$ of $V_{\delta_{i}}^{\infty}$-valued smooth functions on $G$ satisfying the equivariance relation

$$
f(\text { gman })=e^{-\left(\alpha_{i}+\rho_{a_{i, 0}}\right)(a)} \delta_{i}(m)^{-1}(f(g)) \forall g \in G, m \in M_{i}, a \in A_{i}, n \in N_{i}
$$

equipped with the scalar product

$$
\left\langle\phi_{1}, \phi_{2}\right\rangle_{\mathcal{H}_{P_{i}, \delta_{i}, \alpha_{i}}}=\int_{K}\left\langle\phi_{1}(k), \phi_{2}(k)\right\rangle_{V_{\delta_{i}}} d k .
$$


Then the Hilbert space $\mathcal{H}_{P_{i}, \delta_{i}, \alpha_{i}}$ is the completion of $\mathcal{H}_{P_{i}, \delta_{i}, \alpha_{i}}^{\infty}$ with respect to this norm on which $G$ acts by left translations. If $\alpha_{i}$ is imaginary then $\pi_{P_{i}, \delta_{i}, \alpha_{i}}$ is a unitary representation (Chapter VII of [20]). The module of smooth vectors of the principal series representation $\pi_{P_{i}, \delta_{i}, \alpha_{i}}$ of $G$ has a realization in the space $C^{\infty}\left(K ; \delta_{i}\right)$ of smooth $V_{\delta_{i}}$-valued maps $\phi: K \longrightarrow V_{\delta_{i}}$ on $K$ transforming under the rule

$$
\phi(k m)=\delta_{i}^{-1}(m) \phi(k) \quad \forall k \in K, m \in K \cap M_{i} .
$$

If $g \in G$ decomposes under $G=K M_{i} A_{i} N_{i}$ as

$$
g=\kappa(g) \mu(g) e^{H(g)} n
$$

then the action of $G$ on $C^{\infty}\left(K ; \delta_{i}\right)$ is given by

$$
\pi_{P_{i}, \delta_{i}, \alpha_{i}}(g) \phi(k)=e^{-\left(\alpha_{i}+\rho_{a_{i, 0}}\right)\left(H\left(g^{-1} k\right)\right)} \delta_{i}\left(\kappa\left(g^{-1} k\right)\right)^{-1} \phi\left(\kappa\left(g^{-1} k\right)\right) .
$$

This is known as the compact picture realization of the principal series representation $\pi_{P_{i}, \delta_{i}, \alpha_{i}}$ (chapter VII of [20]).

2.3. $L^{p}$-integrable differential forms on $G / K$. The group $K$ acts on $\mathfrak{s}$ by the restriction of the (linear extension of the) adjoint action Ad of $G$. This action induces a representation $\sigma_{\ell}$ of $K$ on the exterior product $V_{\ell}=\wedge^{\ell} \mathfrak{s}$

$$
\begin{aligned}
\sigma_{\ell}(k)\left(v_{1} \wedge v_{2} \wedge \cdots \wedge v_{\ell}\right) & =\operatorname{Ad}(k) v_{1} \wedge \operatorname{Ad}(k) v_{2} \cdots \wedge \operatorname{Ad}(k) v_{\ell}, \quad \ell \geq 1, \\
\sigma_{0}(k) v & =v, \quad v \in \mathbf{C},
\end{aligned}
$$

known as the isotropy representation. Observe that, by Hodge isomorphism, $\sigma_{\ell}$ and $\sigma_{\operatorname{dim}(\mathfrak{s})-\ell}$ are equivalent for all $0 \leq \ell \leq \operatorname{dim}(\mathfrak{s})$. The isotropy representation is not irreducible in general, and its explicit decomposition into irreducibles

$$
\left(\sigma_{\ell}, V_{\ell}\right) \simeq \bigoplus\left(\sigma_{\ell}^{j}, V_{\ell}^{j}\right)
$$

is still an open problem. We fix a $K$-invariant scalar product $\langle,\rangle_{\Lambda^{\ell} \mathfrak{s}}$ on $V_{\ell}$ such that

$$
\left\langle V_{\ell}^{i}, V_{\ell}^{j}\right\rangle=\{0\} \text { if } i \neq j .
$$

The isotropy representation defines a homogeneous vector bundle $\mathcal{V}_{\ell}$ over $G / K$ and we let $L^{p}\left(G / K, \mathcal{V}_{\ell}\right)$ be the space of its $L^{p}$-sections, i.e the $L^{p}$-integrable $\ell$-forms on $G / K$, with $p \in \mathbf{N}^{\star}$. Naturally there is an action, by left translations, of the group $G$ on $L^{p}\left(G / K, \mathcal{V}_{\ell}\right)$. More precisely, the tensor product $L^{p}(G) \otimes \wedge^{\ell} \mathfrak{s}$ is equipped with an action of $G$ and of $K$ given respectively by $L \otimes \mathbb{1}$ and $R \otimes \sigma_{\ell}$, where $L^{p}(G)$ is the space of $L^{p}$-integrable complex functions on $G$ and $L$ (resp. $R$ ) is the left (resp. right) translation by $G$. In particular, we obtain an isomorphism of $G$-modules

$$
L^{p}\left(G / K, \mathcal{V}_{\ell}\right) \simeq\left(L^{p}(G) \otimes \wedge^{\ell} \mathfrak{s}\right)^{K},
$$

where $\left(L^{p}(G) \otimes \wedge^{\ell} \mathfrak{s}\right)^{K}$ denotes the subspace of $K$-invariant vectors, equipped with the natural norm

$$
\|\phi\|_{L^{p}\left(G / K, \mathcal{V}_{\ell}\right)}=\left(\int_{G}\|\phi(g)\|_{\Lambda^{\ell_{\mathfrak{S}}}}^{p} d g\right)^{\frac{1}{p}}
$$


When $\ell=0$, i.e in the case of functions, we will simply write $L^{p}(G / K)$. Next the group $K$ acts on the vector space $\operatorname{End}\left(\Lambda^{\ell} \mathfrak{s}\right)$ of complex endomorphisms of $\Lambda^{\ell} \mathfrak{s}$ as follows

$$
\widetilde{\sigma}_{\ell}(k)(T)=\sigma_{\ell}(k) \circ T \circ \sigma_{\ell}(k)^{-1}, \quad \forall k \in K, \forall T \in \operatorname{End}\left(\Lambda^{\ell} \mathfrak{s}\right) .
$$

This representation induces a homogeneous vector bundle $\mathcal{E} n d\left(\Lambda^{\ell} \mathfrak{s}\right)$ over $G / K$ and $L^{p}\left(G / K, \mathcal{E} n d\left(\Lambda^{\ell} \mathfrak{s}\right)\right)$ will denote the space of $L^{p}$-sections. We also have an isomorphism of $G$-modules

$$
L^{p}\left(G / K, \mathcal{E} n d\left(\Lambda^{\ell} \mathfrak{s}\right)\right) \simeq\left(L^{p}(G) \otimes \operatorname{End}\left(\Lambda^{\ell} \mathfrak{s}\right)\right)^{K},
$$

for the $K$-action $R \otimes \widetilde{\sigma}_{\ell}$, with the norm

$$
\|\phi\|_{L^{p}\left(G / K, \mathcal{E} n d\left(\Lambda^{\ell} \mathfrak{s}\right)\right)}=\left(\int_{G}\|\phi(g)\|_{\operatorname{End}\left(\Lambda^{\ell} \mathfrak{s}\right)}^{p} d g\right)^{\frac{1}{p}} .
$$

In the case where $p=+\infty$, these definitions are adapted as usual.

2.4. Laplacian on square integrable differential forms on $G / K$. The Killing form of $\mathfrak{g}$ induces a sequence of $G$-equivariant maps

$$
\operatorname{End}(\mathfrak{g}) \stackrel{\text { canonical }}{\longrightarrow} \mathfrak{g} \otimes \mathfrak{g}^{\star} \stackrel{\text { Killing }}{\longrightarrow} \mathfrak{g} \otimes \mathfrak{g} \stackrel{\text { injection }}{\longrightarrow} T(\mathfrak{g}) \stackrel{\text { quotient }}{\longrightarrow} \mathcal{U}(\mathfrak{g}),
$$

where $\operatorname{End}(\mathfrak{g})$ denotes the vector space of complex endomorphisms of $\mathfrak{g}, \mathfrak{g}^{\star}$ the vector dual of $\mathfrak{g}, T(\mathfrak{g})$ the tensor algebra of $\mathfrak{g}$ and $\mathcal{U}(\mathfrak{g})$ the enveloping algebra of $\mathfrak{g}$. Let $\Omega_{G}$ be the image in (the center of) $\mathcal{U}(\mathfrak{g})$ of the identity. Any element $A=A_{1} \cdot A_{2} \cdots A_{p}$ of $\mathcal{U}(\mathfrak{g})$ defines a differential operator $\tilde{A}$ on $G$

$$
\tilde{A}=\tilde{A}_{1} \circ \cdots \circ \tilde{A}_{p} \text { where }\left(\tilde{A}_{j} f\right)(g)=\left.\frac{d}{d t}\right|_{t=0} f\left(\exp \left(-t A_{j}\right) g\right) \forall f \in C^{\infty}(G), g \in G .
$$

In particular, the $G$-invariant differential operator $\widetilde{\Omega}_{G}$ on $G$ is the Casimir operator of $G$. Similarly we define the Casimir operators $\widetilde{\Omega}_{K}$ of $K$ and $\widetilde{\Omega}_{M_{i}}$ of $M_{i}$. The representation $\pi_{P_{i}, \delta_{i}, \alpha_{i}}$ defines, by differentiation, an action of $\mathcal{U}(\mathfrak{g})$ on the smooth vectors of $\mathcal{H}_{P_{i}, \delta_{i}, \alpha_{i}}$. We will denote this action by the same symbol $\pi_{P_{i}, \delta_{i}, \alpha_{i}}$. It is known that $\widetilde{\Omega}_{G}$ acts as a scalar operator on the (smooth vectors of the) principal series representation $\pi_{P_{i}, \delta_{i}, \alpha_{i}}$ (see Proposition 8.22 of [20])

$$
\pi_{P_{i}, \delta_{i}, \alpha_{i}}\left(\widetilde{\Omega}_{G}\right)=\omega_{\delta_{i}, \alpha_{i}} \mathrm{Id}
$$

with

$$
\omega_{P_{i}, \delta_{i}, \alpha_{i}}=\left\|\operatorname{char}\left(\delta_{i}\right)\right\|^{2}+\left\|\alpha_{i}\right\|^{2}-\left\|\rho_{i}\right\|^{2}=\delta_{i}\left(\widetilde{\Omega}_{M_{i}}\right)+\left\|\alpha_{i}\right\|^{2}-\left\|\rho_{\mathfrak{a}_{i}}\right\|^{2},
$$

where $\operatorname{char}\left(\delta_{i}\right)$ denotes the infinitesimal character of $\delta_{i}$. If we let

$$
Q_{\ell}=\int_{K} R(k) \otimes \sigma_{\ell}(k) d k
$$

be the projection of $L^{2}(G) \otimes \wedge^{\ell} \mathfrak{s}$ onto the subspace $\left(L^{2}(G) \otimes \wedge^{\ell} \mathfrak{s}\right)^{K}$ of $K$-invariant vectors, then the Laplacian $\Delta_{\ell}$ acting on square integrable $\ell$-forms on $G / K$ is defined by

$$
\Delta_{\ell} \circ Q_{\ell}=-Q_{\ell} \circ\left(\widetilde{\Omega}_{G} \otimes \operatorname{Id}_{\wedge \ell_{\mathfrak{s}}}\right) .
$$


On the other hand, the spectrum $\operatorname{sp}\left(\Delta_{\ell}\right)$ of $\Delta_{\ell}$ decomposes as a discrete spectrum $s p_{d}\left(\Delta_{\ell}\right)$ and continuous spectrum $s p_{c}\left(\Delta_{\ell}\right)$

$$
s p\left(\Delta_{\ell}\right)=s p_{c}\left(\Delta_{\ell}\right) \cup s p_{d}\left(\Delta_{\ell}\right) .
$$

It is well known that (see Theorem A and B in [8], and Proposition 1.2 in [33])

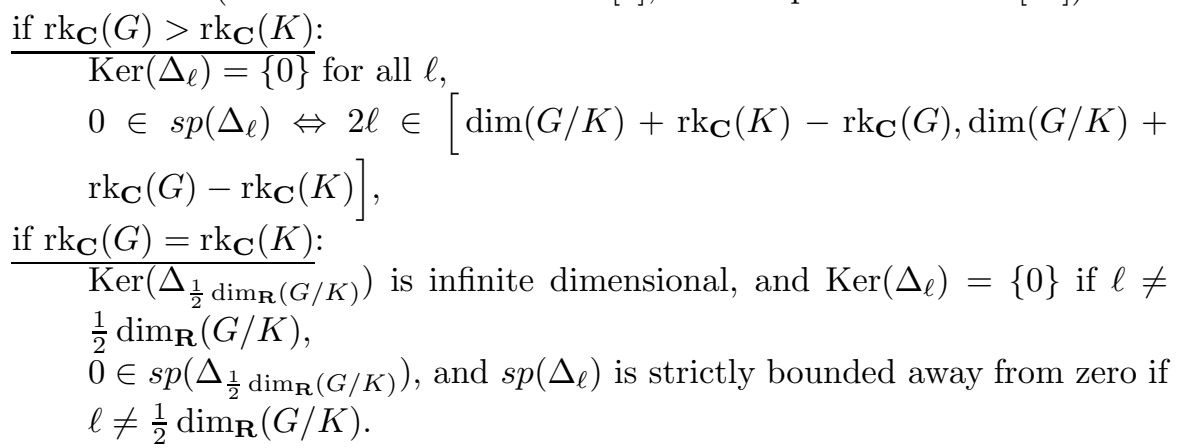

For the harmonic $\ell$-forms, writing

$$
\widehat{G}\left(\sigma_{\ell}\right)=\left\{\pi \in \widehat{G}_{d} \mid \operatorname{char}(\pi)=\operatorname{char}\left(\mathbb{1}_{G}\right)\right\}
$$

where $\mathbb{1}_{G}$ denotes the trivial representation of $G$, we have

$$
\operatorname{Ker}\left(\Delta_{\ell}\right)=\left\{\begin{array}{l}
\sum_{\pi \in \widehat{G}\left(\sigma_{\ell}\right)} \mathcal{H}_{\pi} \text { if } \operatorname{rk}_{\mathbf{C}}(G)=\operatorname{rk}_{\mathbf{C}}(K) \text { and } \ell=\frac{1}{2} \operatorname{dim}_{\mathbf{R}}(G / K) \\
\{0\} \text { otherwise. }
\end{array}\right.
$$

In other words, $\operatorname{Ker}\left(\Delta_{\ell}\right)$ is either reduced to $\{0\}$ or is infinite dimensional. Observe that the number $\operatorname{dim}_{\mathbf{R}}(G / K)+\operatorname{rk}_{\mathbf{C}}(K)-\operatorname{rk}_{\mathbf{C}}(G)$ is positive and $\operatorname{dim}_{\mathbf{R}}(G / K)+\operatorname{rk}_{\mathbf{C}}(G)-\operatorname{rk}_{\mathbf{C}}(K)$ is always even. A throughout discussion on the spectrum of $\Delta_{\ell}$ for more general manifolds is given in [27].

2.5. Plancherel formula for square integrable differential forms on $G / K$. Following (2.6) the space of square integrable $\ell$-forms decomposes under the action of $G$ into a continuous part and a discrete part

$$
L^{2}\left(G / K, \mathcal{V}_{\ell}\right)=L^{2}\left(G / K, \mathcal{V}_{\ell}\right)_{c} \oplus L^{2}\left(G / K, \mathcal{V}_{\ell}\right)_{d}
$$

The Harish-Chandra Plancherel formula decomposes the continuous part of the biregular representation $L \otimes R$ of $G$ as

$$
L^{2}(G)_{c} \simeq \sum_{i, \operatorname{dim}\left(\mathfrak{a}_{i}\right)>0} \sum_{\delta_{i} \in\left(\widehat{M}_{i}\right)_{d}} \int_{\mathfrak{a}_{i, 0}^{\star}}^{\widehat{\oplus}} \mathcal{H}_{\delta_{i}, \sqrt{-1} \nu_{i}} \widehat{\otimes} \mathcal{H}_{P_{i}, \delta_{i}, \sqrt{-1} \nu_{i}}^{\star} \mathbf{c}_{\delta_{i}}\left(\sqrt{-1} \nu_{i}\right) d \nu_{i},
$$

where $\widehat{\oplus}$ (resp. $\widehat{\otimes}$ ) denotes the Hilbert sum (resp. product), $d \nu_{i}$ a Lebesgue measure on $\mathfrak{a}_{i, 0}^{\star}$ and, for a fixed $\delta_{i} \in\left(\widehat{M}_{i}\right)_{d}$, the function $\mathbf{c}_{\delta_{i}}$ is the Plancherel density. It is known that $\mathbf{c}_{\delta_{i}}$ is a non-negative continuous function with polynomial growth on $\mathfrak{a}_{i, 0}^{\star}$ (Theorem 19 of [18]), i.e there exist a positive real number $b_{i}$ and a non negative integer $N_{i} \in 4 \mathbf{N}$ (both depending on $\delta_{i}$ ) such that

$$
\mathbf{c}_{\delta_{i}}\left(\sqrt{-1} \nu_{i}\right) \leq b_{i}\left(1+\left\|\nu_{i}\right\|^{2}\right)^{\frac{N_{i}}{4}} .
$$


We have

$$
L^{2}(G)_{c} \otimes \Lambda^{\ell} \mathfrak{s} \simeq \sum_{i, \operatorname{dim}\left(\mathfrak{a}_{i}\right)>0} \sum_{\delta_{i} \in\left(\widehat{M}_{i}\right)_{d}} \int_{\mathfrak{a}_{i, 0}^{\star}}^{\widehat{\oplus}} \mathcal{H}_{\delta_{i}, \sqrt{-1}} \nu_{i} \widehat{\otimes}\left(\mathcal{H}_{P_{i}, \delta_{i}, \sqrt{-1} \nu_{i}}^{\star} \otimes \Lambda^{\ell} \mathfrak{s}\right) \mathbf{c}_{\delta_{i}}\left(\sqrt{-1} \nu_{i}\right) d \nu_{i}
$$

so that

$$
\begin{aligned}
&\left(L^{2}(G)_{c} \otimes \Lambda^{\ell} \mathfrak{s}\right)^{K} \\
&(2.9) \simeq \sum_{i, \operatorname{dim}\left(\mathfrak{a}_{i}\right)>0} \sum_{\delta_{i} \in\left(\widehat{M}_{i}\right)_{d}} \int_{\mathfrak{a}_{i, 0}^{\star}}^{\widehat{\oplus}} \mathcal{H}_{\delta_{i}, \sqrt{-1} \nu_{i}} \widehat{\otimes}\left(\mathcal{H}_{P_{i}, \delta_{i}, \sqrt{-1} \nu_{i}}^{\star} \otimes \Lambda^{\ell} \mathfrak{s}\right)^{K} \mathbf{c}_{\delta_{i}}\left(\sqrt{-1} \nu_{i}\right) d \nu_{i} .
\end{aligned}
$$

Next the Frobenius reciprocity Theorem implies that the restriction $\operatorname{Ind}_{M_{i} \cap K}^{K}\left(\left.\delta_{i}\right|_{M_{i} \cap K}\right.$ ) of $\pi_{\delta_{i}, \sqrt{-1} \nu_{i}}$ to $K$ does not depend on $\nu_{i}$. If we let $\mathcal{H}_{\delta_{i}}$ be the completion of the complex vector space $C^{\infty}\left(K ; \delta_{i}\right)$ with respect to the norm given by

$$
\|f\|_{\mathcal{H}_{\delta_{i}}}=\left(\int_{K}\|f(k)\|_{V_{\delta_{i}}}^{2} d k\right)^{1 / 2}
$$

then the restriction to $K$ is an isometry of $\mathcal{H}_{P_{i}, \delta_{i}, \sqrt{-1} \nu_{i}}$ onto the $K$-module $\mathcal{H}_{\delta_{i}}$. Moreover the complex vector space $\left(\mathcal{H}_{\delta_{i}, \sqrt{-1} \nu_{i}}^{*} \otimes \Lambda^{\ell} \mathfrak{s}\right)^{K}$ is isomorphic, as a $K$-module, to the space $\operatorname{Hom}_{K}\left(\mathcal{H}_{\delta_{i}}, \Lambda^{\ell} \mathfrak{s}\right)$ of $K$-equivariant homomorphisms from $\mathcal{H}_{\delta_{i}}$ onto $\mathcal{V}_{l}$, so that (2.9) becomes

$$
L^{2}\left(G / K, \mathcal{V}_{\ell}\right)_{c} \simeq \sum_{i, \operatorname{dim}\left(\mathfrak{a}_{i}\right)>0} \sum_{\delta_{i} \in\left(\widehat{M}_{i}\right)_{d}} \int_{\mathfrak{a}_{i, 0}^{\star}}^{\widehat{\oplus}} \mathcal{H}_{\delta_{i}, \sqrt{-1} \nu_{i}} \widehat{\otimes} \operatorname{Hom}_{K}\left(\mathcal{H}_{\delta_{i}}, \Lambda^{\ell} \mathfrak{s}^{\star}\right) \mathbf{c}_{\delta_{i}}\left(\sqrt{-1} \nu_{i}\right) d \nu_{i}
$$

In particular the only principal series representations $\pi_{P_{i}, \delta_{i}, \sqrt{-1} \nu_{i}}$ of $G$ appearing in the above decomposition are those satisfying $\operatorname{Hom}_{M_{i} \cap K}\left(\sigma_{\ell}, \delta_{i}\right) \neq\{0\}$. We deduce that (see Section 5 of $[26]$ )

$$
\begin{aligned}
& L^{2}\left(G / K, \mathcal{V}_{\ell}\right)_{c} \\
& \simeq \sum_{i, \operatorname{dim}\left(\mathfrak{a}_{i}\right)>0} \sum_{\delta_{i} \in \widehat{M}_{i}\left(\sigma_{\ell}\right)} \int_{\mathfrak{a}_{i, 0}^{\star}}^{\widehat{\oplus}} \mathcal{H}_{P_{i}, \delta_{i}, \sqrt{-1} \nu_{i}} \widehat{\otimes}\left(\mathcal{H}_{P_{i}, \delta_{i}, \sqrt{-1} \nu_{i}}^{\star} \otimes \Lambda^{\ell} \mathfrak{s}\right)^{K} \mathbf{c}_{\delta_{i}}\left(\sqrt{-1} \nu_{i}\right) d \nu_{i},
\end{aligned}
$$

where

$$
\widehat{M}_{i}\left(\sigma_{\ell}\right) \stackrel{\text { def. }}{=}\left\{\delta \in\left(\widehat{M}_{i}\right)_{d} \mid \operatorname{Hom}_{M_{i} \cap K}\left(\sigma_{\ell}, \delta\right) \neq\{0\}\right\} .
$$

Similarly for the discrete part, we deduce, from (2.7), that

$$
L^{2}\left(G / K, \mathcal{V}_{\ell}\right)_{d}=\left\{\begin{array}{l}
\sum_{\pi \in \widehat{G}\left(\sigma_{\ell}\right)} \mathcal{H}_{\pi} \otimes \operatorname{Hom}_{K}\left(\mathcal{H}_{\pi}, \Lambda^{\ell_{\mathfrak{S}^{\star}}}\right) \text { if } \operatorname{rk}_{\mathbf{C}}(G)=\operatorname{rk}_{\mathbf{C}}(K) \\
\text { and } \ell=\frac{1}{2} \operatorname{dim}_{\mathbf{R}}(G / K), \\
\{0\} \text { otherwise. }
\end{array}\right.
$$


Therefore the Plancherel formula for square integrable $\ell$-forms is given by

$$
\begin{aligned}
& \quad L^{2}\left(G / K, \mathcal{V}_{\ell}\right) \\
& \simeq \sum_{i, \operatorname{dim}\left(\mathfrak{a}_{i}\right)>0} \sum_{\delta_{i} \in \widehat{M}_{i}\left(\sigma_{\ell}\right)} \int_{\mathfrak{a}_{i, 0}^{\star}}^{\widehat{\oplus}} \mathcal{H}_{P_{i}, \delta_{i}, \sqrt{-1} \nu_{i}} \widehat{\otimes}\left(\mathcal{H}_{P_{i}, \delta_{i}, \sqrt{-1} \nu_{i}}^{\star} \otimes \Lambda^{\ell} \mathfrak{s}\right)^{K} \mathbf{c}_{\delta_{i}}\left(\sqrt{-1} \nu_{i}\right) d \nu_{i} \\
& +\sum_{\pi \in \widehat{G}\left(\sigma_{\ell}\right)} \mathcal{H}_{\pi} \otimes \operatorname{Hom}_{K}\left(\mathcal{H}_{\pi}, \Lambda^{\ell} \mathfrak{s}^{\star}\right) \\
& \quad \text { if } \operatorname{rk}_{\mathbf{C}}(G)=\operatorname{rk}_{\mathbf{C}}(K) \text { and } \ell=\frac{1}{2} \operatorname{dim}_{\mathbf{R}}(G / K), \\
& \quad L^{2}\left(G / K, \mathcal{V}_{\ell}\right) \\
& \quad \sum_{i, \operatorname{dim}\left(\mathfrak{a}_{i}\right)>0} \sum_{\delta_{i} \in \widehat{M}_{i}\left(\sigma_{\ell}\right)} \int_{\mathfrak{a}_{i, 0}^{\star}}^{\widehat{\oplus}} \mathcal{H}_{P_{i}, \delta_{i}, \sqrt{-1} \nu_{i}} \widehat{\otimes}\left(\mathcal{H}_{P_{i}, \delta_{i}, \sqrt{-1} \nu_{i}}^{\star} \otimes \Lambda^{\ell} \mathfrak{s}\right)^{K} \mathbf{c}_{\delta_{i}}\left(\sqrt{-1} \nu_{i}\right) d \nu_{i} \\
& \quad \text { if } \operatorname{rk}_{\mathbf{C}}(G) \neq \operatorname{rk}_{\mathbf{C}}(K) \text { or } \ell \neq \frac{1}{2} \operatorname{dim}_{\mathbf{R}}(G / K) .
\end{aligned}
$$

2.6. Spherical Fourier transform and inverse Fourier transform. Consider the decomposition (2.3) of the isotropy representation and write $\operatorname{pr}_{j}$ for the corresponding projection

$$
\operatorname{pr}_{j}: V_{\ell} \rightarrow V_{\ell}^{j}
$$

Let $f: G \rightarrow \Lambda^{\ell} \mathfrak{s}$ be a compactly supported smooth map which is $K$-equivariant, i.e

$$
f(g k)=\sigma_{\ell}^{-1}(k) f(g), \quad \forall g \in G, k \in K .
$$

We decompose $f$ as the sum

$$
f=\sum_{j} f^{j}
$$

of $K$-equivariant maps, where

$$
f^{j}=\operatorname{pr}_{j} \circ f .
$$

Similarly to (2.10), define for each $j$ the set

$$
\widehat{M}_{i}\left(\sigma_{\ell}^{j}\right) \stackrel{\text { def. }}{=}\left\{\delta \in\left(\widehat{M}_{i}\right)_{d} \mid \operatorname{Hom}_{M_{i} \cap K}\left(\sigma_{\ell}^{j}, \delta\right) \neq\{0\}\right\} .
$$

For $\delta_{i}, j$ and $\ell$ fixed, let $\left\{T_{\delta_{i}, r}^{\ell, j}\right\}_{r \geq 1}$ be an orthonormal basis of the (finite dimensional) complex vector space $\operatorname{Hom}_{K}\left(\mathcal{H}_{\delta_{i}}, V_{\ell}^{j}\right)$ with respect to the usual scalar product

$$
\langle B, C\rangle=\frac{1}{\operatorname{dim}\left(V_{\ell}^{j}\right)} \operatorname{Tr}\left(B^{\star} C\right)
$$

where $B^{\star}$ denotes the adjoint of $B$. Define the maps

$$
T_{\delta_{i}}^{\ell, j}=\sum_{r} T_{\delta_{i}, r}^{\ell, j} \text { and } T_{\delta_{i}}^{\ell, j \star}=\sum_{r} T_{\delta_{i}, r}^{\ell, j \star} .
$$


Now the Fourier transform $\widehat{f^{j}}$ of $f^{j}$ is the map

$$
\widehat{f^{j}}: \widehat{M}_{i}\left(\sigma_{\ell}^{j}\right) \times \sqrt{-1} \mathfrak{a}_{i, 0}^{\star} \rightarrow \mathcal{H}_{P_{i}, \delta_{i}, \sqrt{-1} \nu_{i}} \times \operatorname{Hom}_{K}\left(\mathcal{H}_{\delta_{i}}, V_{\ell}^{j}\right)
$$

defined by

$$
\widehat{f^{j}}\left(\delta_{i}, \sqrt{-1} \nu_{i}\right)=\frac{1}{\operatorname{dim}\left(V_{\ell}^{j}\right)} \int_{G} \pi_{P_{i}, \delta_{i}, \sqrt{-1} \nu_{i}}(g) \circ T_{\delta_{i}}^{\ell, j \star}\left(f^{j}(g)\right) \otimes T_{\delta_{i}}^{\ell, j} d g .
$$

The inverse Fourier transform is given by

$$
\begin{aligned}
& f^{j}(g) \\
& =\sum_{i} \sum_{\delta_{i} \in \widehat{M_{i}}\left(\sigma_{\ell}^{j}\right)} \frac{1}{\left|W_{i}\right|} \frac{1}{\operatorname{dim}\left(V_{\ell}^{j}\right)} \int_{\mathfrak{a}_{i, 0}^{\star}} \Phi_{\delta_{i}}^{j}\left(\pi_{P_{i}, \delta_{i}, \sqrt{-1} \nu_{i}}\left(g^{-1}\right) \otimes 1_{\operatorname{Hom}_{K}\left(\mathcal{H}_{\delta_{i}}, V_{\ell}^{j}\right)}\left(\widehat{f^{j}}\left(\delta_{i}, \sqrt{-1} \nu_{i}\right)\right)\right) \\
& \times \mathbf{c}_{\delta_{i}}\left(\sqrt{-1} \nu_{i}\right) d \nu_{i}
\end{aligned}
$$

where

$$
\Phi_{\delta_{i}}^{j}: \mathcal{H}_{P_{i}, \delta_{i}, \sqrt{-1} \nu_{i}} \otimes \operatorname{Hom}_{K}\left(\mathcal{H}_{\delta_{i}}, V_{\ell}^{j}\right) \rightarrow V_{\ell}^{j}
$$

is the contraction map. The Fourier transform of $f$ is $\widehat{f}=\sum_{j} \widehat{f^{j}}$. It should be noted that when $G$ has a non empty discrete series, then $P_{i}=G$ for some $i$, with $A_{i}=N_{i}=\{e\}$ and $\mathbf{c}_{\delta_{i}}(0)>0$.

REMARK 2.14. When $\ell=0, f=f^{j}: G \rightarrow \mathbf{C}$ is a compactly supported complexvalued function on $G$. In this case, one has

$$
\begin{aligned}
& \left.\Phi_{\delta_{i}}^{j}\left(\pi_{P_{i}, \delta_{i}, \sqrt{-1} \nu_{i}}\left(g^{-1}\right) \otimes 1_{\text {Hom }_{K}\left(\mathcal{H}_{\delta_{i}}, V_{\ell}^{j}\right)} \widehat{\left(f^{j}\right.}\left(\delta_{i}, \sqrt{-1} \nu_{i}\right)\right)\right) \\
& =\operatorname{Tr}\left(\pi_{P_{i}, \delta_{i}, \sqrt{-1} \nu_{i}}\left(g^{-1}\right) \circ \widehat{f^{j}}\left(\delta_{i}, \sqrt{-1} \nu_{i}\right)\right)
\end{aligned}
$$

so that our formulas (2.12) and (2.13) reduce to

$$
\widehat{f}\left(\delta_{i}, \sqrt{-1} \nu_{i}\right)=\int_{G} \pi_{P_{i}, \delta_{i}, \sqrt{-1} \nu_{i}}(g) f(g) d g
$$

and

$$
f(g)=\sum_{i} \sum_{\delta_{i} \in\left(\widehat{M}_{i}\right)_{d}} \frac{1}{\left|W_{i}\right|} \int_{\mathfrak{a}_{i, 0}^{\star}} \operatorname{Tr}\left(\pi_{P_{i}, \delta_{i}, \sqrt{-1} \nu_{i}}\left(g^{-1}\right) \circ \widehat{f}\left(\delta_{i}, \sqrt{-1} \nu_{i}\right)\right) \mathbf{c}_{\delta_{i}}\left(\sqrt{-1} \nu_{i}\right) d \nu_{i}
$$

which are respectively the Harish-Chandra Fourier transform and inverse Fourier transform for complex-valued functions on $G$ [18].

2.7. Spherical functions on $G$. In the sequel, it will be useful to write the Fourier transform in term of some spherical functions on $G$. From (2.12) we have

$$
\begin{aligned}
& \left(\pi_{P_{i}, \delta_{i}, \sqrt{-1} \nu_{i}}\left(g^{-1}\right) \otimes 1_{\operatorname{Hom}_{K}\left(\mathcal{H}_{\delta_{i}}, V_{\ell}^{j}\right)}\left(\widehat{f^{j}}\left(\delta_{i}, \sqrt{-1} \nu_{i}\right)\right)\right) \\
& =\frac{1}{\operatorname{dim}\left(V_{\ell}^{j}\right)} \int_{G} \pi_{P_{i}, \delta_{i}, \sqrt{-1} \nu_{i}}\left(g^{\prime}\right) T_{\delta_{i}}^{\ell, j \star}\left(f^{j}\left(g g^{\prime}\right)\right) \otimes T_{\delta_{i}}^{\ell, j} d g^{\prime}
\end{aligned}
$$


so that

$$
\begin{aligned}
& \Phi_{\delta_{i}}^{j}\left(\pi_{P_{i}, \delta_{i}, \sqrt{-1} \nu_{i}}\left(g^{-1}\right) \otimes 1_{\operatorname{Hom}_{K}\left(\mathcal{H}_{\delta_{i}}, V_{\ell}^{j}\right)}\left(\widehat{f^{j}}\left(\delta_{i}, \sqrt{-1} \nu_{i}\right)\right)\right) \\
= & \frac{1}{\operatorname{dim}\left(V_{\ell}^{j}\right)} \int_{G} \Psi_{\delta_{i}, \sqrt{-1} \nu_{i}}^{\ell, j}\left(g^{\prime}\right)\left(f^{j}\left(g g^{\prime}\right)\right) d g^{\prime} \\
= & \frac{1}{\operatorname{dim}\left(V_{\ell}^{j}\right)}\left(\Psi_{\delta_{i}, \sqrt{-1} \nu_{i}}^{\ell, j} \star f^{j}\right)(g),
\end{aligned}
$$

where $\Psi_{\delta_{i}, \sqrt{-1} \nu_{i}}^{\ell, j}$ is the $\operatorname{End}\left(V_{\ell}^{j}\right)$-valued function on $G$ defined by

$$
\Psi_{\delta_{i}, \nu_{i}}^{\ell, j}(g)=T_{\delta_{i}}^{\ell, j} \circ \pi_{P_{i}, \delta_{i}, \sqrt{-1} \nu_{i}}(g) \circ T_{\delta_{i}}^{\ell, j \star} \forall g \in G .
$$

$\Psi_{\delta_{i}, \nu_{i}}^{\ell, j}$ is an $\operatorname{End}\left(V_{\ell}^{j}\right)$-valued $\sigma_{\ell}^{j}$-spherical function on $G$ satisfying the following property $[11]$

$$
\widetilde{\Omega}_{G} \Psi_{\delta_{i}, \nu_{i}}^{\ell, j}=\omega_{P_{i}, \delta_{i}, \sqrt{-1} \nu_{i}} \Psi_{\delta_{i}, \nu_{i}}^{\ell, j}
$$

REMARK 2.16. If $F$ is an End $\left(V_{l}^{j}\right)$-valued function on $G$, we define the Fourier transform of $F$ as the map

$$
\begin{aligned}
& \widehat{M}_{i}\left(\sigma_{\ell}^{j}\right) \times \sqrt{-1} \mathfrak{a}_{i, 0}^{\star} \rightarrow \operatorname{End}\left(V_{\ell}^{j}\right) \\
&\left(\delta_{i}, \sqrt{-1} \nu_{i}\right) \quad \mapsto \widehat{F}\left(\delta_{i}, \sqrt{-1} \nu_{i}\right)=\int_{G} \Psi_{\delta_{i}, \sqrt{-1} \nu_{i}}^{\ell, j}(g) \circ F(g) d g .
\end{aligned}
$$

The Harish-Chandra spherical function $\Phi_{\lambda}$ on $G$ associated with $\lambda \in \mathfrak{a}^{\star}$ is the function defined by (see Chapter VII of [20])

$$
\Phi_{\lambda}(g)=\int_{K} e^{-\left(\lambda+\rho_{a_{0}}\right)\left(\log \left(a\left(g^{-1} k\right)\right)\right)} d k .
$$

By Proposition 7.4 of [20], for all $K$-finite vectors $u, v$ in $\mathcal{H}_{\delta_{i}}$ there exists a positive real number $d_{i}$ such that for all $g \in G$

$$
\left|\left\langle\pi_{P_{i}, \delta_{i}, \sqrt{-1} \nu_{i}}(g) u, v\right\rangle_{\mathcal{H}_{P_{i}, \delta_{i}, \sqrt{-1} \nu_{i}}}\right| \leq d_{i} \Phi_{0}(g)\|u\|_{\mathcal{H}_{\delta_{i}}}|| v \|_{\mathcal{H}_{\delta_{i}}} .
$$

Moreover the following estimate of the spherical function $\Phi_{0}$ will be useful (see Proposition 2.2.12 in [3]). There exists a positive number $C$ such that, for all $g \in G$

$$
\Phi_{0}(g) \leq C\left(\Pi_{\alpha \in \Sigma^{++}}\left(1+\alpha\left(a^{+}(g)\right)\right)\right) e^{-\rho_{\mathrm{a}_{0}}\left(a^{+}(g)\right)} .
$$

2.8. On Delorme's Paley-Wiener Theorem. We recall a recent result of P. Delorme on the Paley-Wiener theorem of Arthur [16]. We first start with the notion of successive partial derivatives of principal series representations of $G$ introduced by Delorme. For this we will follow the description of van den Ban and Souaifi given in [5]. Let $V$ be a Fréchet space and $\mathcal{V}_{0}$ be a finite dimensional real vector space with complexification $\mathcal{V}$. For any $\eta \in \mathcal{V}^{\star}$ and any holomorphic map $\Phi: \mathcal{V}^{\star} \longrightarrow \operatorname{End}(V)$, one defines the derivative $\Phi^{(\eta)}$ of $\Phi$ along $\eta$ as the following holomorphic map

$$
\frac{\partial}{\partial \eta} \Phi \stackrel{\text { def. }}{=} \Phi^{(\eta)}: \mathcal{V}^{\star} \stackrel{\text { holo. }}{\longrightarrow} \operatorname{End}(V \oplus V)
$$


with

$$
\Phi^{(\eta)}(\lambda)\left(v_{1}, v_{2}\right)=\left(\phi(\lambda) v_{1}+\left.\frac{d}{d z}\left(\phi(\lambda+z \eta) v_{2}\right)\right|_{z=0}, \phi(\lambda) v_{2}\right) .
$$

By iteration, for any finite sequence $\eta=\left(\eta_{1}, \eta_{2}, \cdots, \eta_{N}\right) \in \mathcal{V}^{\star}$, one defines the successive derivative $\Phi^{(\eta)}$ of $\Phi$ along $\eta$ as the map

$$
\Phi^{(\eta)}: \mathcal{V}^{\star} \stackrel{\text { holo. }}{\longrightarrow} \operatorname{End}\left(V^{(\eta)}\right)
$$

with

$$
\Phi^{(\eta)}=\left(\cdots\left(\Phi^{\left(\eta_{N}\right)}\right)^{\left(\eta_{N-1}\right)} \cdots\right)^{\left(\eta_{1}\right)}
$$

where $V^{(\eta)}$ is the direct sum of $2^{N}$ copies of $V$.

Recall, from section 2.1, that $\mathfrak{a}_{0}$ is a maximal abelian subspace in $\mathfrak{s}_{0}$ and $A=$ $\exp \left(\mathfrak{a}_{0}\right)$ is the analytic subgroup of $G$ with Lie algebra $\mathfrak{a}_{0}$. Denote by $\mathcal{P}(A)$ the set of cuspidal parabolic subgroups of $G$ containing $A$. The set $\mathcal{P}(A)$ is finite and each element $P$ of $\mathcal{P}(A)$ has Langlands decomposition $P=M_{P} A_{P} N_{P}$ where $M_{P}$ is reductive, $A_{P}$ abelian and $N_{P}$ nilpotent with Lie algebras $\mathfrak{m}_{P, 0}, \mathfrak{a}_{P, 0}$ and $\mathfrak{n}_{P, 0}$ respectively. Recall that $\rho_{\mathfrak{a}_{P, 0}}$ is the half sum of positive roots in $\mathfrak{g}_{0}$ relative to $\mathfrak{a}_{P, 0}$ counted with their multiplicities, for some fixed positive system for $\mathfrak{a}_{P, 0}$-roots in $\mathfrak{g}_{0}$. Recall that we drop the subscript 0 for the complexification of real vector spaces. We shall write $P_{0}$ for the (standard) minimal parabolic subgroup. Given $\delta \in\left(\widehat{M_{P}}\right)_{d}$, write $\operatorname{End}\left(C^{\infty}(K, \delta)\right)$ for the vector space of endomorphisms of $C^{\infty}(K, \delta)$ and define the map

$$
\pi_{P, \delta,(\cdot)}: G \longrightarrow\left(\mathfrak{a}_{P}^{\star} \longrightarrow \operatorname{End}\left(C^{\infty}(K, \delta)\right)\right), \quad g \mapsto\left(\lambda \mapsto \pi_{P, \delta, \lambda}(g)\right)
$$

where $\pi_{P, \delta, \lambda}$ denotes the principal series representation of $G$ associated with $P, \delta$ and $\lambda$. Here we use the realization of the principal series in the compact picture described in (2.2). Let $\mathcal{D}$ for the set of 4 -tuples $\xi=(P, \delta, \lambda, \eta)$, where $P \in \mathcal{P}(A), \delta \in\left(\widehat{M_{P}}\right)_{d}$, $\lambda \in \mathfrak{a}_{P}^{\star}$ and $\eta$ is a finite sequence in $\mathfrak{a}_{P}^{\star}$. We shall simply write $\mathcal{D}_{P_{0}}$ for the set of 4-tuples $\xi=(P, \delta, \lambda, \eta)$ with $P=P_{0}$. Given $\xi=(P, \delta, \lambda, \eta) \in \mathcal{D}$, we define the partial derivative along $\eta$ of the principal series representation $\pi_{P, \delta, \lambda}$ as the $G$-representation defined by the following map

$$
\pi_{\xi}: G \rightarrow \operatorname{End}\left(C^{\infty}(K, \delta)^{(\eta)}\right), g \mapsto \pi_{P, \delta,(\cdot)}(g)^{(\eta)}(\lambda)
$$

where we have used the notation in (2.20) and (2.21) with $\mathcal{V}_{0}=\mathfrak{a}_{P, 0}$ and $V=$ $C^{\infty}(K, \delta)$. Let $\mathcal{C}\left(\mathfrak{a}_{P}^{\star}\right)$ be the vector space of complex functions on $\mathfrak{a}_{P}^{\star}$ and $\mathcal{O}\left(\mathfrak{a}_{P}^{\star}\right)$ the vector space of complex valued holomorphic functions on $\mathfrak{a}_{P}^{\star}$. Write $\mathcal{S}(P ; \delta)$ for the space of bi- $K$-finite elements of $\operatorname{End}\left(C^{\infty}(K, \delta)\right)$. Then, for an element

$$
\phi \in \bigoplus_{P \in \mathcal{P}(A)} \bigoplus_{\delta \in\left(\widehat{M_{P}}\right)_{d}}\left(\mathcal{O}\left(\mathfrak{a}_{P}^{\star}\right) \otimes \mathcal{S}(P ; \delta)\right),
$$

we define in a similar way $\phi_{\xi} \in \operatorname{End}\left(C^{\infty}(K, \delta)^{(\eta)}\right)$. Given a finite sequence $\xi=$ $\left(\xi_{1}, \xi_{2}, \cdots, \xi_{N}\right)$ of elements in $\mathcal{D}$, we define

$$
\pi_{\xi}=\pi_{\xi_{1}} \oplus \pi_{\xi_{2}} \oplus \cdots \oplus \pi_{\xi_{N}} \text { and } \phi_{\xi}=\phi_{\xi_{1}} \oplus \phi_{\xi_{2}} \oplus \cdots \oplus \phi_{\xi_{N}} .
$$

We can now state Delorme's intertwining conditions for a map $\phi$ as in (2.23). 
(i) For each finite sequence $\xi \in \mathcal{D}^{N}$, the map $\phi_{\xi}$ preserves all invariant subspaces of $\pi_{\xi}$, and

(ii) for any two finite sequences $\xi^{1} \in \mathcal{D}^{N_{1}}$ and $\xi^{2} \in \mathcal{D}^{N_{2}}$, and any two sequences of closed invariant subspaces $U_{j} \subset V_{j}$ for $\pi_{\xi^{j}}$, the induced maps $\widetilde{\phi}_{\xi^{j}} \in \operatorname{End}\left(V_{j} / U_{j}\right)$ are intertwined by all intertwining operators $T: V_{1} / U_{1} \longrightarrow$ $V_{2} / U_{2}$.

There is a third condition. In the compact picture realization of principal series representations (2.2), each compactly supported smooth function $f \in C_{c}^{\infty}(G)$ has an operator valued Fourier transform defined by (see (2.15))

$$
\widehat{f}(P, \delta, \lambda) \stackrel{\text { def. }}{=} \pi_{P, \delta, \lambda}(f)=\int_{G} f(g) \pi_{P, \delta, \lambda}(g) d g \in \operatorname{End}\left(C^{\infty}(K ; \delta)\right) .
$$

In particular, if $f$ is bi- $K$-finite then $\widehat{f}(P, \delta, \lambda)$ belongs to $\mathcal{S}(P ; \delta)$. Define the prePaley-Wiener space $P W_{P}^{\text {pre }}(G, K, r)$ associated with the parabolic $P \in \mathcal{P}(A)$ as the space of maps $\phi \in \bigoplus_{\delta \in\left(\widehat{M_{P}}\right)_{d}} \mathcal{O}\left(\mathfrak{a}_{P}^{\star}\right) \otimes \mathcal{S}(P ; \delta)$ for which there exists a number $r>0$ and for every $n>0$ a number $C_{n}>0$ such that

(iii) $)_{r}\|\widehat{\phi}(P, \delta, \lambda)\| \leq C_{n}(1+|\lambda|)^{-n} e^{r|\operatorname{Re}(\lambda)|}$ for all $\delta$ and $\lambda$, where $\operatorname{Re}(\lambda)$ denotes the real part of $\lambda$. Then Delorme's Paley-Wiener space is the vector space defined by (Définition 3 in [16])

$$
\begin{gathered}
\mathcal{P} \mathcal{W}_{r}(G, K)=\left\{\phi \in \oplus_{P \in \mathcal{P}(A)} \oplus_{\delta \in\left(\widehat{M_{P}}\right)_{d}} \mathcal{O}\left(\mathfrak{a}_{P}^{\star}\right) \otimes \mathcal{S}(P ; \delta) \mid \phi\right. \text { statisfies } \\
\text { conditions } \left.(i),(i i) \text { and }(i i i)_{r}\right\} .
\end{gathered}
$$

Finally, we can now state the Paley-Wiener theorem proved by Delorme.

Paley-Wiener Theorem (Théorème 2 of [16]). Let $D_{r}(G)$ be the space of complexvalued functions on $G$ which are compactly supported in the closed ball in $G$ of radius $r$ and center the neutral element of $G$. The map $D_{r}(G) \rightarrow \mathcal{P} \mathcal{W}_{r}(G, K), f \mapsto \widehat{f}$ is a topological isomorphism of Fréchet spaces.

Unfortunately Delorme's intertwining conditions, especially condition (ii), are not easy to check, even in particular cases. It turns out that, using recent results of van den Ban and Souaifi (Lemmas 4.1 and 4.2 in [5]), one can reduce considerably these intertwining conditions enabling us to use Delorme's Paley-Wiener theorem in our specific situation. Since we shall make an essential use of van den Ban and Souaifi's observation, and for the convenience of the reader, we include the proof of this reduction.

Proposition 2.26. [5] Let $\phi$ be a map as in (2.23). Then one has

(1) the map $\phi$ satisfies Delorme's intertwining conditions if, and only if, it statisfies condition (i).

(2) The following assertions are equivalent

(a) $\phi$ satisfies (i) for each finite sequence of data in $\mathcal{D}$.

(b) $\phi$ satisfies (i) for each finite sequence of data in $\mathcal{D}_{P_{0}}$.

Proof. For (1), let $\xi^{j}, \pi_{\xi^{j}}, U_{j}$ and $V_{j}$ be as in (ii), for $j=1,2$. Let $T: V_{1} / U_{1} \longrightarrow$ $V_{2} / U_{2}$ be an intertwining operator. In particular $T$ is equivariant and the graph of $T$ is an invariant subspace of $V_{1} / U_{1} \oplus V_{2} / U_{2}$. Since $\phi$ statisfies (i), the map $\widetilde{\phi}_{\xi^{1}} \oplus \widetilde{\phi}_{\xi^{2}}$ preserves the graph of $T$, i.e $T \circ \widetilde{\phi}_{\xi^{1}}=\widetilde{\phi}_{\xi^{2}} \circ T$.

For $(2),(a) \Rightarrow(b)$ is obvious. For the other direction, one proceeds in several steps. 
- Fix a 4-tuple $\xi=\left(P, \delta, \lambda_{0}, \eta\right) \in \mathcal{D}$, where $P \in \mathcal{P}(A)$ is a cuspidal parabolic subgroup of $G$ containing $A$ with Langlands decomposition $P=M_{P} A_{P} N_{P}$. The group $M_{P}$ is a real reductive subgroup of $G$ with Cartan decomposition $M_{P}=\left(M_{P} \cap\right.$ $K) \exp \left(\mathfrak{m}_{P, 0} \cap \mathfrak{s}_{0}\right)$. Let $\mathfrak{a}_{P, 0}^{\prime}$ be a maximal abelian subspace of $\mathfrak{m}_{P, 0} \cap \mathfrak{s}_{0}$ so that

$$
\mathfrak{a}_{0}=\mathfrak{a}_{P, 0} \oplus \mathfrak{a}_{P, 0}^{\prime} .
$$

- By the subrepresentation theorem, there exist a (minimal) parabolic subgroup $Q_{P}^{\prime}$ of $M_{P}$ with Langlands decomposition

$$
Q_{P}^{\prime}=M_{P}^{\prime} A_{P}^{\prime} N_{P}^{\prime}
$$

where $A_{P}^{\prime}=\exp \left(\mathfrak{a}_{P, 0}^{\prime}\right)$, a unitary irreducible representation $\sigma \in \widehat{M_{P}^{\prime}}$ of $M_{P}^{\prime}$ and linear form $\mu \in \mathfrak{a}_{P}^{\prime \star}$ on $\mathfrak{a}_{P}^{\prime}$ such that

$$
\delta \simeq \text { subrepresentation of } \operatorname{Ind}_{Q_{P}^{\prime}}^{M_{P}} \sigma \otimes \mu \otimes 1 .
$$

- There exists a minimal parabolic subgroup $Q_{P}$ of $G$ containing $A$ such that $Q_{P} \in \mathcal{P}(A)$ and

$$
Q_{P} \cap M_{P}=Q_{P}^{\prime}
$$

Then, using induction by stages, one obtains that:

$$
\begin{aligned}
\operatorname{Ind}_{P}^{G} \delta \otimes \lambda_{0} \otimes 1 & \simeq \text { subrepresentation of } \operatorname{Ind}_{P}^{G}\left(\operatorname{Ind}_{Q_{P}^{\prime}}^{M_{P}} \sigma \otimes \mu \otimes 1\right) \otimes \lambda_{0} \otimes 1 \\
& \simeq \text { subrepresentation of } \operatorname{Ind}_{Q_{P}}^{G} \sigma \otimes\left(\lambda_{0}+\mu\right) \otimes 1
\end{aligned}
$$

where we have identified, using $(2.27)$, $\mathfrak{a}_{P}^{\star}$ and $\mathfrak{a}_{P, 0}^{\star}$ with subspaces of $\mathfrak{a}^{\star}$.

- For the successive derivatives, we deduce that

$$
\pi_{P, \delta, \lambda_{0}}^{(\eta)} \simeq \text { subrepresentation of } \pi_{Q_{P}, \sigma, \lambda_{0}+\mu}^{(\eta)} .
$$

In other words, if we define $\xi^{\prime}$ to be the 4-tuple $\left(Q_{P}, \sigma, \lambda_{0}+\mu, \eta\right) \in \mathcal{D}$, we have

$$
\pi_{\xi} \simeq \text { subrepresentation of } \pi_{\xi^{\prime}} .
$$

- On the other hand, the parabolic subgroups $P$ and $Q_{P}$ of $G$ are conjugate under the Weyl group of $G$ with respect to $A$, i.e there exists $w \in N_{K}\left(\mathfrak{a}_{0}\right)$ such that $Q_{P}=$ $w^{-1} P_{0} w$. This induces an intertwining operator from $\pi_{Q_{P}, \sigma, \lambda_{0}+\mu}$ to $\pi_{P_{0}, w \cdot \sigma, w \cdot\left(\lambda_{0}+\mu\right)}$ and implies, for the successive derivatives, that

$$
\pi_{Q_{P}, \sigma, \lambda_{0}+\mu}^{(\eta)} \simeq \pi_{P_{0}, w \cdot \sigma, w \cdot\left(\lambda_{0}+\mu\right)}^{(w \cdot \eta)}
$$

where $w$ acts on $\eta$ componentwise. Defining the 4-tuple $\xi_{0}=\left(P_{0}, w \cdot \sigma, w \cdot\left(\lambda_{0}+\mu\right), w\right.$. $\eta) \in \mathcal{D}_{P_{0}}$ and using (2.28), we deduce that

$$
\pi_{\xi} \simeq \text { subrepresentation of } \pi_{\xi_{0}} .
$$

- By additivity, this extends to the case where $\xi$ is a finite sequence $\left(\xi_{1}, \xi_{2}, \cdots, \xi_{N}\right) \in \mathcal{D}^{N}$ which proves that $(b) \Rightarrow(a)$. 
2.9. On the bottom of the spectrum of $\Delta_{\ell}$. From Kuga's formula (see Proposition 2.5 of [10]), one has

$$
\pi_{P_{i}, \delta_{i}, \alpha_{i}}\left(\Delta_{\ell}\right)=-\pi_{P_{i}, \delta_{i}, \alpha_{i}}\left(\widetilde{\Omega}_{G}\right)
$$

independently of the degree $\ell$. Moreover it is known from [38] that

$$
\delta_{i} \in \widehat{M}_{i}\left(\sigma_{\ell}\right) \Longrightarrow\left\|\operatorname{char}\left(\delta_{i}\right)\right\| \leq\left\|\rho_{i}\right\|
$$

and the equality holds only if $\mathfrak{h}_{i}$ is maximally compact (i.e $\mathfrak{g}$ does not have real roots relative to $\left.\mathfrak{h}_{i}\right)$. Since there is a discrete number of irreducible unitary representations $\delta_{i}$ of $M$ occurring in $L^{2}\left(M_{i}\right)$ with Harish-Chandra parameter contained in the closed ball defined by $\left\|\operatorname{char}\left(\delta_{i}\right)\right\| \leq\left\|\rho_{i}\right\|$, the set $\widehat{M}_{i}\left(\sigma_{\ell}\right)$ is finite. We define the real number

$$
\lambda_{\ell}(G / K)=\inf \left\{-\omega_{P_{i}, \delta_{i}, \sqrt{-1} \nu_{i}} \mid \delta_{i} \in \widehat{M}_{i}\left(\sigma_{\ell}\right), \nu_{i} \in \mathfrak{a}_{i, 0}^{\star}, 1 \leq i \leq s\right\} .
$$

In particular we have

(i) $\lambda_{\ell}(G / K) \geq 0$ for all $\ell($ by $(2.30))$,

(ii) $\lambda_{\ell}(G / K)=\inf \left\{\left\|\rho_{i}\right\|^{2}-\left\|\operatorname{char}\left(\delta_{i}\right)\right\|^{2} \mid \delta_{i} \in \widehat{M}_{i}\left(\sigma_{\ell}\right), 1 \leq i \leq s\right\}$ (by ( $2.30)$ ), and

(iii) $\lambda_{\ell}(G / K)=0 \Leftrightarrow 2 \ell \in\left[\operatorname{dim}_{\mathbf{R}}(G / K)+\operatorname{rk}_{\mathbf{C}}(K)-\operatorname{rk}_{\mathbf{C}}(G), \operatorname{dim}_{\mathbf{R}}(G / K)+\right.$ $\left.\operatorname{rk}_{\mathbf{C}}(G)-\operatorname{rk}_{\mathbf{C}}(K)\right]$ (by $\left.(2.7)\right)$.

The link with the bottom of the spectrum of $\Delta_{\ell}$ is given by the following proposition.

Proposition 2.32. The number $\lambda_{\ell}(G / K)$ equals the bottom of the spectrum of $\Delta \ell$

Proof. Let $\mu_{\ell}$ be the bottom of the spectrum of $\Delta_{\ell}$. By the Plancherel theorem (2.11) and Kuga's formula (2.29), we know that

$$
\lambda_{\ell}(G / K) \leq \mu_{\ell}
$$

Assume that $\lambda_{\ell}(G / K)<\mu_{\ell}$ and let $\varphi$ be a smooth real function with compact support in the interval $\left[\lambda_{\ell}(G / K), \mu_{\ell}\right]$. Then we have

$$
\varphi\left(\Delta_{\ell}\right) \equiv 0
$$

where

$$
\varphi\left(\Delta_{\ell}\right)=\int_{-\infty}^{+\infty} \widehat{\varphi}(t) e^{\sqrt{-1} t \Delta_{\ell}} d t .
$$

Now we choose $\delta_{i} \in \widehat{M}_{i}\left(\sigma_{\ell}\right)$ and $\nu_{i} \in \mathfrak{a}_{i, 0}^{\star}$ such that

$$
\lambda_{\ell}(G / K)<\left\|\rho_{i}\right\|^{2}-\left\|\operatorname{char}\left(\delta_{i}\right)\right\|^{2}+\left\|\nu_{i}\right\|^{2}<\mu_{\ell}
$$

and we pick a non zero $\ell$-form $f$ in $L^{2}\left(G / K, \mathcal{V}_{\ell}\right)$ such that

$$
\widehat{f}\left(\delta_{i}, \sqrt{-1} \nu_{i}\right) \neq 0
$$


and $\widehat{f}\left(\delta_{i}, \cdot\right)$ is continuous on $\sqrt{-1} \mathfrak{a}_{i, 0}^{\star}$. Then we deduce that

$$
\begin{aligned}
\widehat{\varphi\left(\Delta_{\ell}\right)} f\left(\delta_{i}, \sqrt{-1} \nu_{i}\right) & =\varphi\left(\left\|\rho_{i}\right\|^{2}-\left\|\operatorname{char}\left(\delta_{i}\right)\right\|^{2}+\left\|\nu_{i}\right\|^{2}\right) \widehat{f}\left(\delta_{i}, \sqrt{-1} \nu_{i}\right) \\
& \neq 0
\end{aligned}
$$

which is absurd.

In the case of functions, i.e when $\ell=0$, it is not difficult to check, using the Plancherel formula for functions, that

$$
\lambda_{0}(G / K)=\left\|\rho_{\mathfrak{a}_{0}}\right\|^{2} .
$$

However the bottom of the spectrum of $\Delta_{\ell}$ is not known in general (see the appendix for computations of $\lambda_{\ell}(G / K)$ in some examples).

2.10. Heat kernel for differential forms. Recall that the Laplacian on $G$ is the negative elliptic differential operator $\Delta$ on $G$ defined by

$$
\Delta=\widetilde{\Omega}_{G}-2 \widetilde{\Omega}_{K} .
$$

We denote by $P_{t}=e^{t \Delta}$ the fundamental solution of the corresponding heat equation on $G$

$$
\Delta \phi_{t}=\frac{\partial}{\partial t} \phi_{t}
$$

It is well known that

$$
\left(P_{t} f\right)\left(g_{0}\right)=\int_{G} p_{t}\left(g_{0}^{-1} g\right) f(g) d g \quad \forall f \in L^{2}(G), g_{0} \in G
$$

where $p_{t} \in L^{2}(G) \cap C^{\infty}(G)$ is the heat kernel on $G$ [6]. Similarly we may consider the heat equation for differential forms on $G / K$

$$
\Delta_{\ell} \phi_{t}=-\frac{\partial}{\partial t} \phi_{t}
$$

and the corresponding fundamental solution

$$
P_{t}^{\ell}=e^{-t \Delta_{\ell}} .
$$

The operator

$$
P_{t}^{\ell}: L^{2}\left(G / K, \mathcal{V}_{\ell}\right) \rightarrow L^{2}\left(G / K, \mathcal{V}_{\ell}\right)
$$

is a smoothing pseudo-differential operator commuting with the representation $\pi_{\ell}$ of $G$. Actually we have

$$
\left(P_{t}^{\ell} \phi\right)\left(g_{0}\right)=\int_{G} p_{t}^{\ell}\left(g_{0}^{-1} g\right)(\phi(g)) d g \quad \forall \phi \in L^{2}\left(G / K, \mathcal{V}_{\ell}\right), g_{0} \in G
$$

where

$$
p_{t}^{\ell}: G \rightarrow \operatorname{End}\left(\Lambda^{\ell} \mathfrak{s}\right)
$$

is a smooth map satisfying the covariance property

$$
p_{t}^{\ell}\left(k g k^{\prime}\right)=\sigma_{\ell}(k)^{-1} \circ p_{t}^{\ell}(g) \circ \sigma_{\ell}\left(k^{\prime}\right)^{-1} \forall g \in G, k, k^{\prime} \in K .
$$


We shall refer to $p_{t}^{\ell}$ as the heat kernel of $\ell$-forms on $G / K$ (see Section 2 of [6]). It is easy to see from (2.5) that

$$
e^{-t \Delta_{\ell}} \circ Q_{\ell}=Q_{\ell} \circ\left(e^{t \Delta} \otimes e^{2 t \widetilde{\Omega}_{K}}\right),
$$

and

$$
p_{t}^{\ell}(g)=\int_{K \times K} p_{t}\left(k^{-1} g k^{\prime}\right) \sigma_{\ell}(k) e^{2 t \widetilde{\Omega}_{K}} \sigma_{\ell}\left(k^{\prime}\right)^{-1} d k d k^{\prime} .
$$

Similarly, for each irreducible component $\sigma_{\ell}^{j}$ of the isotropy representation, we define the heat kernel $p_{t}^{\ell, j}$

$$
p_{t}^{\ell, j}(g) \circ \operatorname{pr}_{j}=\operatorname{pr}_{j} \circ p_{t}^{\ell}(g), \quad \forall g \in G .
$$

Write $p_{t}^{\ell, \perp}$ for the heat kernel corresponding to the projection $\Delta_{\ell}^{\perp}$ of $\Delta_{\ell}$ on the orthogonal complement of $\operatorname{Ker}\left(\Delta_{\ell}\right)$ in $L^{2}\left(G / K, \mathcal{V}_{\ell}\right)$. The heat kernel $p_{t}^{\ell, j, \perp}$ is defined accordingly. From the Plancherel formula for differential form (2.11), one can deduce an explicit formula for $p_{t}^{\ell, j, \perp}$ using spherical Fourier transform (see (8.6) of [26])

$$
=\sum_{i, \operatorname{dim}\left(\mathfrak{a}_{i}\right)>0} \sum_{\delta_{i} \in \widehat{M}_{i}\left(\sigma_{\ell}^{j}\right)} \frac{1}{\left|W_{i}\right|} \frac{1}{\operatorname{dim}\left(V_{\ell}^{j}\right)} \int_{\mathfrak{a}_{i, 0}^{\star}} e^{t \omega_{P_{i}, \delta_{i}, \sqrt{-1} \nu_{i}}} \Psi_{\delta_{i}, \nu_{i}}^{\ell, j}(g) \mathbf{c}_{\delta_{i}}\left(\sqrt{-1} \nu_{i}\right) d \nu_{i} .
$$

Moreover one can check that

$$
\begin{aligned}
\Delta_{\ell} p_{t}^{\ell, j} & =-\frac{\partial}{\partial t} p_{t}^{\ell, j} \\
\widehat{p_{t}^{\ell, j}}\left(\delta_{i}, \sqrt{-1} \nu_{i}\right) & =e^{t \omega_{P_{i}, \delta_{i}, \sqrt{-1} \nu_{i}} \operatorname{Id}_{V_{\ell}^{j}}} \\
p_{t}^{\ell, j} \star p_{t^{\prime}}^{\ell, j} & =p_{t+t^{\prime}}^{\ell, j} \text { for all } t, t^{\prime}>0 .
\end{aligned}
$$

It should be noted that the continuous heat kernel $p_{t}^{\ell, \perp}$ coincides with the full heat kernel $p_{t}^{\ell}$ whenever $\operatorname{rk}_{\mathbf{C}}(G)>\operatorname{rk}_{\mathbf{C}}(K)$ or $\ell \neq \frac{1}{2} \operatorname{dim}(G / K)$.

In the sequel, we shall use the following basic estimates of the heat kernel $h_{t}$ for functions on $G / K$ (Chapter $\mathrm{V}$ of [14] for $0<t<1$ and Section 3 of [24] for $t \geq 1$ ). There exist positive constants $C_{1}$ and $C_{2}$ such that, for all $g \in G$, we have

$$
h_{t}(g K) \leq C_{1} t^{-\frac{1}{2} \operatorname{dim}_{\mathbf{R}}(G / K)} e^{-\frac{\|g\|^{2}}{4 t}} \text { for } 0<t<1,
$$

and

$$
h_{t}(g K) \leq C_{2} t^{-\frac{1}{2} \mathrm{rk}_{\mathbf{R}}(G)-\left|\Sigma^{++}\right|} e^{-\|\left.\rho_{\mathrm{a}_{0}}\right|^{2} t} \text { for } t \geq 1 .
$$

On the other hand, there is a well known relation between $h_{t}$ and the heat kernel $p_{t}^{\ell}$ on $\ell$-forms. Indeed, there exists two positive numbers $\alpha_{\ell}$ and $C_{3}$ such that, for all $t>0$ and $g \in G$ (Lemme 2.4 in [22]), one has

$$
\left\|p_{t}^{\ell}(g)\right\|_{\operatorname{End}\left(\Lambda^{\ell} \mathfrak{s}\right)} \leq C_{3} e^{t \alpha_{\ell}} h_{t}(g K) .
$$

The heat operator on functions on $G / K$ will be denoted by $H_{t}$. 
2.11. Laplacian on square integrable differential forms on locally symmetric spaces. If $\Gamma$ is a torsion free discrete subgroup of $G$, it acts on the left on $G / K$, so that the double coset space $\Gamma \backslash G / K$ is locally symmetric. Except otherwise stated, we do not assume that $\Gamma$ is of finite covolume in $G$. Since $G / K$ is simply connected, it is the universal cover of $\Gamma \backslash G / K$ and $\Gamma \simeq \Pi_{1}(\Gamma \backslash G / K)$. Moreover, given a Haar measure on $G$, there exists a unique measure $d \nu$ on $\Gamma \backslash G$ such that

$$
\int_{G} f(g) d g=\int_{\Gamma \backslash G}\left[\sum_{\gamma \in \Gamma} f(\gamma g)\right] d \nu(\Gamma g)
$$

for all compactly supported function $f$ on $G$. A smooth $\ell$-form on $\Gamma \backslash G / K$ may be viewed as a smooth $\Lambda^{\ell} \mathfrak{s - v a l u e d}$ function $\phi$ on $G$ satisfying the relation

$$
\phi(\gamma g k)=\sigma_{\ell}(k)^{-1} \phi(g), \quad \forall g \in g, k \in K, \gamma \in \Gamma .
$$

Write $C_{0}^{\infty}\left(\Gamma \backslash G / K, \mathcal{V}_{\ell}\right)$ for the complex vector space of compactly supported smooth $\ell$-forms on $\Gamma \backslash G / K$. Similarly, we define the vector space $L^{p}\left(\Gamma \backslash G / K, \Lambda^{\ell} \mathfrak{s}\right)$ of $L^{p}$ integrable $\ell$-forms on $\Gamma \backslash G / K$, equipped with the norm

$$
\|\phi\|_{L^{p}\left(\Gamma \backslash G / K, \mathcal{V}_{\ell}\right)}=\left(\int_{\Gamma \backslash G}\|\phi(\Gamma g)\|_{\Lambda^{\ell_{\mathfrak{S}}}}^{p} d \nu(\Gamma g)\right)^{\frac{1}{p}}
$$

When $\ell=0$, i.e in the case of functions, we shall simply write $C_{0}^{\infty}(\Gamma \backslash G / K)$ and $L^{2}(\Gamma \backslash G / K)$. The space $L^{p}\left(\Gamma \backslash G / K, \mathcal{E} n d\left(\Lambda^{\ell} \mathfrak{s}\right)\right)$ of $\operatorname{End}\left(\Lambda^{\ell} \mathfrak{s}\right)$-valued $L^{p}$ functions on $\Gamma \backslash G / K$ is defined accordingly, with the norm

$$
\|\phi\|_{L^{p}\left(\Gamma \backslash G / K, \mathcal{E} n d\left(\Lambda^{\left.\ell_{\mathfrak{s}}\right)}\right)\right.}=\left(\int_{\Gamma \backslash G}\|\phi(g)\|_{\operatorname{End}\left(\Lambda^{\ell} \mathfrak{s}\right)}^{p} d g\right)^{\frac{1}{p}} .
$$

Write

$$
d_{\ell}: C_{0}^{\infty}\left(\Gamma \backslash G / K, \mathcal{V}_{\ell}\right) \rightarrow C_{0}^{\infty}\left(\Gamma \backslash G / K, \mathcal{V}_{\ell+1}\right)
$$

for the exterior differential and

$$
d_{\ell}^{\star}: C_{0}^{\infty}\left(\Gamma \backslash G / K, \mathcal{V}_{\ell+1}\right) \rightarrow C_{0}^{\infty}\left(\Gamma \backslash G / K, \mathcal{V}_{\ell}\right)
$$

for its adjoint. Then, the locally invariant positive elliptic operator

$$
\widetilde{\Delta}_{\ell}=d_{\ell}^{\star} d_{\ell}+d_{\ell-1} d_{\ell-1}^{\star}
$$

is the Laplacian on compactly supported smooth $\ell$-forms on $\Gamma \backslash G / K$. This differential operator has a unique selfadjoint extension to $L^{2}\left(\Gamma \backslash G / K, \mathcal{V}_{\ell}\right)$ which will be also denoted by the same symbol $\widetilde{\Delta}_{\ell}$. In particular, we may also consider the heat equation on $\Gamma \backslash G / K$

$$
\widetilde{\Delta}_{\ell} \phi_{t}=-\frac{\partial}{\partial t} \phi_{t} .
$$

We shall write $\widetilde{P}_{t}^{\ell}$ for its fundamental solution and $\widetilde{p}_{t}^{\ell}$ for the corresponding heat kernel. The estimate $(2.38)$ is still true if we replace $p_{t}^{\ell}\left(\right.$ resp. $\left.h_{t}\right)$ by $\widetilde{p}_{t}^{\ell}\left(\right.$ resp. $\left.\widetilde{h}_{t}^{\ell}\right)$ where $\widetilde{h}_{t}^{\ell}$ (resp. $\widetilde{H}_{t}$ ) denotes the heat kernel (resp. heat operator) on functions on $\Gamma \backslash G / K$. Similarly we define $\widetilde{p}_{t}^{\ell, \perp}$ and $\widetilde{h}_{t}^{\ell, \perp}$. 
2.12. On the bottom of the spectrum of $\widetilde{\Delta}_{\ell}$. Let $\beta_{\ell}(\Gamma \backslash G / K)$ be the bottom of the $L^{2}$-spectrum of $\widetilde{\Delta}_{\ell}$ :

$$
\begin{array}{r}
\beta_{\ell}(\Gamma \backslash G / K)=\inf \left\{\left\langle\widetilde{\Delta}_{\ell} f, f\right\rangle_{L^{2}\left(\Gamma \backslash G / K, \Lambda^{\mathfrak{s}^{\star}}\right.} \mid f \in C_{0}^{\infty}\left(\Gamma \backslash G / K, \Lambda^{\ell} \mathfrak{s}^{\star}\right),\right. \\
\left.\|f\|_{L^{2}\left(\Gamma \backslash G / K, \Lambda^{\ell} \mathfrak{s}^{\star}\right)}=1\right\} .
\end{array}
$$

In the case of functions, i.e $\ell=0$, some estimates for $\beta_{0}(\Gamma \backslash G / K)$ have been proved by E. Leuzinger [21]. More precisely, let $D($,$) be the Riemannian distance on G / K$ induced by the Killing form. The Poincaré series associated with $\Gamma$ is given, for all $x, x^{\prime} \in G / K$ and $s \in \mathbf{R}$, by

$$
\mathcal{P}_{s}\left(x, x^{\prime}\right)=\sum_{\gamma \in \Gamma} e^{-s D\left(x, \gamma x^{\prime}\right)} .
$$

Then the critical exponent of $\Gamma$ is the real number $\delta(\Gamma)$ defined as follows. For all $x, x^{\prime} \in G, P_{s}\left(x, x^{\prime}\right)$ converges for $s>\delta(\Gamma)$ and diverges for $s<\delta(\Gamma)$, i.e

$$
\delta(\Gamma)=\inf \left\{s \mid \sum_{\gamma \in \Gamma} e^{-s D\left(x, \gamma x^{\prime}\right)}<+\infty\right\} .
$$

It is not difficult to check that (see Section 2.2 of [21])

$$
0 \leq \delta(\Gamma) \leq 2\left\|\rho_{\mathfrak{a}_{0}}\right\|
$$

When $\Gamma$ is a lattice in $G$, it is known that (see Theorem 7.4 of [2])

$$
\delta(\Gamma)=2\left\|\rho_{\mathfrak{a}_{0}}\right\| .
$$

In the general case, we have the following result.

Theorem (E. Leuzinger, Section 1 of [21]). Let $G$ be a semisimple Lie group without compact factors and with trivial center. Let $\rho_{\text {min }}$ be the positive real number defined by

$$
\rho_{\min }=\inf \left\{\rho_{\mathfrak{a}_{0}}(X) \mid X \in \overline{\mathfrak{a}_{0}^{+}},\|X\|=1\right\} .
$$

If $\Gamma$ is a torsion free discrete subgroup of $G$, the following estimates hold.

(i) If $\delta(\Gamma) \in\left[0, \rho_{\text {min }}\right]$ then $\beta_{0}(\Gamma \backslash G / K)=\left\|\rho_{\mathfrak{a}_{0}}\right\|^{2}$,

(ii) if $\delta(\Gamma) \in\left[\rho_{\min },\left\|\rho_{\mathfrak{a}_{0}}\right\|\right]$ then $\left\|\rho_{\mathfrak{a}_{0}}\right\|^{2}-\left(\delta(\Gamma)-\rho_{\min }\right)^{2} \leq \beta_{0}(\Gamma \backslash G / K) \leq \|$ $\rho_{\mathfrak{a}_{0}} \|^{2}$, and

(iii) if $\delta(\Gamma) \in\left[\left\|\rho_{\mathfrak{a}_{0}}\right\|, 2\left\|\rho_{\mathfrak{a}_{0}}\right\|\right]$ then

$$
\begin{aligned}
& \operatorname{Max}\left\{0 ;\left\|\rho_{\mathfrak{a}_{0}}\right\|^{2}-\left(\delta(\Gamma)-\rho_{\min }\right)^{2}\right\} \leq \beta_{0}(\Gamma \backslash G / K) \leq\left\|\rho_{\mathfrak{a}_{0}}\right\|^{2}-\left(\delta(\Gamma)-\left\|\rho_{\mathfrak{a}_{0}}\right\|\right. \\
& )^{2} \text {. }
\end{aligned}
$$

2.13. $L^{2}$-cohomology on $\Gamma \backslash G / K$. We do not assume that $\Gamma$ is of finite covolume in $G$. Let $W_{2, \ell}$ be the vector subspace of $L^{2}\left(\Gamma \backslash G / K, \mathcal{V}_{\ell}\right)$ defined, for $\ell \geq 0$, by

$$
W_{2, \ell}=\left\{\omega \in L^{2}\left(\Gamma \backslash G / K, \mathcal{V}_{\ell}\right) \mid\left\|d_{\ell} \omega\right\|_{L^{2}\left(\Gamma \backslash G / K, \mathcal{V}_{\ell}\right)}<+\infty\right\}
$$

It is easy to check that the kernel $\operatorname{Ker}\left(d_{\ell}\right)$ of $d_{\ell}$ is a subspace of $W_{2, \ell}$ which is closed in $L^{2}\left(\Gamma \backslash G / K, \mathcal{V}_{\ell}\right)$. However, the image $\operatorname{Im}\left(d_{\ell-1}\right)$ of $d_{\ell-1}$ need not be closed in 
$L^{2}\left(\Gamma \backslash G / K, \mathcal{V}_{\ell}\right)$. We are therefore led to define the unreduced $L^{2}$-cohomology group $H^{(\ell)}(\Gamma \backslash G / K)$ of degree $\ell$ of $\Gamma \backslash G / K$

$$
H^{(\ell)}(\Gamma \backslash G / K)=\operatorname{Ker}\left(d_{\ell}\right) / \operatorname{Im}\left(d_{\ell-1}\right)
$$

and the reduced $L^{2}$-cohomology group $\bar{H}^{(\ell)}(\Gamma \backslash G / K)$ of degree $\ell$ of $\Gamma \backslash G / K$

$$
\bar{H}^{(\ell)}(\Gamma \backslash G / K)=\operatorname{Ker}\left(d_{\ell}\right) / \overline{\overline{\operatorname{Im}\left(d_{\ell-1}\right)}},
$$

where $\overline{\operatorname{Im}\left(d_{\ell-1}\right)}$ denotes the closure of $\operatorname{Im}\left(d_{\ell-1}\right)$ in $L^{2}\left(\Gamma \backslash G / K, \mathcal{V}_{\ell}\right)$. Observe that there is a natural surjection

$$
H^{(\ell)}(\Gamma \backslash G / K) \longrightarrow \bar{H}^{(\ell)}(\Gamma \backslash G / K)
$$

and

$$
\bar{H}^{(\ell)}(\Gamma \backslash G / K) \simeq \operatorname{Ker}\left(d_{\ell}\right) \cap \overline{\operatorname{Im}\left(d_{\ell-1}\right)}{ }^{\perp}=\operatorname{Ker}\left(\widetilde{\Delta}_{\ell}\right)
$$

where $\overline{\operatorname{Im}\left(d_{\ell-1}\right)} \perp$ denotes the orthogonal of $\overline{\operatorname{Im}\left(d_{\ell-1}\right)}$ in $L^{2}\left(\Gamma \backslash G / K, \mathcal{V}_{\ell}\right)$. When $\Gamma$ is cocompact, unreduced and reduced $L^{2}$-cohomologies coincide.

\section{Estimates for the heat kernel.}

THEOREM 3.1. Let $G$ be a non compact connected semisimple real Lie group with finite center and $K$ a maximal compact subgroup of $G$. Let $\lambda_{\ell}(G / K)$ be the bottom of the spectrum of $\Delta_{\ell}$ and $\Phi_{0}$ the Harish-Chandra spherical function on G. Put $r=\inf _{i}\left\{\operatorname{dim}_{\mathbf{R}}\left(\mathfrak{a}_{i, 0}\right)>0\right\}$ and $z=\inf _{i}\left\{\right.$ order of zero of $\mathbf{c}_{\delta_{i}}$ at $\left.\nu_{i}=0, \delta_{i} \in \widehat{M}_{i}\left(\sigma_{\ell}\right)\right\}$. Then, for all $\epsilon \in] 0,1\left[\right.$, there exist two positive numbers $a_{\epsilon}$ and $A_{\epsilon}$ such that

$$
\left\|p_{t}^{\ell, \perp}(g)\right\|_{E n d\left(\Lambda^{\mathfrak{e}_{\mathfrak{s}}}\right)} \leq a_{\epsilon} e^{-t \lambda_{\ell}(G / K)} \Phi_{0}(g) e^{-\frac{1-\epsilon}{(1+2 \epsilon)^{2}} \frac{\|g\|^{2}}{4 t}} t^{-\epsilon \frac{r+z}{2}}
$$

for all $g \in G$ and $t \in \mathbf{R}$ satisfying $\|g\|>A_{\epsilon}$ and $t>1$.

Proof. Throughout the proof the symbols $B_{j}$ and $C_{j}$ will denote positive real numbers.

Step 1: we reduce the problem. Since the Casimir operator $\widetilde{\Omega}_{K}$ of $K$ acts a a scalar operator on each irreducible component $\sigma_{\ell}^{j}$ of $\sigma_{\ell}$ and, any two irreducible components are orthogonal with respect to the scalar product $\langle,\rangle_{\Lambda^{\ell}}$, we may assume that $\eta$ and $\beta$ belong to the same irreducible component $\sigma_{\ell}^{j}$ for some $j$. Then, using the Cartan decomposition (2.1), we have

$$
\begin{aligned}
\left\langle p_{t}^{\ell}(g) \eta, \omega\right\rangle_{\Lambda^{\ell_{\mathfrak{S}}}} & =\left\langle p_{t}^{\ell, j}(g) \eta, \beta\right\rangle_{\Lambda^{\ell_{\mathfrak{s}}}} \\
& =\left\langle p_{t}^{\ell, j}\left(k_{1}(g) e^{a^{+}(g)} k_{2}(g)\right) \eta, \beta\right\rangle_{\Lambda^{\ell_{\mathfrak{S}}}} \\
& =\left\langle p_{t}^{\ell, j}\left(e^{a^{+}(g)}\right) \sigma_{\ell}^{j}\left(k_{2}(g)\right) \eta, \sigma_{\ell}^{j}\left(k_{1}(g)\right)^{-1} \beta\right\rangle_{\Lambda^{\ell_{\mathfrak{s}}}} \text { by }(2.33) .
\end{aligned}
$$

Therefore it is enough to consider $\left\langle p_{t}^{\ell, j, \perp}(a) \eta, \beta\right\rangle_{\Lambda^{\ell} \mathfrak{s}}$ for $a \in \exp \left(\overline{\mathfrak{a}_{0}^{+}}\right)$and $\eta, \beta \in V_{\ell}^{j}$. In particular, from (2.35), we obtain

$$
\begin{aligned}
& \left\langle p_{t}^{\ell, j, \perp}(a) \eta, \beta\right\rangle_{\Lambda^{\ell} \mathfrak{s}} \\
& =\sum_{i, \operatorname{dim}\left(\mathfrak{a}_{i}\right)>0} \sum_{\delta_{i} \in \widehat{M}_{i}\left(\sigma_{\ell}^{j}\right)} \frac{1}{\left|W_{i}\right|} \frac{1}{\operatorname{dim}\left(V_{\ell}^{j}\right)} \int_{\mathfrak{a}_{i, 0}^{\star}} e^{t \omega_{P_{i}, \delta_{i}, \sqrt{-1} \nu_{i}}\left\langle\Psi_{\delta_{i}, \nu_{i}}^{\ell, j}(a) \eta, \beta\right\rangle_{\Lambda^{\ell} \mathfrak{s}} \mathbf{c}_{\delta_{i}}\left(\sqrt{-1} \nu_{i}\right) d \nu_{i} .} .
\end{aligned}
$$


Put $\eta_{i, j}^{\ell}=T_{\delta_{i}}^{\ell, j \star} \eta$ and $\beta_{i, j}^{\ell}=T_{\delta_{i}}^{\ell, j \star} \beta$ so that $\eta_{i, j}^{\ell}$ and $\beta_{i, j}^{\ell}$ are two $K$-finite vectors in $\mathcal{H}_{\delta_{i}}$ with

$$
\begin{aligned}
& \int_{\mathfrak{a}_{i, 0}^{\star}} e^{t \omega_{P_{i}, \delta_{i}, \sqrt{-1} \nu_{i}}}\left\langle\Psi_{\delta_{i}, \nu_{i}}^{\ell, j}(a) \eta, \beta\right\rangle_{\Lambda^{\ell_{\mathfrak{S}}}} \mathbf{c}_{\delta_{i}}\left(\sqrt{-1} \nu_{i}\right) d \nu_{i} \\
& =\int_{\mathfrak{a}_{i, 0}^{\star}} e^{t \omega_{P_{i}, \delta_{i}, \sqrt{-1} \nu_{i}}}\left\langle\pi_{P_{i}, \delta_{i}, \sqrt{-1} \nu_{i}}(a) \eta_{i, j}^{\ell}, \beta_{i, j}^{\ell}\right\rangle_{\Lambda^{\ell} \mathfrak{S}} \mathbf{c}_{\delta_{i}}\left(\sqrt{-1} \nu_{i}\right) d \nu_{i} .
\end{aligned}
$$

Define the operator

$$
\eta_{i, j}^{\ell} \otimes \beta_{i, j}^{\ell}: \mathcal{H}_{\delta_{i}} \rightarrow \mathcal{H}_{\delta_{i}}, f \mapsto\left\langle\eta_{i, j}^{\ell}, f\right\rangle_{\mathcal{H}_{\delta_{i}}} \beta_{i, j}^{\ell}
$$

and write $\xi^{t}$ and $\xi_{\delta_{i}}^{t}$ for the complex functions

$$
\begin{aligned}
& \xi^{t}: \widehat{M}_{i}\left(\sigma_{\ell}^{j}\right) \times \mathfrak{a}_{i}^{\star} \rightarrow \mathbf{C},\left(\delta_{i}, \alpha_{i}\right) \mapsto e^{t \omega_{P_{i}, \delta_{i}, \alpha_{i}}} \\
& \xi_{\delta_{i}}^{t}: \mathfrak{a}_{i}^{\star} \rightarrow \mathbf{C}, \alpha_{i} \mapsto e^{t \omega_{P_{i}, \delta_{i}, \alpha_{i}}} .
\end{aligned}
$$

Consider the $\operatorname{End}\left(\mathcal{H}_{\delta_{i}}\right)$-valued map

$$
\phi_{i}^{t}: \widehat{M}_{i}\left(\sigma_{\ell}^{j}\right) \times \mathfrak{a}_{i}^{\star} \rightarrow \operatorname{End}\left(\mathcal{H}_{\delta_{i}}\right),\left(\delta_{i}, \alpha_{i}\right) \mapsto \xi^{t}\left(\delta_{i}, \alpha_{i}\right) \eta_{i, j}^{\ell} \otimes \beta_{i, j}^{\ell} .
$$

Following an idea of Alexopoulos and Lohoué [2], we decompose this map in two pieces. For this we fix a smooth function $\zeta: \mathbf{R} \rightarrow \mathbf{R}$ such that $\zeta(\tau)=1$ if $|\tau|>1$, and $\zeta$ vanishes in an neighborhood of the origin. Then, for $\epsilon \in] 0,1[$, define the function

$$
\zeta_{a}: \mathfrak{a}_{i}^{\star} \rightarrow \mathbf{R}, y \mapsto \zeta\left((1+\epsilon) \frac{\|y\|}{\|a\|}\right)
$$

and write

$$
\phi_{i}^{t}=\phi_{i, a}^{t}+\widetilde{\phi}_{i, a}^{t}
$$

with

$$
\begin{aligned}
& \phi_{i, a}^{t}\left(\delta_{i}, \alpha_{i}\right)=\left(\xi_{\delta_{i}}^{t} \star \widehat{\zeta_{a}}\right)\left(\alpha_{i}\right) \eta_{i, j}^{\ell} \otimes \beta_{i, j}^{\ell} \\
& \widetilde{\phi}_{i, a}^{t}\left(\delta_{i}, \alpha_{i}\right)=\left(\xi_{\delta_{i}}^{t} \star \widehat{1-\zeta_{a}}\right)\left(\alpha_{i}\right) \eta_{i, j}^{\ell} \otimes \beta_{i, j}^{\ell} .
\end{aligned}
$$


Here it should be noted that the convolution and the Fourier transform are defined on $\mathfrak{a}_{i}^{\star}$, in particular only the parabolic subgroup $P_{i}=M_{i} A_{i} N_{i}$ is involved. We have

$$
\begin{aligned}
& \left\langle p_{t}^{\ell, j, \perp}(a) \eta, \beta\right\rangle_{\Lambda^{\ell} \mathfrak{s}} \\
= & \sum_{i, \operatorname{dim}\left(\mathfrak{a}_{i}\right)>0} \sum_{\delta_{i} \in \widehat{M}_{i}\left(\sigma_{\ell}^{j}\right)} \frac{1}{\left|W_{i}\right|} \frac{1}{\operatorname{dim}\left(V_{\ell}^{j}\right)} \\
& \times \int_{\mathfrak{a}_{i, 0}^{\star}} e^{t \omega_{P_{i}, \delta_{i}, \sqrt{-1} \nu_{i}}\left\langle\pi_{P_{i}, \delta_{i}, \sqrt{-1} \nu_{i}}(a) \eta_{i, j}^{\ell}, \beta_{i, j}^{\ell}\right\rangle_{\mathcal{H}_{\delta_{i}}} \mathbf{c}_{\delta_{i}}\left(\sqrt{-1} \nu_{i}\right) d \nu_{i}} \\
= & \sum_{i, \operatorname{dim}\left(\mathfrak{a}_{i}\right)>0} \sum_{\delta_{i} \in \widehat{M}_{i}\left(\sigma_{\ell}^{j}\right)} \frac{1}{\left|W_{i}\right|} \frac{1}{\operatorname{dim}\left(V_{\ell}^{j}\right)} \\
& \times \int_{\mathfrak{a}_{i, 0}^{\star}} e^{t \omega_{P_{i}, \delta_{i}, \sqrt{-1} \nu_{i}}} \operatorname{Tr}\left(\pi_{P_{i}, \delta_{i}, \sqrt{-1} \nu_{i}}(a) \circ\left(\eta_{i, j}^{\ell} \otimes \beta_{i, j}^{\ell}\right)\right) \mathbf{c}_{\delta_{i}}\left(\sqrt{-1} \nu_{i}\right) d \nu_{i} \\
= & \sum_{i, \operatorname{dim}\left(\mathfrak{a}_{i}\right)>0} \sum_{\delta_{i} \in \widehat{M}_{i}\left(\sigma_{\ell}^{j}\right)} \frac{1}{\left|W_{i}\right|} \frac{1}{\operatorname{dim}\left(V_{\ell}^{j}\right)} \int_{\mathfrak{a}_{i, 0}^{\star}} \operatorname{Tr}\left(\pi_{P_{i}, \delta_{i}, \sqrt{-1} \nu_{i}}(a) \circ \phi_{i}^{t}\left(\delta_{i}, \sqrt{-1} \nu_{i}\right)\right) \mathbf{c}_{\delta_{i}}\left(\sqrt{-1} \nu_{i}\right) d \nu_{i} \\
= & \sum_{i, \operatorname{dim}\left(\mathfrak{a}_{i}\right)>0} \sum_{\delta_{i} \in \widehat{M}_{i}\left(\sigma_{\ell}^{j}\right)} \frac{1}{\left|W_{i}\right|} \frac{1}{\operatorname{dim}\left(\mathfrak{a}_{i}\right)>0} \int_{\delta_{i} \in \widehat{M}_{i}\left(\sigma_{\ell}^{j}\right)} \operatorname{Tr}\left(\pi_{P_{i}, \delta_{i}, \sqrt{-1} \nu_{i}}(a) \circ \phi_{i, a}^{t}\left(\delta_{i}, \sqrt{-1} \nu_{i}\right)\right) \mathbf{c}_{\delta_{i}}\left(\sqrt{-1} \nu_{i}\right) d \nu_{i} \\
+\sum_{i, 0} & \frac{1}{\operatorname{dim}\left(V_{\ell}^{j}\right)} \int_{\mathfrak{a}_{i, 0}^{\star}} \operatorname{Tr}\left(\pi_{P_{i}, \delta_{i}, \sqrt{-1} \nu_{i}}(a) \circ \widetilde{\phi}_{i, a}^{t}\left(\delta_{i}, \sqrt{-1} \nu_{i}\right)\right) \mathbf{c}_{\delta_{i}}\left(\sqrt{-1} \nu_{i}\right) d \nu_{i} .
\end{aligned}
$$

Observe that

$$
\widetilde{\phi}_{i, a}^{t}\left(\delta_{i}, \alpha_{i}\right)=\left(\xi_{\delta_{i}}^{t} \star \widehat{1-\zeta_{a}}\right)\left(\alpha_{i}\right) \xi_{\delta_{i}}^{-t}\left(\alpha_{i}\right) \phi_{i}^{t}\left(\delta_{i}, \alpha_{i}\right) .
$$

Consider the global maps

$$
\Phi^{t}=\Phi_{a}^{t}+\widetilde{\Phi}_{a}^{t} \in \bigoplus_{P_{i} \in \mathcal{P}(A)} \bigoplus_{\delta_{I} \in\left(\widehat{M}_{i}\right)_{d}} \mathcal{C}\left(\mathfrak{a}_{i}^{\star}\right) \otimes \operatorname{End}\left(C^{\infty}\left(K, \delta_{i}\right)\right)
$$

where

$$
\begin{aligned}
& \Phi_{a}^{t}\left(P_{i}, \delta_{i}, \alpha_{i}\right)=\phi_{i, a}^{t}\left(\delta_{i}, \alpha_{i}\right) \\
& \widetilde{\Phi}_{a}^{t}\left(P_{i}, \delta_{i}, \alpha_{i}\right)=\widetilde{\phi}_{i, a}^{t}\left(\delta_{i}, \alpha_{i}\right)
\end{aligned}
$$

Step 2: we apply Delorme's Paley-Wiener theorem to $\widetilde{\Phi}_{a}^{t}$.

* We start with the pre-Paley-Wiener condition $(\text { iii })_{r}$ in Section 2.8 for some $r$ which will be specified below. For $u, v \in \mathcal{H}_{\delta_{i}}^{\infty}$, one has

$$
\left\langle\widetilde{\phi}_{i, a}^{t}\left(\delta_{i}, \sqrt{-1} \nu_{i}\right) u, v\right\rangle_{\mathcal{H}_{\delta_{i}}}=\widehat{\psi}_{i, a}^{t}\left(\sqrt{-1} \nu_{i}\right)
$$

where the function

$$
\psi_{i, a}^{t}: \sqrt{-1} \mathfrak{a}_{i, 0}^{\star} \rightarrow \mathbf{C}, \sqrt{-1} \nu_{i} \mapsto \xi^{t}\left(\delta_{i}, \sqrt{-1} \nu_{i}\right)\left(1-\zeta_{a}\right)\left(\sqrt{-1} \nu_{i}\right)\left\langle\eta_{i j}^{\ell}, u\right\rangle_{\mathcal{H}_{\delta_{i}}}\left\langle\beta_{i j}^{\ell}, v\right\rangle_{\mathcal{H}_{\delta_{i}}}
$$

is supported in the closed ball in $\mathfrak{a}_{i, 0}^{\star}$ of radius $R_{\epsilon}=\frac{1}{1+\epsilon}\|a\|$ and center the origin. Now the classical Paley-Wiener theorem implies that 
- $\widetilde{\phi}_{i, a}^{t}$ extends to an entire function on $\mathfrak{a}_{i}^{\star}$, in particular the map $\tilde{\Phi}_{a}^{t}$ is holomorphic in the variable $\alpha_{i}$, and

- for all integer $N \in \mathbf{N}$, we have

$$
\sup _{\alpha_{i} \in \mathfrak{a}_{i}^{\star}}\left(1+\left\|\alpha_{i}\right\|^{2}\right)^{N} e^{-R_{\epsilon}\left\|\operatorname{Re}\left(\alpha_{i}\right)\right\|}\left|\widehat{\psi}_{i, a}^{t}\left(\delta_{i}, \alpha_{i}\right)\right|<+\infty .
$$

Therefore, since $\widehat{M}_{i}\left(\sigma_{\ell}^{j}\right)$ is a finite set, one has for all $\theta_{1}, \theta_{2} \in \mathcal{U}(\mathfrak{k})$

$$
\begin{aligned}
& \sup _{\alpha_{i} \in \mathfrak{a}_{i}^{\star}, \delta_{i} \in \widehat{M}_{i}\left(\sigma_{\ell}^{j}\right)}\left(1+\left\|\alpha_{i}\right\|^{2}+\left\|\operatorname{char}\left(\delta_{i}\right)\right\|^{2}\right)^{N} e^{-R_{\epsilon}\left\|\operatorname{Re}\left(\alpha_{i}\right)\right\|} \\
& \times\left\|\pi_{\delta_{i}, \alpha_{i}}\left(\theta_{1}\right) \widetilde{\phi}_{i, a}^{t}\left(\delta_{i}, \alpha_{i}\right) \pi_{\delta_{i}, \alpha_{i}}\left(\theta_{2}\right)\right\|_{L_{c}\left(\mathcal{H}_{\delta_{i}, \alpha_{i}}\right)}<+\infty,
\end{aligned}
$$

where

$$
\begin{aligned}
& \left\|\pi_{\delta_{i}, \alpha_{i}}\left(\theta_{1}\right) \widetilde{\phi}_{i, a}^{t}\left(\delta_{i}, \alpha_{i}\right) \pi_{\delta_{i}, \alpha_{i}}\left(\theta_{2}\right)\right\|_{L_{c}\left(\mathcal{H}_{\delta_{i}, \alpha_{i}}\right)} \\
= & \sup _{\left\|\omega_{1}\right\|=\left\|\omega_{2}\right\|=1}\left|\left\langle\pi_{\delta_{i}, \alpha_{i}}\left(\theta_{1}\right) \widetilde{\phi}_{i, a}^{t}\left(\delta_{i}, \alpha_{i}\right) \pi_{\delta_{i}, \alpha_{i}}\left(\theta_{2}\right) \omega_{1}, \omega_{2}\right\rangle_{\mathcal{H}_{\delta_{i}}}\right| \\
= & \sup _{\left\|\omega_{1}\right\|=\left\|\omega_{2}\right\|=1}\left|\left\langle\widetilde{\phi}_{i, a}^{t}\left(\delta_{i}, \alpha_{i}\right) \pi_{\delta_{i}, \alpha_{i}}\left(\theta_{2}\right) \omega_{1}, \pi_{\delta_{i}, \alpha_{i}}\left(\theta_{1}\right) \omega_{2}\right\rangle_{\mathcal{H}_{\delta_{i}}}\right| .
\end{aligned}
$$

Then, by bi- $K$-equivariance (2.33) of the heat kernel, we deduce that

$$
\widetilde{\Phi}_{a}^{t} \in \bigoplus_{P_{i} \in \mathcal{P}(A)} \bigoplus_{\delta_{i} \in\left(\widehat{M}_{i}\right)_{d}} \mathcal{O}\left(\mathfrak{a}_{i}^{\star}\right) \otimes \operatorname{End}\left(\mathcal{S}\left(K, \delta_{i}\right)\right)
$$

and $\widetilde{\Phi}_{a}^{t}$ satisfies pre-Paley-Wiener condition (iii) $r$ for $r=R_{\epsilon}$.

$\star$ We turn now to the intertwining conditions (i) and (ii). By (3.3) we see that the map $\Phi^{t}$ is actually the Fourier transform $\Phi^{t}=\widehat{h}_{t}$ of the complex-valued function

$$
h_{t}: G \rightarrow \mathbf{C}, g \mapsto\left\langle p_{t}^{\ell, j, \perp}\left(g^{-1}\right) \eta, \beta\right\rangle_{\Lambda^{\ell} \mathfrak{s}} .
$$

Fix a basis $\left\{X_{j}\right\}$ of $\mathfrak{g}_{0}$ such that $\alpha_{i}=\sum_{j} \alpha_{i, j}\left\langle X_{j}, \cdot\right\rangle$ and choose a sequence $\left\{f_{n}\right\}$ of compactly supported smooth functions on $G$ with supports in some balls in $G$ such that (see [36])

$$
\begin{aligned}
& \lim _{n \rightarrow+\infty} f_{n}(g)=1, \forall g \in G, \text { and, } \exists C>0 \text { such that } \forall k_{j} \in \mathbf{N}, \forall g \in G, \\
& \mid\left(L\left(X_{j_{1}}\right)^{k_{1}} \cdots L\left(X_{j_{p}}\right)^{k_{p}} f_{n}\right)(g) \quad C \quad C \quad \text { and } \\
& \lim _{n \rightarrow+\infty}\left(L\left(X_{j_{1}}\right)^{k_{1}} \cdots L\left(X_{j_{p}}\right)^{k_{p}} f_{n}\right)(g)=0 .
\end{aligned}
$$

Then, we have for $u \in \mathcal{H}_{\delta_{i}}^{\infty}$

$$
\begin{aligned}
& \left\|{\widehat{f_{n} h_{t}}}_{(}\left(P_{i}, \delta_{i}, \alpha_{i}\right) u-\widehat{h}_{t}\left(P_{i}, \delta_{i}, \alpha_{i}\right) u\right\|_{\mathcal{H}_{\delta_{i}}} \\
= & \left\|\int_{G}\left(f_{n}(g)-1\right) h_{t}(g) \pi_{P_{i}, \delta_{i}, \alpha_{i}}(g) u d g\right\| \\
\leq & \int_{G}\left|f_{n}(g)-1\right|\left|\left\langle p_{t}^{\ell, j, \perp}\left(g^{-1}\right) \eta, \beta\right\rangle_{\Lambda^{\ell}}\right|\left\|\pi_{P_{i}, \delta_{i}, \alpha_{i}}(g) u\right\|_{\mathcal{H}_{P_{i}, \delta_{i}, \alpha_{i}}} d g .
\end{aligned}
$$

Now, since the Casimir operator $\widetilde{\Omega}_{K}$ acts on $V_{\ell}^{j}$ by a non-negative scalar $\sigma_{\ell}^{j}\left(\widetilde{\Omega}_{K}\right)$, we deduce from (2.34) that

$$
p_{t}^{\ell, j}\left(g^{-1}\right)=e^{2 t \sigma_{\ell}^{j}\left(\widetilde{\Omega}_{K}\right)} \int_{K \times K} p_{t}\left(k^{-1} g^{-1} k^{\prime}\right) \sigma_{\ell}(k) \sigma_{\ell}\left(k^{\prime}\right)^{-1} d k d k^{\prime} .
$$


But it is not difficult to check that (see Lemma 8 of [34])

$$
\left|\int_{K} p_{t}\left(k^{-1} g^{-1} k^{\prime}\right) d k d k^{\prime}\right| \leq C_{1}\left|p_{t+1}\left(g^{-1}\right)\right| \quad \forall g \in G, t>1
$$

and it is also well known that (see Theorem 1 of [32])

$$
\left|p_{t+1}\left(g^{-1}\right)\right| \leq C_{2} e^{-\frac{\|g\|^{2}}{4(t+1)}} \forall g \in G, t>1 .
$$

Therefore we have

$$
\left\|p_{t}^{\ell, j, \perp}\left(g^{-1}\right)\right\|_{\operatorname{End}\left(V_{\ell}^{j}\right)} \leq C_{3} e^{2 t \sigma_{\ell}^{j}\left(\widetilde{\Omega}_{K}\right)} e^{-\frac{\|g\|^{2}}{4(t+1)}} \forall g \in G, t>1 .
$$

On the other hand, one has (see Proposition 7.15 of [20])

$$
\left\|\pi_{P_{i}, \delta_{i}, \alpha_{i}}(g) u\right\|_{\mathcal{H}_{\delta_{i}, \alpha_{i}}} \leq C_{4} e^{\left\|\operatorname{Re}\left(\alpha_{i}\right)\right\|\|g\|} .
$$

Since the function $g \mapsto e^{-\frac{\|g\|^{2}}{4(t+1)}+\left\|\operatorname{Re}\left(\alpha_{i}\right)\right\|\|g\|}$ belongs to $L^{1}(G)$, we may apply the Lebesgue convergence theorem to see that

$$
\lim _{n \rightarrow+\infty}\left\|\widehat{f}_{n} \widehat{h}_{t}\left(P_{i}, \delta_{i}, \alpha_{i}\right) u-\widehat{h}_{t}\left(P_{i}, \delta_{i}, \alpha_{i}\right) u\right\|_{\mathcal{H}_{P_{i}, \delta_{i}, \alpha_{i}}}=0
$$

Similarly, we have

$$
\begin{aligned}
& \left\|\pi_{P_{i}, \delta_{i}, \alpha_{i}}\left(X_{j}\right) \widehat{f}_{n} h_{t}\left(P_{i}, \delta_{i}, \alpha_{i}\right) u-\pi_{P_{i}, \delta_{i}, \alpha_{i}}\left(X_{j}\right) \widehat{h}_{t}\left(P_{i}, \delta_{i}, \alpha_{i}\right) u\right\|_{\mathcal{H}_{P_{i}, \delta_{i}, \alpha_{i}}} \\
= & \left\|\int_{G} h_{t}(g)\left(f_{n}(g)-1\right) \pi_{P_{i}, \delta_{i}, \alpha_{i}}\left(X_{j}\right) \pi_{P_{i}, \delta_{i}, \alpha_{i}}(g) u d g\right\|_{\mathcal{H}_{P_{i}, \delta_{i}, \alpha_{i}}} \\
= & \left\|\left.\int_{G} h_{t}(g)\left(f_{n}(g)-1\right) \frac{d}{d s}\right|_{s=0} \pi_{P_{i}, \delta_{i}, \alpha_{i}}\left(\exp \left(s X_{j}\right) g\right) u d g\right\|_{\mathcal{H}_{P_{i}, \delta_{i}, \alpha_{i}}} \\
= & \left\|\left.\int_{G} \frac{d}{d s}\right|_{s=0} h_{t}\left(\exp \left(-s X_{j}\right) g\right)\left(f_{n}\left(\exp \left(-s X_{j}\right) g\right)-1\right) \pi_{P_{i}, \delta_{i}, \alpha_{i}}(g) u d g\right\|_{\mathcal{H}_{P_{i}, \delta_{i}, \alpha_{i}}} \\
\leq & \int_{G}\left|\left(L\left(X_{j}\right) h_{t}\right)(g)\left(f_{n}(g)-1\right)\right|\left\|\pi_{P_{i}, \delta_{i}, \alpha_{i}}(g) u\right\|_{\mathcal{H}_{P_{i}, \delta_{i}, \alpha_{i}}} d g \\
+ & \int_{G}\left|h_{t}(g)\right|\left|\left(L\left(X_{j}\right) f_{n}\right)(g)\right|\left\|\pi_{P_{i}, \delta_{i}, \alpha_{i}}(g) u\right\|_{\mathcal{H}_{P_{i}, \delta_{i}, \alpha_{i}}} d g,
\end{aligned}
$$

and, by iteration, the Lebesgue convergence theorem shows that

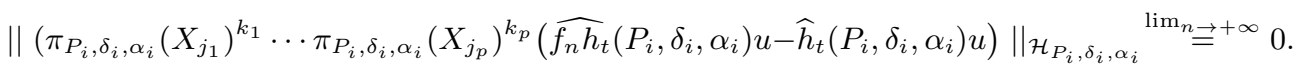

Therefore, since ${\widehat{f_{n} h_{t}}}_{\text {is }}$ compactly supported, it satisfies intertwining condition (i) so that if $V$ is a closed $G$-invariant subspace of $\mathcal{H}_{P_{i}, \delta_{i}, \alpha_{i}}$, then, we have that ${\widehat{f_{n} h_{t}}}_{(}\left(P_{i}, \delta_{i}, \alpha_{i}\right) V \subset V$. Taking the limit $n \rightarrow+\infty$ and using (3.4), we get that

$$
\widetilde{\Phi}_{a}^{t}\left(P_{i}, \delta_{i}, \alpha_{i}\right) V \subset V
$$

Next let $V_{1} \times V_{2}$ be a closed invariant subspace for $\frac{\partial}{\partial \lambda_{k}} \pi_{P_{i}, \delta_{i}, \alpha_{i}}$. Since $\widehat{f}_{n} \widehat{h}_{t}$ satisfies intertwining condition (i), the partial derivative $\frac{\partial}{\partial \lambda_{k}} \widehat{f_{n} h_{t}}$ leaves $V_{1} \times V_{2}$ invariant, 
using definition (2.20). Observing that

$$
\begin{aligned}
& {\left[\frac{\partial}{\partial \lambda_{k}} \widehat{f}_{n} h_{t}\left(P_{i}, \delta_{i}, \alpha_{i}\right)-\frac{\partial}{\partial \lambda_{k}} \widehat{h}_{t}\left(P_{i}, \delta_{i}, \alpha_{i}\right)\right]\left(v_{1}, v_{2}\right) } \\
= & \left(\widehat{f}_{n} h_{t}\left(P_{i}, \delta_{i}, \alpha_{i}\right) v_{1}+\alpha_{i, k} \widehat{f_{n} h_{t}}\left(P_{i}, \delta_{i}, \alpha_{i}\right) v_{2}, \widehat{f_{n} h_{t}}\left(P_{i}, \delta_{i}, \alpha_{i}\right) v_{2}\right) \\
- & \left(\widehat{h}_{t}\left(P_{i}, \delta_{i}, \alpha_{i}\right) v_{1}+\alpha_{i, k} \widehat{h}_{t}\left(P_{i}, \delta_{i}, \alpha_{i}\right) v_{2}, \widehat{h}_{t}\left(P_{i}, \delta_{i}, \alpha_{i}\right) v_{2}\right), \quad \forall\left(v_{1}, v_{2}\right) \in V_{1} \times V_{2}
\end{aligned}
$$

and using the same arguments as above, we deduce that $\frac{\partial}{\partial \lambda_{k}} \Phi^{t}$ leaves $V_{1} \times V_{2}$ invariant, i.e

$$
\begin{aligned}
& {\left[\frac{\partial}{\partial \lambda_{k}} \Phi^{t}\left(P_{i}, \delta_{i}, \alpha_{i}\right)\right]\left(v_{1}, v_{2}\right) } \\
= & \left(\left\langle\eta_{i, j}^{\ell}, v_{1}\right\rangle_{\mathcal{H}_{\delta_{i}}}+2 t \alpha_{i, k}\left\langle\eta_{i, j}^{\ell}, v_{2}\right\rangle_{\mathcal{H}_{\delta_{i}}}\right)\left(\beta_{i, j}^{\ell}, 0\right) \\
+ & \left\langle\eta_{i, j}^{\ell}, v_{2}\right\rangle_{\mathcal{H}_{\delta_{i}}}\left(0, \beta_{i, j}^{\ell}\right) \in V_{1} \times V_{2}, \quad \forall\left(v_{1}, v_{2}\right) \in V_{1} \times V_{2} .
\end{aligned}
$$

On the other hand, we have, for all $\left(v_{1}, v_{2}\right) \in V_{1} \times V_{2}$

$$
\begin{aligned}
& {\left[\frac{\partial}{\partial \lambda_{k}} \widetilde{\Phi}_{a}^{t}\left(P_{i}, \delta_{i}, \alpha_{i}\right)\right]\left(v_{1}, v_{2}\right) } \\
= & \left(\left(\xi_{\delta_{i}}^{t} \star\left(\widehat{1-\zeta_{a}}\right)\right)\left(\alpha_{i}\right)\left\langle\eta_{i, j}^{\ell}, v_{1}\right\rangle_{\mathcal{H}_{\delta_{i}}}+\frac{\partial}{\partial \lambda_{k}}\left(\xi_{\delta_{i}}^{t} \star\left(\widehat{1-\zeta_{a}}\right)\right)\left(\alpha_{i}\right)\left\langle\eta_{i, j}^{\ell}, v_{2}\right\rangle_{\mathcal{H}_{\delta_{i}}}\right)\left(\beta_{i, j}^{\ell}, 0\right) \\
+ & \left(\xi_{\delta_{i}}^{t} \star\left(\widehat{1-\zeta_{a}}\right)\right)\left(\alpha_{i}\right)\left\langle\eta_{i, j}^{\ell}, v_{2}\right\rangle_{\mathcal{H}_{\delta_{i}}}\left(0, \beta_{i, j}^{\ell}\right) .
\end{aligned}
$$

In particular, since both $\xi_{\delta_{i}}^{t}$ and $\xi_{\delta_{i}}^{t} \star\left(\widehat{1-\zeta_{a}}\right)$ are radial functions, $\frac{\partial}{\partial \lambda_{k}} \widetilde{\Phi}_{a}^{t}\left(P_{i}, \delta_{i}, 0\right)$ leaves $V_{1} \times V_{2}$ invariant. Indeed, when $\alpha_{i}=0$, one has

$$
\begin{aligned}
& {\left[\frac{\partial}{\partial \lambda_{k}} \widetilde{\Phi}_{a}^{t}\left(P_{i}, \delta_{i}, 0\right)\right]\left(v_{1}, v_{2}\right) } \\
= & \left(\xi_{\delta_{i}}^{t} \star\left(\widehat{1-\zeta_{a}}\right)\right)(0) \xi_{\delta_{i}}^{-t}(0)\left[\frac{\partial}{\partial \lambda_{k}} \Phi^{t}\left(P_{i}, \delta_{i}, 0\right)\right]\left(v_{1}, v_{2}\right) \in V_{1} \times V_{2} .
\end{aligned}
$$

Actually, if $\left\langle\eta_{i j}^{\ell}, v_{2}\right\rangle_{\mathcal{H}_{\delta_{i}}}=0$, and $\alpha_{i}$ need not be trivial, then

$$
\begin{aligned}
& {\left[\frac{\partial}{\partial \lambda_{k}} \widetilde{\Phi}_{a}^{t}\left(P_{i}, \delta_{i}, \alpha_{i}\right)\right]\left(v_{1}, v_{2}\right) } \\
= & \left(\xi_{\delta_{i}}^{t} \star\left(\widehat{1-\zeta_{a}}\right)\right)\left(\alpha_{i}\right) \xi_{\delta_{i}}^{-t}\left(\alpha_{i}\right)\left[\frac{\partial}{\partial \lambda_{k}} \Phi^{t}\left(P_{i}, \delta_{i}, \alpha_{i}\right)\right]\left(v_{1}, v_{2}\right) \in V_{1} \times V_{2} .
\end{aligned}
$$

If both $\alpha_{i} \neq 0$ and $\left\langle\eta_{i, j}^{\ell}, v_{2}\right\rangle_{\mathcal{H}_{\delta_{i}}} \neq 0$, then, applying (3.6) for two distinct $t_{1} \neq 0$ and $t_{2} \neq 0$, we see that both vectors $\left(\beta_{i, j}^{\ell}, 0\right)$ and $\left(0, \beta_{i, j}^{\ell}\right)$ belong to $V_{1} \times V_{2}$ which implies that $\left[\frac{\partial}{\partial \lambda_{k}} \widetilde{\Phi}_{a}^{t}\left(P_{i}, \delta_{i}, \alpha_{i}\right)\right]\left(v_{1}, v_{2}\right)$ belongs to $V_{1} \times V_{2}$. Therefore the first partial derivative of $\widetilde{\Phi}_{a}^{t}$ leaves invariant any closed invariant subspace $V_{1} \times V_{2}$ for $\frac{\partial}{\partial \lambda_{k}} \pi_{P_{i}, \delta_{i}, \alpha_{i}}$, i.e

$$
\left[\frac{\partial}{\partial \lambda_{k}} \widetilde{\Phi}_{a}^{t}\left(i, \delta_{i}, \alpha_{i}\right)\right] V_{1} \times V_{2} \subset V_{1} \times V_{2}
$$

Next let $\left(V_{1} \times V_{2}\right) \times\left(V_{3} \times V_{4}\right)$ be a closed invariant subspace of $\frac{\partial^{2}}{\partial \lambda_{k} \partial \lambda_{r}} \pi_{P_{i}, \delta_{i}, \alpha_{i}}$. Using the same argument as above we show that $\frac{\partial^{2}}{\partial \lambda_{k} \partial \lambda_{r}} \Phi^{t}$ leaves the subspace 
$\left(V_{1} \times V_{2}\right) \times\left(V_{3} \times V_{4}\right)$ invariant, i.e

$$
\begin{aligned}
& \left(\left\langle\eta_{i j}^{\ell}, v_{1}\right\rangle_{\mathcal{H}_{\delta_{i}}}+2 t \alpha_{i, r}\left\langle\eta_{i j}^{\ell}, v_{2}\right\rangle_{\mathcal{H}_{\delta_{i}}}+2 t \alpha_{i, k}\left\langle\eta_{i j}^{\ell}, v_{3}\right\rangle_{\mathcal{H}_{\delta_{i}}}\right. \\
& \left.+4 t^{2} \alpha_{i, k} \alpha_{i, r}\left\langle\eta_{i j}^{\ell}, v_{4}\right\rangle_{\mathcal{H}_{\delta_{i}}}+2 t \delta_{j k}\left\langle\eta_{i j}^{\ell}, v_{4}\right\rangle_{\mathcal{H}_{\delta_{i}}}\right)\left(\left(\beta_{i j}^{\ell}, 0\right),(0,0)\right) \\
+ & \left(\left\langle\eta_{i j}^{\ell}, v_{2}\right\rangle_{\mathcal{H}_{\delta_{i}}}+2 t \alpha_{i, k}\left\langle\eta_{i j}^{\ell}, v_{4}\right\rangle_{\mathcal{H}_{\delta_{i}}}\right)\left(\left(0, \beta_{i j}^{\ell}\right),(0,0)\right) \\
+ & \left(\left\langle\eta_{i j}^{\ell}, v_{3}\right\rangle_{\mathcal{H}_{\delta_{i}}}+2 t \alpha_{i, r}\left\langle\eta_{i j}^{\ell}, v_{4}\right\rangle_{\mathcal{H}_{\delta_{i}}}\right)\left((0,0),\left(\beta_{i j}^{\ell}, 0\right)\right) \\
+ & \left\langle\eta_{i j}^{\ell}, v_{4}\right\rangle_{\mathcal{H}_{\delta_{i}}}\left((0,0),\left(0, \beta_{i j}^{\ell}\right)\right) \\
\in & \left(V_{1} \times V_{2}\right) \times\left(V_{3} \times V_{4}\right)
\end{aligned}
$$

for all $\left(\left(v_{1}, v_{2}\right),\left(v_{3}, v_{4}\right)\right) \in\left(V_{1} \times V_{2}\right) \times\left(V_{3} \times V_{4}\right), \alpha \in \mathfrak{a}^{\star}$ and $t>0$. On the other hand, we have, by definition

$$
\begin{aligned}
& \frac{\partial^{2}}{\partial \lambda_{k} \partial \lambda_{r}} \widetilde{\Phi}_{a}^{t}\left(\left(v_{1}, v_{2}\right),\left(v_{3}, v_{4}\right)\right) \\
= & \left(\left(\xi_{\delta_{i}}^{t} \star\left(\widehat{1-\zeta_{a}}\right)\right)\left(\alpha_{i}\right)\left\langle\eta_{i j}^{\ell}, v_{1}\right\rangle_{\mathcal{H}_{\delta_{i}}}+\frac{\partial}{\partial \lambda_{r}}\left(\xi_{\delta_{i}}^{t} \star\left(\widehat{1-\zeta_{a}}\right)\right)\left(\alpha_{i}\right)\left\langle\eta_{i j}^{\ell}, v_{2}\right\rangle_{\mathcal{H}_{\delta_{i}}}\right. \\
+ & \frac{\partial}{\partial \lambda_{k}}\left(\xi_{\delta_{i}}^{t} \star\left(\widehat{1-\zeta_{a}}\right)\right)\left(\alpha_{i}\right)\left\langle\eta_{i j}^{\ell}, v_{3}\right\rangle_{\mathcal{H}_{\delta_{i}}} \\
+ & \left.\frac{\partial^{2}}{\partial \lambda_{k} \partial \lambda_{r}}\left(\xi_{\delta_{i}}^{t} \star\left(\widehat{1-\zeta_{a}}\right)\right)\left(\alpha_{i}\right)\left\langle\eta_{i j}^{\ell}, v_{4}\right\rangle_{\mathcal{H}_{\delta_{i}}}\right)\left(\left(\beta_{i j}^{\ell}, 0\right),(0,0)\right) \\
+ & \left(\left(\xi_{\delta_{i}}^{t} \star\left(\widehat{1-\zeta_{a}}\right)\right)\left(\alpha_{i}\right)\left\langle\eta_{i j}^{\ell}, v_{2}\right\rangle_{\mathcal{H}_{\delta_{i}}}+\frac{\partial}{\partial \lambda_{k}}\left(\xi_{\delta_{i}}^{t} \star\left(\widehat{1-\zeta_{a}}\right)\right)\left(\alpha_{i}\right)\left\langle\eta_{i j}^{\ell}, v_{4}\right\rangle_{\mathcal{H}_{\delta_{i}}}\right)\left(\left(0, \beta_{i j}^{\ell}\right),(0,0)\right) \\
+ & \left(\left(\xi_{\delta_{i}}^{t} \star\left(\widehat{1-\zeta_{a}}\right)\right)\left(\alpha_{i}\right)\left\langle\eta_{i j}^{\ell}, v_{3}\right\rangle_{\mathcal{H}_{\delta_{i}}}+\frac{\partial}{\partial \lambda_{r}}\left(\xi_{\delta_{i}}^{t} \star\left(\widehat{1-\zeta_{a}}\right)\right)\left(\alpha_{i}\right)\left\langle\eta_{i j}^{\ell}, v_{4}\right\rangle_{\mathcal{H}_{\delta_{i}}}\right)\left((0,0),\left(\beta_{i j}^{\ell}, 0\right)\right) \\
+ & \left(\left\langle\eta_{i j}^{\ell}, v_{3}\right\rangle_{\mathcal{H}_{\delta_{i}}}+2 t \alpha_{i, r}\left\langle\eta_{i j}^{\ell}, v_{4}\right\rangle_{\mathcal{H}_{\delta_{i}}}\right)\left((0,0),\left(\beta_{i j}^{\ell}, 0\right)\right) \\
+ & \left(\xi_{\delta_{i}}^{t} \star\left(\widehat{1-\zeta_{a}}\right)\right)\left(\alpha_{i}\right)\left\langle\eta_{i j}^{\ell}, v_{4}\right\rangle_{\mathcal{H}_{\delta_{i}}}\left((0,0),\left(0, \beta_{i j}^{\ell}\right)\right)
\end{aligned}
$$

Then, as in the case of the degree one above, by considering different values of $\alpha_{i}$ and $t$, we deduce that

$$
\begin{aligned}
& \frac{\partial^{2}}{\partial \lambda_{k} \partial \lambda_{r}} \Phi^{t}\left(P_{i}, \delta_{i}, \alpha_{i}\right)\left(\left(v_{1}, v_{2}\right),\left(v_{3}, v_{4}\right)\right) \in\left(V_{1} \times V_{2}\right) \times\left(V_{3} \times V_{4}\right) \\
& \Longrightarrow \frac{\partial^{2}}{\partial \lambda_{k} \partial \lambda_{r}} \widetilde{\Phi}_{a}^{t}\left(P_{i}, \delta_{i}, \alpha_{i}\right)\left(\left(v_{1}, v_{2}\right),\left(v_{3}, v_{4}\right)\right) \in\left(V_{1} \times V_{2}\right) \times\left(V_{3} \times V_{4}\right) .
\end{aligned}
$$

In a similar way, if $W$ is a closed invariant subspace for the successive partial derivative $\frac{\partial^{|q|}}{\partial \lambda_{j_{1}}^{q_{1}} \cdots \partial \lambda_{j_{p}}^{q_{p}}} \pi_{\delta, \lambda}$ of $\pi_{\delta, \lambda}$, we show that the successive partial derivative $\frac{\partial^{|q|}}{\partial \lambda_{j_{1}}^{q_{1}} \cdots \partial \lambda_{j_{p}}^{q_{p}}} \Phi^{t}$ leaves $W$ invariant. And so does the corresponding derivative of $\widetilde{\Phi}_{a}^{t}$

$$
\left[\frac{\partial^{|q|}}{\partial \lambda_{j_{1}}^{q_{1}} \cdots \partial \lambda_{j_{p}}^{q_{p}}} \widetilde{\Phi}_{a}^{t}\right] W \subset W
$$

In other words, the map $\widetilde{\Phi}_{a}^{t}$ satisfies intertwining condition (i), when $P_{i}$ is a minimal parabolic subgroup of $G$ in $\mathcal{P}(A)$. It should be noted that the fact both $\eta_{i, j}^{\ell}$ and $\beta_{i, j}^{\ell}$ do 
not depend on $\alpha_{i}$ and $t$ is essential in our arguments. Therefore, by Proposition 2.26, the map $\widetilde{\Phi}_{a}^{t}$ actually satisfies both Delorme's intertwining conditions (i) and (ii). In particular, the global map $\widetilde{\Phi}_{a}^{t}$ belongs to Delorme's Paley-Wiener space $\mathcal{P} \mathcal{W}_{R_{\epsilon}}(G, K)$ defined in (2.25). We apply Delorme's Paley-Wiener theorem to see that there exists a complex-valued function $\mu$ on $G$ which is supported in the closed ball in $\mathrm{G}$ of radius $R_{\epsilon}=\frac{1}{1+\epsilon}\|a\|$ and center the neutral element such that

$$
\begin{aligned}
& \sum_{i} \sum_{\delta_{i} \in \widehat{M}_{i}\left(\sigma_{\ell}^{j}\right)} \frac{1}{\left|W_{i}\right|} \frac{1}{\operatorname{dim}\left(V_{\ell}^{j}\right)} \int_{\mathfrak{a}_{i, 0}^{\star}} \operatorname{Tr}\left(\pi_{P_{i}, \delta_{i}, \sqrt{-1} \nu_{i}}(a) \circ \widetilde{\phi}_{i, a}^{t}\left(\delta_{i}, \sqrt{-1} \nu_{i}\right)\right) \mathbf{c}_{\delta_{i}}\left(\sqrt{-1} \nu_{i}\right) d \nu_{i} \\
& =\mu\left(a^{-1}\right) \\
& =0 .
\end{aligned}
$$

Step 3: we estimate the term in (3.3) involving $\Phi_{a}^{t}$. Recall that $\phi_{i, a}^{t}\left(\delta_{i}, \alpha_{i}\right)=$ $\left(\xi_{\delta_{i}}^{t} \star \widehat{\zeta}_{a}\right)\left(\alpha_{i}\right) \eta_{i, j}^{\ell} \otimes \beta_{i, j}^{\ell}$ so that, writing $\varphi_{t}\left(\nu_{i}\right)=e^{-t\left\|\nu_{i}\right\|^{2}}$, (3.3) implies that

$$
\begin{aligned}
& \left|\left\langle p_{t}^{\ell, j, \perp}(a) \eta, \beta\right\rangle_{\Lambda^{\ell} \mathfrak{s}}\right| \\
= & \mid \sum_{i} \sum_{\delta_{i} \in \widehat{M}_{i}\left(\sigma_{\ell}^{j}\right)} \frac{1}{\left|W_{i}\right|} \frac{1}{\operatorname{dim}\left(V_{\ell}^{j}\right)} \\
& \times \int_{\mathfrak{a}_{i, 0}^{\star}}\left\langle\pi_{P_{i}, \delta_{i}, \sqrt{-1} \nu_{i}}(a) \eta_{i, j}^{\ell}, \beta_{i, j}^{\ell}\right\rangle_{\mathcal{H}_{\delta_{i}}}\left(\xi^{\delta_{i}} \star \widehat{\zeta_{a}}\right)\left(\sqrt{-1} \nu_{i}\right) \mathbf{c}_{\delta_{i}}\left(\sqrt{-1} \nu_{i}\right) d \nu_{i} \mid \\
\leq & B_{1} e^{-t \lambda_{\ell}(G / K)} \Phi_{0}(a)\|\eta\|_{\Lambda^{\ell} \mathfrak{s}}\|\beta\|_{\Lambda^{\ell} \mathfrak{s}} \sup _{i} \int_{\mathfrak{a}_{i, 0}^{\star}}\left|\left(\varphi_{t} \star \widehat{\zeta_{a}}\right)\left(\nu_{i}\right)\right|^{2}\left(1+\left\|\nu_{i}\right\|^{2}\right)^{N_{i}} d \nu_{i}
\end{aligned}
$$

(by (2.8), (2.18) and the Cauchy-Schwartz inequality)

$$
\leq B_{2} e^{-t \lambda_{\ell}(G / K)} \Phi_{0}(a)\|\eta\|_{\Lambda^{\ell_{\mathfrak{s}}}}\|\beta\|_{\Lambda^{\ell_{\mathfrak{s}}}} \sup _{i}\left(\int_{\mathfrak{a}_{i, 0}^{\star}} \sum_{|q| \leq N_{i}}\left|\left(D^{q} \widehat{\varphi}_{t} \zeta_{a}\right)(z)\right|^{2} d z\right)^{\frac{1}{2}}
$$

(by equivalence with the Sobolev norm, where

$$
\begin{aligned}
& \left.D^{q}=\frac{\partial^{|q|}}{\partial x_{1}^{q_{1}} \cdots \partial x_{p}^{q_{p}}} \text { and }|q|=q_{1}+\cdots+q_{p}\right) \\
= & B_{2} e^{-t \lambda_{\ell}(G / K)} \Phi_{0}(a)\|\eta\|_{\Lambda^{\ell} \mathfrak{s}}\|\beta\|_{\Lambda^{\ell} \mathfrak{s}} \sup _{i}\left(\int_{\|z\| \geq \frac{1}{1+\epsilon}\|a\| \mid} \sum_{|q| \leq N_{i}}\left|\left(D^{q} \widehat{\varphi}_{t} \zeta_{a}\right)(z)\right|^{2} d z\right)^{\frac{1}{2}} \\
\leq & B_{3} e^{-t \lambda_{\ell}(G / K)} \Phi_{0}(a)\|\eta\|_{\Lambda^{\ell} \mathfrak{s}}\|\beta\|_{\Lambda^{\ell} \mathfrak{s}} e^{-\frac{\|a\| \|^{2}}{4(1+\epsilon)^{2} t}} \text { if } t>1 \\
& \left(\text { since } \widehat{\varphi}_{t}(z)=(2 t)^{-\frac{\operatorname{dim}\left(\mathfrak{a}_{i, 0}\right)}{2}} e^{-\frac{\|z\|^{2}}{4 t}}\right)
\end{aligned}
$$

and therefore

$$
\left\|p_{t}^{\ell, j, \perp}(a)\right\|_{\operatorname{End}\left(\Lambda^{\ell} \mathfrak{s}\right)} \leq b_{\epsilon, j} e^{-t \lambda_{\ell}(G / K)} \Phi_{0}(a) e^{-\frac{\|a\| \|^{2}}{4(1+\epsilon)^{2} t}}
$$

for some positive constant $b_{\epsilon, j}$ depending on both $\epsilon$ and $j$. Similarly we have

$$
\begin{aligned}
& \left|\left\langle p_{t}^{\ell, j, \perp}(a) \eta, \beta\right\rangle_{\Lambda^{\ell} \mathfrak{s}}\right| \\
\leq & B_{4} e^{-t \lambda_{\ell}(G / K)} \Phi_{0}(a)\|\eta\|_{\Lambda^{\ell} \mathfrak{s}}\|\beta\|_{\Lambda^{\ell_{\mathfrak{S}}}} \sup _{i} \int_{\mathfrak{a}_{i, 0}^{\star}} e^{-t\left\|\nu_{i}\right\|^{2}} \mathbf{c}_{\delta_{i}}\left(\sqrt{-1} \nu_{i}\right) d \nu_{i} .
\end{aligned}
$$


Now recall from (2.8) that there is a polynomial $P_{\delta_{i}}$ such that $\mathbf{c}_{\delta_{i}} \leq P_{\delta_{i}}$. Writing $P_{\delta_{i}}=\sum_{k \geq 0} a_{k} \nu_{i}^{k}$, we have

$$
\begin{aligned}
& \int_{\mathfrak{a}_{i, 0}^{\star}} e^{-t\left\|\nu_{i}\right\|^{2}} P_{\delta_{i}}\left(\nu_{i}\right) d \nu_{i} \\
= & \sum_{k \geq 0} a_{k} \int_{\mathfrak{a}_{i, 0}^{\star}} e^{-t|| \nu_{i} \|^{2}} \nu_{i}^{k} d \nu_{i} \\
= & \sum_{k \geq 0} a_{k} \int_{\left\|\nu_{i}\right\|=1} \nu_{i}^{k} d \nu_{i} \int_{0}^{+\infty} e^{-t r^{2}} r^{k+\operatorname{dim} \mathbf{R}_{\mathbf{R}}\left(\mathfrak{a}_{i, 0}\right)-1} d r \\
& \quad(\text { using spherical coordinates }) \\
= & t^{-\frac{\operatorname{dim}_{\mathbf{R}}\left(\mathfrak{a}_{i, 0}\right)}{2}} \sum_{k \geq 0} t^{-\frac{k}{2}} a_{k} \int_{\left\|\nu_{i}\right\|=1} \nu_{i}^{k} d \nu_{i} \int_{0}^{+\infty} e^{-r^{2}} r^{k+\operatorname{dim}_{\mathbf{R}}\left(\mathfrak{a}_{i, 0}\right)-1} d r .
\end{aligned}
$$

Then we deduce from (3.8) that

$$
\left\|p_{t}^{\ell, j, \perp}(a)\right\|_{\operatorname{End}\left(\Lambda_{\mathfrak{F}}\right)} \leq B_{5} e^{-t \lambda_{\ell}(G / K)} t^{-\frac{z+r}{2}} \Phi_{0}(a) \text { if } t>1
$$

where $z=\inf _{i}\left\{\right.$ order of zero of $\mathbf{c}_{\delta_{i}}$ at $\left.\nu_{i}=0, \quad \delta_{i} \in \widehat{M}_{i}\left(\sigma_{\ell}\right)\right\}$ and $r=$ $\inf _{i}\left\{\operatorname{dim}_{\mathbf{R}}\left(\mathfrak{a}_{i, 0}\right)>0\right\}$. Combining (3.7) and (3.9) we obtain

$$
\begin{aligned}
\left\|p_{t}^{\ell, j, \perp}(a)\right\|_{\operatorname{End}\left(\Lambda_{\mathfrak{F}}\right)} & =\left\|p_{t}^{\ell, j, \perp}(a)\right\|_{\operatorname{End}\left(\Lambda^{\ell_{\mathfrak{F}}}\right)}^{1-\frac{\epsilon}{\ell}}\left\|p_{t}^{\ell, j, \perp}(a)\right\|_{\operatorname{End}\left(\Lambda^{\ell_{\mathfrak{s}}}\right)}^{\frac{\epsilon}{2}} \\
& \leq c_{\epsilon, j} e^{-t \lambda_{\ell}(G / K)} \Phi_{0}(a) e^{-\frac{1-\frac{\epsilon}{2}}{(1+\epsilon)^{2}} \frac{\|a\|^{2}}{4 t}} t^{-\frac{\epsilon}{2} \frac{z+r}{2}} \text { if } t>1
\end{aligned}
$$

for some positive constant $c_{\epsilon, j}$ depending on both $\epsilon$ and $j$.

When $G$ has an empty discrete series, i.e $\operatorname{rk}_{\mathbf{C}}(G)>\operatorname{rk}_{\mathbf{C}}(K)$, the positive integer $r$ equals $\operatorname{rk}_{\mathbf{C}}(G)-\operatorname{rk}_{\mathbf{C}}(K)$ and the previous theorem may be restated as follows.

COROLlary 3.10. Under the assumptions of Theorem 3.1, if $G$ does not have discrete series representations then, for all $\epsilon \in] 0,1[$, there exist two positive numbers $A_{\epsilon}$ and $a_{\epsilon}$ such that

$$
\left\|p_{t}^{\ell, \perp}(g)\right\|_{E n d\left(\Lambda^{\ell} \mathfrak{s}\right)} \leq a_{\epsilon} e^{-t \lambda_{\ell}(G / K)} \Phi_{0}(g) e^{-\frac{1-\epsilon}{(1+2 \epsilon)^{2}} \frac{\|g\|^{2}}{4 t}} t^{-\epsilon \frac{z+r k_{\mathbf{C}}(G)-r k_{\mathbf{C}}(K)}{2}}
$$

for all $g \in G$ and $t \in \mathbf{R}$ satisfying $\|g\|>A_{\epsilon}$ and $t>1$.

If $G$ has an empty discrete series and is such that $z=0$ (as it is the case for the hyperbolic groups $G=S O_{e}(2 n+1,1)$, with $\left.n \geq 1\right)$, the exponent of $t$ in the estimate (3.11) has a nice geometric meaning. Indeed, in this case the (positive) integer $\operatorname{rk}_{\mathbf{C}}(G)-\operatorname{rk}_{\mathbf{C}}(K)$ is the $\ell$ th Novikov-Shubin invariant $a_{\ell}(\Gamma \backslash G / K)$ of the locally symmetric space $\Gamma \backslash G / K$, where $\Gamma$ is a torsion free discrete subgroup of $G$ of finite covolume (see (1.1)).

\section{Estimates for the resolvent.}

THEOREM 4.1. Let $G$ be a non compact connected semisimple real Lie group with finite center and $K$ a maximal compact subgroup of $G$. For a complex number 
$\mu$ with real part $\operatorname{Re}(\mu)$ and imaginary part $\operatorname{Im}(\mu)$ satisfying either $\operatorname{Im}(\mu) \neq 0$ or $\operatorname{Re}(\mu)<\lambda_{\ell}(G / K)$, define the positive number

$$
\tau_{\mu, \ell}(G / K)=\sqrt{\frac{\lambda_{\ell}(G / K)-\operatorname{Re}(\mu)+\sqrt{\left(\lambda_{\ell}(G / K)-\operatorname{Re}(\mu)\right)^{2}+\operatorname{Im}(\mu)^{2}}}{2}} .
$$

Then, for all $\epsilon \in] 0,1\left[\right.$, there exist two positive numbers $b_{\epsilon}$ and $B_{\epsilon}$ such that

$$
\left\|\left(\Delta_{\ell}-\mu\right)^{-1}(g)\right\| \leq b_{\epsilon} \Phi_{0}(g) e^{-(1-\epsilon) \tau_{\mu, \ell}(G / K)\|g\|}
$$

for all $g \in G$ satisfying $\|g\|>B_{\epsilon}$.

Proof. We follow the same strategy (and notation) as in the proof of the above theorem. Throughout the proof the symbols $B_{j}$ and $C_{j}$ will denote positive real numbers.

Step 1: Write $R_{\mu}^{\ell}$ for the resolvent operator $\left(\Delta_{\ell}-\mu\right)^{-1}$ of $\Delta_{\ell}$. For $\epsilon_{0}>0$ define $R_{\mu, \epsilon_{0}}^{\ell}=R_{\mu}^{\ell} \star P_{\epsilon_{0}}^{\ell}$, and the following functions

$$
\begin{aligned}
& \phi_{1, i}^{\epsilon_{0}}\left(\nu_{i}\right)=e^{-\epsilon_{0}\left\|\nu_{i}\right\|^{2}}, \quad \phi_{2, i}^{\epsilon_{0}}\left(\nu_{i}\right)=\frac{1}{-\omega_{P_{i}, \delta_{i}, \sqrt{-1} \nu_{i}}-\mu}, \\
& \text { and } \phi_{0, i}^{\epsilon_{0}}\left(\nu_{i}\right)=\phi_{2, i}^{\epsilon_{0}}\left(\nu_{i}\right) e^{\epsilon_{0} \omega_{P_{i}, \delta_{i}, \sqrt{ }-\nu_{i}}}
\end{aligned}
$$

Then we have, for all $a \in \exp \left(\overline{\mathfrak{a}^{+}}\right)$and $\eta, \beta \in V_{\ell}^{j}$

$$
\begin{aligned}
& \left\langle R_{\mu, \epsilon_{0}}^{\ell}(a) \eta, \beta\right\rangle_{\Lambda^{\ell} \mathfrak{s}} \\
= & \sum_{i} \sum_{\delta_{i} \in \widehat{M}_{i}\left(\sigma_{\ell}^{j}\right)} \frac{1}{\left|W_{i}\right|} \frac{1}{\operatorname{dim}\left(V_{\ell}^{j}\right)} \int_{\mathfrak{a}_{i, 0}^{\star}} \phi_{0, i}^{\epsilon_{0}}\left(\nu_{i}\right)\left\langle\Psi_{\delta_{i}, \nu_{i}}^{\ell, j}(a) \eta, \beta\right\rangle_{\Lambda^{\ell} \mathfrak{s}} \mathbf{c}_{\delta_{i}}\left(\sqrt{-1} \nu_{i}\right) d \nu_{i} \\
= & \sum_{i} \sum_{\delta_{i} \in \widehat{M}_{i}\left(\sigma_{\ell}^{j}\right)} \frac{1}{\left|W_{i}\right|} \frac{1}{\operatorname{dim}\left(V_{\ell}^{j}\right)} \int_{\mathfrak{a}_{i, 0}^{\star}} \operatorname{Tr}\left(\pi_{P_{i}, \delta_{i}, \sqrt{-1} \nu_{i}} \circ \phi_{i}^{\epsilon_{0}}\left(\delta_{i}, \sqrt{-1} \nu_{i}\right)\right) \mathbf{c}_{\delta_{i}}\left(\sqrt{-1} \nu_{i}\right) d \nu_{i} \\
& \text { where } \phi_{i}^{\epsilon_{0}}\left(\delta_{i}, \alpha_{i}\right)=\phi_{0, i}^{\epsilon_{0}}\left(\nu_{i}\right) \eta_{i, j}^{\ell} \otimes \beta_{i, j}^{\ell} \\
= & \sum_{i} \sum_{\delta_{i} \in \widehat{M}_{i}\left(\sigma_{\ell}^{j}\right)} \frac{1}{\left|W_{i}\right|} \frac{1}{\operatorname{dim}\left(V_{\ell}^{j}\right)} \int_{\mathfrak{a}_{i, 0}^{\star}} \operatorname{Tr}\left(\pi_{P_{i}, \delta_{i}, \sqrt{-1} \nu_{i}} \circ \phi_{i, a}^{\epsilon_{0}}\left(\delta_{i}, \sqrt{-1} \nu_{i}\right)\right) \mathbf{c}_{\delta_{i}}\left(\sqrt{-1} \nu_{i}\right) d \nu_{i} \\
& \text { using } \operatorname{Delorme} \operatorname{Theorem~with~} \phi_{i, a}^{\epsilon_{0}}\left(\delta_{i}, \alpha_{i}\right)=\left(\phi_{0, i}^{\epsilon_{0}} \star \zeta_{a}\right)\left(\nu_{i}\right) \eta_{i, j}^{\ell} \otimes \beta_{i, j}^{\ell} \\
& \text { and } \sum_{a} \text { is defined as in the proof of } \operatorname{Theorem~} 3.1 \text { above, } \\
& \sum_{i} \frac{1}{\mid W_{i} \in \widehat{M}_{i}\left(\sigma_{\ell}^{j}\right)} \frac{1}{\operatorname{dim}\left(V_{\ell}^{j}\right)} e^{\epsilon_{0}\left(\left\|\operatorname{char}\left(\delta_{i}\right)\right\|^{2}-\left\|\rho_{i}\right\|^{2}\right)} \\
& \times \int_{\mathfrak{a}_{i, 0}^{\star}}\left\langle\pi_{P_{i}, \delta_{i}, \sqrt{-1} \nu_{i}}(a) \eta_{i, j}^{\ell}, \beta_{i, j}^{\ell}\right\rangle_{\mathcal{H}_{\delta_{i}}}\left(\varphi_{\epsilon_{0}} \star \widehat{\zeta_{a}}\right)\left(\nu_{i}\right) \mathbf{c}_{\delta_{i}}\left(\sqrt{-1} \nu_{i}\right) d \nu_{i} \\
& \text { where } \varphi_{\epsilon_{0}}=\varphi_{1, i}^{\epsilon_{0}} \varphi_{2, i}
\end{aligned}
$$


so that

$$
\begin{aligned}
& \left|\left\langle R_{\mu, \epsilon_{0}}^{\ell}(a) \eta, \beta\right\rangle_{\Lambda^{\ell} \mathfrak{s}}\right| \\
\leq & B_{1} e^{-\epsilon_{0} \lambda_{\ell}(G / K)} \Phi_{0}(a)\|\eta\|_{\Lambda^{\ell_{\mathfrak{s}}}}\|\beta\|_{\Lambda^{\ell_{\mathfrak{s}}}} \sup _{i} \int_{\mathfrak{a}_{i, 0}^{\star}}\left|\left(\varphi_{\epsilon_{0}} \star \widehat{\zeta_{a}}\right)\left(\nu_{i}\right)\right|^{2}\left(1+\left\|\nu_{i}\right\|^{2}\right)^{N_{i}} d \nu_{i}
\end{aligned}
$$

(by (2.8), (2.18) and the Cauchy-Schwartz inequality)

$$
\leq B_{2} e^{-\epsilon_{0} \lambda_{\ell}(G / K)} \Phi_{0}(a) \sup _{i}\left(\int_{\mathfrak{a}_{i, 0}^{\star}} \sum_{\left|k_{1}\right|+\left|k_{2}\right| \leq N_{i}}\left|\left(D^{k_{1}}\left(\widehat{\varphi}_{1, i}^{\epsilon_{0}} \star \widehat{\varphi}_{2, i}\right) D^{k_{2}} \zeta_{a}\right)(z)\right|^{2} d z\right)^{\frac{1}{2}}
$$

(by equivalence with the Sobolev norm, where

$$
\left.D^{q}=\frac{\partial^{|q|}}{\partial x_{1}^{\mathfrak{q}_{1}} \cdots \partial x_{q_{p}}^{\mathfrak{q}_{p}}} \text { and }|q|=q_{1}+\cdots+q_{p}\right) .
$$

Step 2: Recall that $\widehat{\varphi_{1, i}^{\epsilon_{0}}}(z)=\left(2 \epsilon_{0}\right)^{-\frac{\operatorname{dim}\left(\mathfrak{a}_{i, 0}\right)}{2}} e^{-\frac{\|z \mid\|^{2}}{4 \epsilon_{0}}}$. Define the following family of complex numbers $\left\{\tau_{i}\right\}$ by

$$
\tau_{i}^{2}=\left\|\operatorname{char}\left(\delta_{i}\right)\right\|^{2}-\left\|\rho_{i}\right\|^{2}+\mu .
$$

Note that our assumptions on $\mu$ implies that the imaginary part of $\tau_{i}$ is positive. We deduce that

$$
\widehat{\varphi}_{2, i}(z)=\sqrt{-1} \frac{\pi}{4}\left(\|z\|^{-\operatorname{dim}\left(\mathfrak{a}_{i, 0}\right)+2}\left(\tau_{i}\|z\|\right)^{\frac{\operatorname{dim}\left(\mathfrak{a}_{i, 0}\right)}{2}-1} H_{\frac{\operatorname{dim}\left(\mathfrak{a}_{i, 0}\right)}{2}-1}^{(1)}\left(\tau_{i}\|z\|\right)\right.
$$

where $H_{\alpha}^{(1)}$ denotes the Bessel-Neumann function (see p. 65 of [29]). Indeed assume that $\tau_{i}=\sqrt{-1} r_{i}$ is imaginary with $r_{i}>0$, then

$$
\begin{aligned}
\widehat{\varphi}_{2, i}(z) & =\int_{\mathfrak{a}_{i, 0}^{\star}} \frac{e^{\sqrt{-1}\left\langle z, \nu_{i}\right\rangle}}{\left\|\nu_{i}\right\|^{2}+r_{i}^{2}} d \nu_{i} \\
& =\int_{\mathfrak{a}_{i, 0}^{\star}} e^{\sqrt{-1}\left\langle z, \nu_{i}\right\rangle} \int_{0}^{+\infty} e^{-t\left(\left\|\nu_{i}\right\|^{2}+r_{i}^{2}\right)} d t d \nu_{i} \\
& =\int_{0}^{+\infty} e^{-t r_{i}^{2}} \int_{\mathfrak{a}_{i, 0}^{\star}} e^{-t\left\|\nu_{i}\right\|^{2}} e^{\sqrt{-1}\left\langle z, \nu_{i}\right\rangle} d \nu_{i} d t \\
& =2^{-\frac{\operatorname{dim}\left(\mathfrak{a}_{i, 0}\right)}{2}} \int_{0}^{+\infty} e^{-t r_{i}^{2}} t^{-\frac{\operatorname{dim}\left(\mathfrak{a}_{i, 0}\right)}{2}} e^{-\frac{\|z\|^{2}}{4 t}} d t \\
& =\left(\frac{r_{i}}{\|z\|}\right)^{\frac{\operatorname{dim}\left(\mathfrak{a}_{i, 0}\right)}{2}-1} K_{\frac{\operatorname{dim}\left(\mathfrak{a}_{i, 0}\right)}{2}-1}\left(r_{i}\|z\|\right)
\end{aligned}
$$

(where $K_{\alpha}$ denotes the modified Bessel function, see p. 85 of [29]),

$$
\begin{aligned}
& =\sqrt{-1} \frac{\pi}{4}\left(\frac{\sqrt{-1} r_{i}}{\|z\|}\right)^{\frac{\operatorname{dim}\left(\mathfrak{a}_{i, 0}\right)}{2}-1} H_{\frac{\operatorname{dim}\left(\mathfrak{a}_{i, 0}\right)}{2}-1}^{(1)}\left(\sqrt{-1} r_{i}\|z\|\right) \\
& =\sqrt{-1} \frac{\pi}{4}\|z\|^{-\operatorname{dim}\left(\mathfrak{a}_{i, 0}\right)+2}\left(\sqrt{-1} r_{i}\|z\|\right)^{\frac{\operatorname{dim}\left(\mathfrak{a}_{i, 0}\right)}{2}-1} H_{\frac{\operatorname{dim}\left(\mathfrak{a}_{i, 0}\right)}{2}-1}^{(1)}\left(\sqrt{-1} r_{i}\|z\|\right),
\end{aligned}
$$

since (see p. 67 of [29])

$$
K_{\alpha}(z)=\frac{1}{2} \sqrt{-1} \pi e^{\sqrt{-1} \frac{\pi}{2} \alpha} H_{\alpha}^{(1)}\left(z e^{-\sqrt{-1} \frac{\pi}{2}}\right) .
$$


Now (4.4) follows by analytic continuation.

Step 3: We shall prove that there exists a positive constant $A_{i}$ that does not depend on $\epsilon_{0}$ so that

$$
\mid\left(D^{k_{1}}\left(\widehat{\varphi}_{1, i}^{\epsilon_{0}} \star \widehat{\varphi}_{2, i}\right)(z) \mid \leq A_{i} e^{-\operatorname{Im}\left(\tau_{i}\right)|| z||}\right.
$$

for $\|z\|$ sufficiently large. Indeed fix a smooth function $\zeta$ such that $\zeta(z)=1$ if $\|z\|>1$ and $\zeta(z)=0$ if $z$ belongs to some neighborhood of 0 . Write

$$
\widehat{\varphi}_{2, i}=\widehat{\varphi}_{2, i}^{1}+\widehat{\varphi}_{2, i}^{2}
$$

where $\widehat{\varphi}_{2, i}^{1}=(1-\zeta) \widehat{\varphi}_{2, i}$ and $\widehat{\varphi}_{2, i}^{2}=\zeta \widehat{\varphi}_{2, i}$. It turns out that

$$
D^{k_{1}} \widehat{\varphi}_{1, i}^{\epsilon_{0}}(z-y)=P_{k_{1}}(z-y) e^{-\frac{\|z-y\|^{2}}{4 \epsilon_{0}}}
$$

where $P_{k_{1}}(z-y)$ is a polynomial in $z-y$ with term of highest degree equal to $\left(2 \epsilon_{0}\right)^{-\left|k_{1}\right|-\frac{\operatorname{dim}\left(\mathfrak{a}_{i, 0}\right)}{2}}\left(z_{1}-y_{1}\right)^{k_{1}^{1}} \cdots\left(z_{\operatorname{dim}\left(\mathfrak{a}_{i, 0}\right)}-y_{\operatorname{dim}\left(\mathfrak{a}_{i, 0}\right)}\right)^{k_{1}^{\operatorname{dim}\left(\mathfrak{a}_{i, 0}\right)}}$ with $\left|k_{1}\right|=k_{1}^{1}+\cdots+$ $k_{1}^{\operatorname{dim}\left(\mathfrak{a}_{i, 0}\right)}$. We obtain successively

$$
\begin{aligned}
& \sup _{\|y\| \leq 1}\left|D^{k_{1}} \widehat{\varphi}_{1, i}^{\epsilon_{0}}(z-y)\right| \\
\leq & 2^{-\frac{\operatorname{dim}\left(\mathfrak{a}_{i, 0}\right)}{2}} \epsilon_{0}^{-\left|k_{1}\right|-\frac{\operatorname{dim}\left(\mathfrak{a}_{i, 0}\right)}{2}}\|z\|^{\left|k_{1}\right|} e^{-\frac{(\|z\|-1)^{2}}{4 \epsilon_{0}}}
\end{aligned}
$$

(since $2\|z\| \geq\|z-y\| \geq\|z\|-1$ for $\|z\|$ sufficiently large)$$
\leq 2^{-\frac{\operatorname{dim}\left(\mathfrak{a}_{i, 0}\right)}{2}}\|z\|^{-\left|k_{1}\right|} \epsilon_{0}^{-\left|k_{1}\right|-\frac{\operatorname{dim}\left(\mathfrak{a}_{i, 0}\right)}{2}}\|z\|^{2\left|k_{1}\right|} e^{-\frac{\|z\| \|^{2}}{16 \epsilon_{0}}}
$$$$
\text { (since }\|z\|-1 \geq \frac{\|z\|}{2} \text { for }\|z\| \geq 2 \text { ) }
$$$$
\leq 2^{-\left|k_{1}\right|-\frac{\operatorname{dim}\left(\mathfrak{a}_{i, 0}\right)}{2}} \epsilon_{0}^{-\left|k_{1}\right|-\frac{\operatorname{dim}\left(\mathfrak{a}_{i, 0}\right)}{2}}\|z\|^{2\left|k_{1}\right|} e^{-\frac{\|z \mid\|^{2}}{16 \epsilon_{0}}}
$$$$
\text { (for }\|z\| \geq 2 \text { ) }
$$$$
=2^{-\left|k_{1}\right|-\frac{\operatorname{dim}\left(\mathfrak{a}_{i, 0}\right)}{2}} w^{\left|k_{1}\right|} e^{-\frac{w}{32}} \epsilon_{0}^{-\frac{\operatorname{dim}\left(\mathfrak{a}_{i, 0}\right)}{2}} e^{-\frac{\| z||^{2}}{32 \epsilon_{0}}}
$$$$
\text { (writing } w=\frac{\|z\|^{2}}{\epsilon_{0}} \text { ) }
$$$$
\leq B_{1} 2^{-\left|k_{1}\right|-\frac{\operatorname{dim}\left(\mathfrak{a}_{i, 0}\right)}{2}} \epsilon_{0}^{-\frac{\operatorname{dim}\left(\mathfrak{a}_{i, 0}\right)}{2}} e^{-\frac{\|z \mid\|^{2}}{32 \epsilon_{0}}}
$$

(for some positive number $B_{1}$ that does not depend on $\epsilon_{0}$ )

$=B_{1} 2^{-\left|k_{1}\right|-\frac{\operatorname{dim}\left(\mathfrak{a}_{i, 0}\right)}{2}} \epsilon_{0}^{-\frac{\operatorname{dim}\left(\mathfrak{a}_{i, 0}\right)}{2}} e^{-\frac{\| z||^{2}}{64 \epsilon_{0}}} e^{-\frac{\|z\| \|^{2}}{64 \epsilon_{0}}}$

$\leq B_{2} 2^{-\left|k_{1}\right|-\frac{\operatorname{dim}\left(\mathfrak{a}_{i, 0}\right)}{2}} e^{-\frac{\|z\| \|^{2}}{64 \epsilon_{0}}}$

(for some positive number $B_{2}$ that does not depend on $\epsilon_{0}$ and for $\|z\| \geq 1$ )

$\leq B_{2} 2^{-\left|k_{1}\right|-\frac{\operatorname{dim}\left(\mathfrak{a}_{i, 0}\right)}{2}}(4 r)^{-\frac{\operatorname{dim}\left(\mathfrak{a}_{i, 0}\right)}{2}} e^{-\|z\|^{2}}$

(for $\epsilon_{0}$ sufficiently small). 
In particular we have, for $\|z\| \geq \operatorname{Im}\left(\tau_{i}\right)$

$$
\begin{aligned}
\mid\left(D^{k_{1}}\left(\widehat{\varphi}_{1, i}^{\epsilon_{0}} \star \widehat{\varphi}_{2, i}^{1}\right)(z) \mid\right. & =\left|\left(D^{k_{1}}\left(\widehat{\varphi}_{1, i}^{\epsilon_{0}}\right) \star \widehat{\varphi}_{2, i}^{1}\right)(z)\right| \\
& \left.\leq \sup _{\|y\| \leq 1}\left|D^{k_{1}} \widehat{\varphi}_{1, i}^{\epsilon_{0}}(z-y)\right| \int_{\|y\| \leq 1} \mid \widehat{\varphi}_{2, i}^{1}\right)(z) \mid d y \\
& \leq B_{3} e^{-\operatorname{Im}\left(\tau_{i}\right)\|z\|}
\end{aligned}
$$

(for some positive number $B_{3}$ that does not depend on $\epsilon_{0}$ ).

Step 4: We turn now to $D^{k_{1}}\left(\widehat{\varphi}_{1, i}^{\epsilon_{0}} \star \widehat{\varphi}_{2, i}^{2}\right)$. Combining the following relation satisfied by the modified Bessel functions (see p. 67 of [29])

$$
\left(\frac{d}{z d z}\right)^{m}\left[z^{-\gamma} K_{\gamma}(z)\right]=(-1)^{m} z^{-\gamma-m} K_{\gamma+m}(z), \quad m=1,2,3, \cdots
$$

along with (4.4), (4.5) and the asymptotics of $K_{\gamma}$ given on p. 139 of [29], we see that there exists a positive number $C_{1}$ such that

$$
\left|\left(D^{k_{1}} \widehat{\varphi}_{2, i}^{2}\right)(z)\right|=\left|\left(D^{k_{1}} \zeta \widehat{\varphi}_{2, i}\right)(z)\right| \leq C_{1} e^{-\operatorname{Im}\left(\tau_{i}\right)|| z||}
$$

for $\|z\|$ sufficiently large. Hence one has

$$
\begin{aligned}
\left|D^{k_{1}}\left(\widehat{\varphi}_{1, i}^{\epsilon_{0}} \star \widehat{\varphi}_{2, i}^{2}\right)(z)\right|= & \left|\widehat{\varphi}_{1, i}^{\epsilon_{0}} \star\left(D^{k_{1}} \widehat{\varphi}_{2, i}^{2}\right)(z)\right| \\
& \leq C_{1} \int_{\mathfrak{a}_{i, 0}^{\star}}\left(2 \epsilon_{0}\right)^{-\frac{\operatorname{dim}\left(a_{i, 0}\right)}{2}} e^{-\operatorname{Im}\left(\tau_{i}\right)\|z-y\|} e^{-\frac{\|y\|^{2}}{4 \epsilon_{0}}} d y \\
& =C_{1} 2^{\frac{\operatorname{dim}\left(a_{i, 0}\right)}{2}}\left(\int_{|| z\|\geq\| 2 \sqrt{\epsilon_{0}} w \|} e^{-\operatorname{Im}\left(\tau_{i}\right)\left\|z-2 \sqrt{\epsilon_{0}} w\right\|} e^{-\|w\|^{2}} d w\right. \\
& \left.+\int_{\|z\| \leq\left\|2 \sqrt{\epsilon_{0}} w\right\|} e^{-\operatorname{Im}\left(\tau_{i}\right)\left\|z-2 \sqrt{\epsilon_{0}} w\right\|} e^{-\|w\|^{2}} d w\right) \\
& \left(\text { writing } w=\frac{y}{2 \sqrt{\epsilon_{0}}}\right) .
\end{aligned}
$$

Now we have

$$
\begin{aligned}
& \int_{|| z|| \geq|| 2 \sqrt{\epsilon_{0}} w \|} e^{-\operatorname{Im}\left(\tau_{i}\right)\left\|z-2 \sqrt{\epsilon_{0}} w\right\|} e^{-\|w\|^{2}} d w \\
\leq & e^{-\operatorname{Im}\left(\tau_{i}\right)\|z\|} \int_{\|z\| \geq|| 2 \sqrt{\epsilon_{0}} w \|} e^{\operatorname{Im}\left(\tau_{i}\right)\left\|2 \sqrt{\epsilon_{0}} w\right\|} e^{-\|w\|^{2}} d w \\
\leq & e^{-\operatorname{Im}\left(\tau_{i}\right)\|z\|} \int_{\mathfrak{a}_{i, 0}^{\star}} e^{\operatorname{Im}\left(\tau_{i}\right)\|u\|} e^{-\frac{\|u\|^{2}}{4 \epsilon_{0}}} 2^{-\operatorname{dim}\left(\mathfrak{a}_{i, 0}\right)} \epsilon_{0}^{-\frac{\operatorname{dim}\left(\mathfrak{a}_{i, 0}\right)}{2}} d u \\
& \left(\operatorname{writing} u=2 \sqrt{\epsilon_{0}} w\right) \\
= & e^{-\operatorname{Im}\left(\tau_{i}\right)\|z\|} \int_{\|u\| \geq \operatorname{Im}\left(\tau_{i}\right)} e^{\operatorname{Im}\left(\tau_{i}\right)\|u\|} e^{-\frac{\|u\|^{2}}{4 \epsilon_{0}}} 2^{-\operatorname{dim}\left(\mathfrak{a}_{i, 0}\right)} \epsilon_{0}^{-\frac{\operatorname{dim}\left(\mathfrak{a}_{i, 0}\right)}{2}} d u \\
+ & e^{-\operatorname{Im}\left(\tau_{i}\right)\|z\|} \int_{\|u\| \leq \operatorname{Im}\left(\tau_{i}\right)} e^{\operatorname{Im}\left(\tau_{i}\right)\|u\|} e^{-\frac{\|u\|^{2}}{4 \epsilon_{0}}} 2^{-\operatorname{dim}\left(\mathfrak{a}_{i, 0}\right)} \epsilon_{0}^{-\frac{\operatorname{dim}\left(\mathfrak{a}_{i, 0}\right)}{2}} d u
\end{aligned}
$$


with

$$
\begin{aligned}
& \int_{\|u\| \geq \operatorname{Im}\left(\tau_{i}\right)} e^{\operatorname{Im}\left(\tau_{i}\right)\|u\| \mid} e^{-\frac{\|u\|^{2}}{4 \epsilon_{0}}} 2^{-\operatorname{dim}\left(\mathfrak{a}_{i, 0}\right)} \epsilon_{0}^{-\frac{\operatorname{dim}\left(\mathfrak{a}_{i, 0}\right)}{2}} d u \\
= & \int_{\|u\| \geq \operatorname{Im}\left(\tau_{i}\right)} e^{\operatorname{Im}\left(\tau_{i}\right)\|u\|} e^{-\frac{\|u\|^{2}}{8 \epsilon_{0}}} 2^{-\operatorname{dim}\left(\mathfrak{a}_{i, 0}\right)} \epsilon_{0}^{-\frac{\operatorname{dim}\left(a_{i, 0}\right)}{2}} e^{-\frac{\|u\|^{2}}{8 \epsilon_{0}}} d u \\
\leq & \sup _{\|u\| \geq \operatorname{Im}\left(\tau_{i}\right)} e^{\left(\operatorname{Im}\left(\tau_{i}\right)-\|u\|\right)\|u\|} \int_{\mathfrak{a}_{i, 0}^{\star}} 2^{-\operatorname{dim}\left(\mathfrak{a}_{i, 0}\right)} \epsilon_{0}^{-\frac{\operatorname{dim}\left(\mathfrak{a}_{i, 0}\right)}{2}} e^{-\frac{\|u\|^{2}}{8 \epsilon_{0}}} d u \\
= & \sup _{\|u\| \geq \operatorname{Im}\left(\tau_{i}\right)} e^{\left(\operatorname{Im}\left(\tau_{i}\right)-\|u\|\right)\|u\|} \int_{\mathfrak{a}_{i, 0}^{\star}} 2^{\frac{\operatorname{dim}\left(a_{i, 0}\right)}{2}} e^{-\|w\|^{2}} d w \\
& \left(\operatorname{writing} u=2^{\frac{3}{2}} \sqrt{\epsilon_{0}} w\right) \\
\leq & C_{2}
\end{aligned}
$$

and

$$
\begin{aligned}
& \int_{\|u\| \leq \operatorname{Im}\left(\tau_{i}\right)} e^{\operatorname{Im}\left(\tau_{i}\right)\|u\| \|} e^{-\frac{\|u\|^{2}}{4 \epsilon_{0}}} 2^{-\operatorname{dim}\left(\mathfrak{a}_{i, 0}\right)} \epsilon_{0}^{-\frac{\operatorname{dim}\left(\mathfrak{a}_{i, 0}\right)}{2}} d u \\
\leq & e^{\left(\operatorname{Im}\left(\tau_{i}\right)\right)^{2}} \int_{\mathfrak{a}_{i, 0}^{\star}} 2^{-\operatorname{dim}\left(\mathfrak{a}_{i, 0}\right)} \epsilon_{0}^{-\frac{\operatorname{dim}\left(\mathfrak{a}_{i, 0}\right)}{2}} e^{-\frac{\|u\|^{2}}{8 \epsilon_{0}}} d u \\
= & e^{\left(\operatorname{Im}\left(\tau_{i}\right)\right)^{2}} \int_{\mathfrak{a}_{i, 0}^{\star}} e^{-\|v\|^{2}} d v \\
& \left(\text { writing } u=2 \sqrt{\epsilon_{0}} v\right) \\
\leq & C_{3}
\end{aligned}
$$

so that

$$
\int_{\|z\| \geq|| 2 \sqrt{\epsilon_{0}} w \|} e^{-\operatorname{Im}\left(\tau_{i}\right)\left\|z-2 \sqrt{\epsilon_{0}} w\right\|} e^{-\|w\|^{2}} d w \leq C_{4} e^{-\operatorname{Im}\left(\tau_{i}\right)|| z \|}
$$

for $\|z\|$ sufficiently large. On the other hand, we have

$$
\begin{aligned}
& \int_{\|z\| \leq\left\|2 \sqrt{\epsilon_{0}} w\right\|} e^{-\operatorname{Im}\left(\tau_{i}\right)\left\|z-2 \sqrt{\epsilon_{0}} w\right\|} e^{-\|w\|^{2}} d w \\
\leq & e^{-\operatorname{Im}\left(\tau_{i}\right)\|z\|} \int_{\|z\| \leq\left\|2 \sqrt{\epsilon_{0}} w\right\|} e^{2 \operatorname{Im}\left(\tau_{i}\right)\left(\|z\|-\left\|\sqrt{\epsilon_{0}} w\right\|\right)} e^{-\|w\|^{2}} d w \\
\leq & e^{-\operatorname{Im}\left(\tau_{i}\right)\|z\|} e^{2 \operatorname{Im}\left(\tau_{i}\right)\|z\| \|} e^{-\frac{\|z\|^{2}}{4}} \\
\leq & C_{4} e^{-\operatorname{Im}\left(\tau_{i}\right)\|z\|}
\end{aligned}
$$

and thus

$$
\mid\left(D^{k_{1}}\left(\widehat{\varphi}_{1, i}^{\epsilon_{0}} \star \widehat{\varphi}_{2, i}^{2}\right)(z) \mid \leq C_{5} e^{-\operatorname{Im}\left(\tau_{i}\right)|| z \|}\right.
$$

for $\|z\|$ sufficiently large. Now (4.6) follows from (4.7), (4.8) and (4.9). 
Step 5: Finally we deduce that

$$
\begin{aligned}
& \left(\int_{\mathfrak{a}_{i, 0}^{\star}} \mid\left(\left.D^{k_{1}}\left(\widehat{\varphi}_{1, i}^{\epsilon_{0}} \star \widehat{\varphi}_{2, i}\right) D^{k_{2}} \zeta_{a}(z)\right|^{2} d z\right)^{\frac{1}{2}}\right. \\
\leq & \left(A_{i} \int_{\|z\| \geq \frac{\|a\|}{1+\epsilon}} e^{-2 \operatorname{Im}\left(\tau_{i}\right)\|z\|} d z\right)^{\frac{1}{2}} \\
= & A_{i}^{\frac{1}{2}}\left(\int_{\|z\| \geq \frac{\|a\| \mid}{1+\epsilon}} e^{-2(1-\epsilon) \operatorname{Im}\left(\tau_{i}\right)\|z\|} e^{-2 \epsilon \operatorname{Im}\left(\tau_{i}\right)\|z\|} d z\right)^{\frac{1}{2}} \\
\leq & A_{i}^{\frac{1}{2}} e^{-\frac{1-\epsilon}{1+\epsilon} \operatorname{Im}\left(\tau_{i}\right)\|a\|}\left(\int_{\|z\| \geq \frac{\|a\| \mid}{1+\epsilon}} e^{-2 \epsilon \operatorname{Im}\left(\tau_{i}\right)\|z\| d} d z\right)^{\frac{1}{2}} \\
= & D_{\epsilon, i} e^{-\frac{1-\epsilon}{1+\epsilon} \operatorname{Im}\left(\tau_{i}\right)\|a\|}
\end{aligned}
$$

(for some positive number $D_{\epsilon, i}$ depending on $\epsilon$ )

$$
\leq D_{\epsilon, i} e^{-(1-2 \epsilon) \operatorname{Im}\left(\tau_{i}\right)\|a\|} \text {. }
$$

In particular, for $\|a\|$ sufficiently large, (4.3) can be rewritten as follows

$$
\begin{aligned}
\left|\left\langle R_{\mu, \epsilon_{0}}^{\ell} \eta, \beta\right\rangle\right| & \leq B_{2} e^{-\epsilon_{0} \lambda_{\ell}(G / K)} \Phi_{0}(a) \sup _{i} D_{\epsilon, i} e^{-(1-2 \epsilon) \operatorname{Im}\left(\tau_{i}\right)\|a\|} \\
& \leq D_{\epsilon} e^{-\epsilon_{0} \lambda_{\ell}(G / K)} \Phi_{0}(a) e^{-(1-2 \epsilon)\|a\| \inf _{i}\left\{\operatorname{Im}\left(\tau_{i}\right)\right\}}
\end{aligned}
$$

(for some positive number $D_{\epsilon}$ depending on $\epsilon$ ).

The theorem follows by taking the limit $\epsilon_{0} \rightarrow 0$, since $D_{\epsilon}$ does not depend on $\epsilon_{0}$, and by observing that

$$
\operatorname{Im}\left(\tau_{i}\right)=\sqrt{\frac{\left\|\rho_{i}\right\|^{2}-\left\|\operatorname{char}\left(\delta_{i}\right)\right\|^{2}-\operatorname{Re}(\mu)+\sqrt{\left(\left\|\rho_{i}\right\|^{2}-\left\|\operatorname{char}\left(\delta_{i}\right)\right\|^{2}-\operatorname{Re}(\mu)\right)^{2}+\operatorname{Im}(\mu)^{2}}}{2}} .
$$

5. $L^{2+\epsilon}$-estimate for the resolvent of $\widetilde{\Delta}_{\ell}$. We start with some properties of the resolvent. Let $\Gamma$ be a torsion free discrete subgroup of $G$. The resolvent operator $R_{\mu}^{\ell}=\left(\Delta_{\ell}-\mu\right)^{-1}$ (resp. $\left.\widetilde{R}_{\nu}^{\ell}=\left(\widetilde{\Delta}_{\ell}-\nu\right)^{-1}\right)$, where $\mu$ (resp. $\nu$ ) is a complex number in the resolvent set of $\Delta_{\ell}$ (resp. $\left.\widetilde{\Delta}_{\ell}\right)$, is a kernel operator. Given a positive integer $k$, define (whenever the integrals converge) the maps

$$
\left(g_{1}, g_{2}\right) \in G \times G \mapsto \mathcal{R}_{\mu, k}^{\ell}\left(g_{1}, g_{2}\right)=\frac{1}{(k-1) !} \int_{0}^{+\infty} t^{k-1} e^{\mu t} p_{t}^{\ell}\left(g_{1}, g_{2}\right) d t
$$

and

$$
\left(\dot{g}_{1}, \dot{g}_{2}\right) \in \Gamma \backslash G \times \Gamma \backslash G \mapsto \widetilde{\mathcal{R}}_{\nu, k}^{\ell}\left(\dot{g}_{1}, \dot{g}_{2}\right)=\frac{1}{(k-1) !} \int_{0}^{+\infty} t^{k-1} e^{\nu t} \widetilde{p}_{t}^{\ell}\left(\dot{g}_{1}, \dot{g}_{2}\right) d t .
$$

When $k=1$, we shall simply write $\mathcal{R}_{\mu}^{\ell}$ and $\widetilde{\mathcal{R}}_{\nu}^{\ell}$ in stead of $\mathcal{R}_{\mu, 1}^{\ell}$ and $\widetilde{\mathcal{R}}_{\nu, 1}^{\ell}$ so that

$$
\widetilde{\mathcal{R}}_{\nu}^{\ell}\left(\dot{g}_{1}, \dot{g}_{2}\right)=\sum_{\gamma \in \Gamma} \mathcal{R}_{\nu}^{\ell}\left(g_{1}^{-1} \gamma g_{2}\right) .
$$

In the case of functions, i.e when $\ell=0$, write $S_{\mu}$ for the resolvent $\left(\Delta_{0}-\mu\right)^{-1}$ of the Laplacian $\Delta_{0}$ on $G / K$. The following estimate of $\mathcal{S}_{\mu}$, for $\|g\|>1$ and $\mu$ real such that $0 \leq \mu<\left\|\rho_{\mathfrak{a}_{0}}\right\|^{2}$ is due to Anker and Ji (Theorem 4.2.2 in [3]):

$$
\mathcal{S}_{\mu}(g) \asymp C \mu \Phi_{0}(g) e^{-\|g\| \sqrt{\left\|\rho_{\mathfrak{a}_{0}}\right\|^{2}-\mu}}
$$


for some positive constant $C_{\mu}$. Here $f_{1} \asymp f_{2}$ means that there exist two real numbers $C$ and $C^{\prime}$ such that $0<C \leq \frac{f_{1}(g)}{f_{2}(g)} \leq C^{\prime}$ for $\|g\|>1$. We will denote by $\widetilde{S}_{\nu}$ the resolvent $\left(\widetilde{\Delta}_{0}-\nu\right)^{-1}$ of the Laplacian $\widetilde{\Delta}_{0}$ on $\Gamma \backslash G / K$ so that

$$
S_{\nu}\left(\dot{g}_{1}, \dot{g}_{2}\right)=\sum_{\gamma \in \Gamma} S_{\nu}\left(g_{1}^{-1} \gamma g_{2}\right)
$$

The following two propositions are well known, however we were not able to find a precise reference for their proofs. Therefore, for the convenience of the reader, we shall provide a proof.

Proposition 5.2. (1) Assume $k$ is a positive integer and $\mu$ a negative real number. Let $1 \leq p \leq+\infty$ be an integer and write $p^{\prime}$ for the conjugate of $p$, i.e $\frac{1}{p}+\frac{1}{p^{\prime}}=1$. Then we have

(i) $\mathcal{R}_{\mu, k}^{\ell}(g, \cdot)$, for all $g \in G$, belongs to $L^{p}\left(G / K, \mathcal{E} n d\left(\Lambda^{\ell} \mathfrak{s}\right)\right)$ outside any ball centered at $g$ with finite radius, provided $\mu+\alpha_{\ell}-\frac{\left\|\rho_{\mathfrak{a}_{0}}\right\|^{2}}{p^{\prime}}<0$.

(ii) If $\operatorname{dim}_{\mathbf{R}}(G / K) \geq 2 k p^{\prime}$, then $\mathcal{R}_{\mu, k}^{\ell}(g, \cdot)$ belongs to $L^{p}\left(G / K, \mathcal{E} n d\left(\Lambda^{\ell} \mathfrak{s}\right)\right)$.

(2) Assume $\mu$ is a complex number such that: $\operatorname{Im}(\mu) \neq 0$ or $\operatorname{Re}(\mu)<\lambda_{\ell}(G / K)$. Then, outside any ball centered at the origin with finite radius, we have

(i) $\mathcal{R}_{\mu}^{\ell}(\cdot, e)$ belongs to $C^{\infty}\left(G / K, \mathcal{E} n d\left(\Lambda^{\ell} \mathfrak{s}\right)\right)$,

(ii) there exist a positive real number $C_{\mu}$ and a continuous function $\phi_{\mu}$, both depending on $\mu$, such that for all $g \in G$ satisfying $0<\|g\|<1$ :

$$
\begin{aligned}
& \left\|\mathcal{R}_{\mu}^{\ell}(g, e)\right\|_{E n d\left(\Lambda^{\ell} \mathfrak{s}\right)} \leq \frac{C_{\mu}}{\|g\|^{\operatorname{dim}_{\mathbf{R}}(G / K)-2}}+\phi_{\mu}(g), \quad \text { if } \operatorname{dim}_{\mathbf{R}}(G / K) \geq 3 \\
& \left\|\mathcal{R}_{\mu}^{\ell}(g, e)\right\|_{E n d\left(\Lambda^{\ell} \mathfrak{s}\right)} \leq C_{\mu} \log (\|g\|)+\phi_{\mu}(g), \text { if } \operatorname{dim}_{\mathbf{R}}(G / K)=2 .
\end{aligned}
$$

Proof. Throughout the proof $A_{j}$ will denote a positive real number. Let us start with the large time behavior. We have

$$
\begin{aligned}
\left\|\int_{1}^{+\infty} t^{k-1} e^{\mu t} p_{t}^{\ell}(g) d t\right\|_{\operatorname{End}\left(\Lambda^{\ell} \mathfrak{s}\right)} & \leq \int_{1}^{+\infty} t^{k-1} e^{\mu t}\left\|p_{t}^{\ell}(g)\right\|_{\operatorname{End}\left(\Lambda^{\ell} \mathfrak{s}\right)} d t \\
& \leq A_{1} \int_{1}^{+\infty} t^{k-1} e^{\left(\mu+\alpha_{\ell}\right) t} h_{t}(g) d t \text { by }(2.38) .
\end{aligned}
$$

Next, one has

$$
\begin{aligned}
\left\|h_{t}\right\|_{L^{1}(G / K)} & =1 \\
\left\|h_{t}\right\|_{L^{\infty}(G / K)} & \leq A_{2} t^{-\frac{1}{2} \mathrm{rk}_{\mathbf{R}}(G)-\left|\Sigma^{++}\right|} e^{-\left\|\rho_{\mathrm{a}_{0}}\right\|^{2} t} \text { by }(2.37) .
\end{aligned}
$$

Writing

$$
\frac{1}{p}=\frac{1-\theta}{1}+\frac{\theta}{\infty}
$$

for $\theta \in[0,1]$, we deduce, by interpolation, that

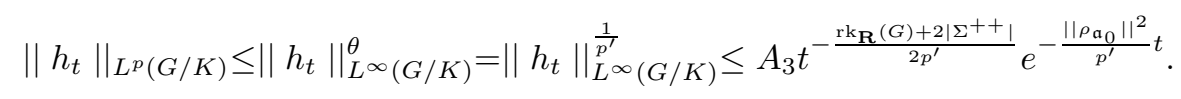


Therefore

$\left\|\int_{1}^{+\infty} t^{k-1} e^{\mu t} p_{t}^{\ell} d t\right\|_{L^{p}\left(G / K, \mathcal{E} n d\left(\Lambda^{\ell} \mathfrak{s}\right)\right)} \leq A_{3} \int_{1}^{+\infty} t^{k-1-\frac{\mathrm{rk}_{\mathbf{R}}(G)+2 \mid \Sigma^{++}}{2 p^{\prime}}} e^{\left(\mu+\alpha_{\ell}-\frac{\left\|\rho_{\boldsymbol{a}_{0}}\right\|^{2}}{p^{\prime}}\right) t} d t$ is finite if $\quad \mu+\alpha_{\ell}-\frac{\left\|\rho_{\mathfrak{a}_{0}}\right\|^{2}}{p^{\prime}}<0 \quad$ or $\quad$ if $\quad \mu+\alpha_{\ell}-\frac{\left\|\rho_{\mathfrak{a}_{0}}\right\|^{2}}{p^{\prime}}=0 \quad$ and $\operatorname{rk}_{\mathbf{R}}(G)+2\left|\Sigma^{++}\right|>2 k p^{\prime}$. Observe that the function

$$
g \mapsto \int_{1}^{+\infty} t^{k-1} e^{\left(\mu+\alpha_{\ell}\right) t} h_{t}(g) d t
$$

is bounded and continuous provided $\mu+\alpha_{\ell}-\left\|\rho_{\mathfrak{a}_{0}}\right\|^{2}<0$, which is the case if $\mu+\alpha_{\ell}-\frac{\left\|\rho_{\mathfrak{a}_{0}}\right\|^{2}}{p^{\prime}}<0$.

Similarly, we have

$$
\begin{aligned}
\left\|\int_{0}^{1} t^{k-1} e^{\mu t} p_{t}^{\ell}(g) d t\right\|_{\operatorname{End}\left(\Lambda^{\ell} \mathfrak{s}\right)} & \leq \int_{0}^{1} t^{k-1} e^{\mu t}\left\|p_{t}^{\ell}(g)\right\|_{\operatorname{End}\left(\Lambda^{\mathfrak{f}_{\mathfrak{S}}}\right)} d t \\
& \leq A_{4} \int_{0}^{1} t^{k-1} e^{\left(\mu+\alpha_{\ell}\right) t} h_{t}(g) d t \text { by }(2.38) \\
& \leq A_{5} \int_{0}^{1} t^{k-1-\frac{1}{2} \operatorname{dim}_{\mathbf{R}}(G / K)} e^{-\frac{\|g\|^{2}}{4 t}} d t \text { by }(2.36)
\end{aligned}
$$

and, by interpolation,

$$
\left\|\int_{0}^{1} t^{k-1} e^{\mu t} p_{t}^{\ell} d t\right\|_{L^{p}\left(G / K, \mathcal{E} n d\left(\Lambda^{\ell} \mathfrak{s}\right)\right)} \leq A_{6} \int_{0}^{1} t^{k-1-\frac{1}{2 p^{p}} \operatorname{dim}_{\mathbf{R}}(G / K)} d t
$$

which is finite if $\operatorname{dim}_{\mathbf{R}}(G / K)<2 k p^{\prime}$. This proves (1)(i) and (1)(ii).

We now turn to (2). We shall use the previous assertion. Fix a real number $\mu_{0}$ such that $\mu_{0}+\alpha_{\ell}<0$ and $\mathcal{R}_{\mu_{0}}^{\ell}(\cdot, e)$ is smooth outside any ball centered at the origin with finite radius. Iterating the basic relation

$$
R_{\mu}^{\ell}=R_{\mu_{0}}^{\ell}+\left(\mu-\mu_{0}\right) R_{\mu_{0}}^{\ell} \circ R_{\mu}^{\ell},
$$

we deduce that for all positive integer $N$

$$
R_{\mu}^{\ell}=\left(\mu-\mu_{0}\right)^{N} R_{\mu_{0}, N}^{\ell} \circ R_{\mu}^{\ell}+\sum_{j=1}^{N}\left(\mu-\mu_{0}\right)^{j} R_{\mu_{0}, j}^{\ell} .
$$

Let $\left\{X_{i}\right\}$ be a basis of $\mathfrak{g}_{0}$. Choose $N$ even and sufficiently large so that both $\mathcal{R}_{\mu_{0}, \frac{N}{2}}^{\ell}(\cdot, e)$ and $L\left(X_{j}\right)^{q} \mathcal{R}_{\mu_{0}, \frac{N}{2}}^{\ell}(\cdot, e)$ are $L^{2}$ on any ball centered at the origin with finite radius. Then, since $R_{\mu}^{\ell}$ is a bounded operator on $L^{2}\left(G / K, \Lambda^{\ell} \mathfrak{s}\right)$, we see that the convolution product $\mathcal{R}_{\mu_{0}, \frac{N}{2}}^{\ell}(\cdot, e) \star \mathcal{R}_{\mu}^{\ell}(\cdot, e)$ is square integrable on any ball centered at the origin with finite radius, so that

$$
L\left(X_{j}\right)^{q}\left(\mathcal{R}_{\mu_{0}, N}^{\ell}(\cdot, e) \star \mathcal{R}_{\mu}^{\ell}(\cdot, e)\right)=\left(L\left(X_{j}\right)^{q} \mathcal{R}_{\mu_{0}, \frac{N}{2}}^{\ell}(\cdot, e)\right) \star\left(\mathcal{R}_{\mu_{0}, \frac{N}{2}}^{\ell} \star \mathcal{R}_{\mu}^{\ell}(\cdot, e)\right)
$$


is continuous on such neighborhoods. The identity (5.4) implies that $L\left(X_{j}\right)^{q} \mathcal{R}_{\mu}^{\ell}(\cdot, e)$ is continuous which proves (2)(i). Finally, from (5.3), we see that

$$
\begin{aligned}
& \left\|\int_{0}^{1} t^{k-1} e^{\mu t} p_{t}^{\ell}(g) d t\right\|_{\operatorname{End}\left(\Lambda^{\ell} \mathfrak{s}\right)} \\
\leq & A_{6} \int_{0}^{1} t^{k-1-\frac{1}{2 p^{\prime}} \operatorname{dim}_{\mathbf{R}}(G / K)} e^{-\frac{\|g\|^{2}}{4 p^{\prime} t}} d t \\
\leq & A_{7}(p)\|g\|^{2 k-1-\operatorname{dim}_{\mathbf{R}}(G / K)} \quad \text { if } \operatorname{dim}_{\mathbf{R}}(G / K) \geq 2 k+1
\end{aligned}
$$

for some positive real number $A_{8}(p)$ depending on $p$ (after the change of variable $u=\frac{\|g\|^{2}}{4 p^{\prime} t}$ ). Now (2)(ii) follows by taking $k=1$, with the obvious modification if $\operatorname{dim}_{\mathbf{R}}(G / K)=2$.

Proposition 5.5. (1) Assume that $k$ and $p$ are positive integers with $k>\frac{1}{4} \operatorname{dim}_{\mathbf{R}}(G / K)$, and $\mu$ is a real number satisfying $\mu+\alpha_{\ell}<0$. Let $g$ be an element of $G$. Then, outside any ball centered at $\dot{g}$ with finite radius, $\widetilde{\mathcal{R}}_{\mu, k}^{\ell}(\dot{g}, \cdot)$ belongs to $L^{p}\left(\Gamma \backslash G / K, \mathcal{E} n d\left(\Lambda^{\ell} \mathfrak{s}\right)\right)$ and, there exists a positive real number $A(g)$ depending on $g$ such that

$$
\left\|\widetilde{\mathcal{R}}_{\mu, k}^{\ell}(\dot{g}, \cdot)\right\|_{L^{p}\left(\Gamma \backslash G / K, \mathcal{E} n d\left(\Lambda^{\ell} \mathfrak{s}\right)\right)} \leq A(g)\left(\inf \left\{1 ; \frac{\delta(\dot{g})}{2}\right\}\right)^{k-1-\left(\frac{p-1}{2 p}\right)\left(\operatorname{dim}_{\mathbf{R}}(G / K)-1\right)}
$$

where $\delta(\dot{g})$ denotes the injectivity radius of $\Gamma \backslash G / K$ at $\dot{g}$.

(2) Assume $q \geq 1$ and $\mu$ is a complex number satisfying $\operatorname{Im}(\mu) \neq 0$ or $\operatorname{Re}(\mu)<$ $\beta_{\ell}(\Gamma \backslash G / K)$. Then

(i) $\widetilde{\mathcal{R}}_{\mu, q}^{\ell}(\dot{g}, \cdot)$ is well defined, and

(ii) $\widetilde{\mathcal{R}}_{\mu, q}^{\ell}(\dot{g}, \cdot)$ belongs to $L^{2}\left(\Gamma \backslash G / K, \mathcal{E} n d\left(\Lambda^{\ell} \mathfrak{s}\right)\right)$. have:

Proof. Throughout the proof $A_{j}$ will denote a positive real number. First we

$$
\begin{aligned}
& \left\|\widetilde{\mathcal{R}}_{\mu, k}^{\ell}\left(\dot{g}_{1}, \cdot\right)\right\|_{L^{p}\left(\Gamma \backslash G / K, \mathcal{E} n d\left(\Lambda^{\ell} \mathfrak{s}\right)\right)} \\
\leq & \int_{0}^{+\infty} t^{k-1} e^{\mu t}\left\|\tilde{p}_{t}^{\ell}\left(\dot{g}_{1}, \cdot\right)\right\|_{L^{p}\left(\Gamma \backslash G / K, \mathcal{E} n d\left(\Lambda^{\ell} \mathfrak{s}\right)\right)} d t \\
\leq & A_{1} \int_{0}^{+\infty} t^{k-1} e^{\left(\mu+\alpha_{\ell}\right) t}\left\|\widetilde{h}_{t}\left(\dot{g}_{1}, \cdot\right)\right\|_{L^{p}(\Gamma \backslash G)} d t \text { by }(2.38) .
\end{aligned}
$$


Next, by the semigroup property of the heat kernel, we have

$$
\begin{aligned}
\left\|\widetilde{h}_{t}\left(\dot{g}_{1}, \cdot\right)\right\|_{L^{1}(\Gamma \backslash G / K)} & =\int_{\Gamma \backslash G} \widetilde{h}_{t}\left(\dot{g}_{1}, \dot{g}_{2}\right) d \nu\left(\dot{g}_{2}\right) \\
& =\int_{\Gamma \backslash G} \sum_{\gamma \in \Gamma} h_{t}\left(g_{1}^{-1} \gamma g_{2}\right) d \nu\left(\dot{g}_{2}\right) \\
& =\int_{G} h_{t}\left(g_{1}, g_{2}\right) d g_{2} \\
& =1 \\
\left\|\widetilde{h}_{t}\left(\dot{g}_{1}, \cdot\right)\right\|_{L^{\infty}(\Gamma \backslash G / K)} & =\sup _{\dot{g}_{2}} \widetilde{h}_{t}\left(\dot{g}_{1}, \dot{g}_{2}\right) \\
& =\widetilde{h}_{t}\left(\dot{g}_{1}, \dot{g}_{1}\right) \\
& =\left\|\widetilde{h}_{\frac{t}{2}}\left(\dot{g}_{1}, \cdot\right)\right\|_{L^{2}(\Gamma \backslash G / K)}^{2} \\
& \leq\left\|\widetilde{H}_{\frac{t}{2}-\frac{1}{2}}\right\|_{L^{2} \rightarrow L^{2}}^{2}\left\|\widetilde{h}_{\frac{1}{2}}\left(\dot{g}_{1}, \cdot\right)\right\|_{L^{2}(\Gamma \backslash G / K) \quad \text { for } t>1 .}^{2} .
\end{aligned}
$$

Writing $p \geq 1$ as

$$
\frac{1}{p}=\frac{1-\theta}{1}+\frac{\theta}{\infty} \text { for } \theta \in[0,1[
$$

we deduce that

$$
\begin{aligned}
\left\|\widetilde{h}_{t}\left(\dot{g}_{1}, \cdot\right)\right\|_{L^{p}(\Gamma \backslash G / K)} & \leq \widetilde{h}_{t}^{\theta}\left(\dot{g}_{1}, \dot{g}_{1}\right) \\
& \leq e^{-\theta \beta_{0}(\Gamma \backslash G / K) t} \widetilde{h}_{1}^{\theta}\left(\dot{g}_{1}, \dot{g}_{1}\right) \quad \text { if } t>1
\end{aligned}
$$

so that

$$
\left\|\widetilde{\mathcal{R}}_{\mu}^{k}\left(\dot{g}_{1}, \cdot\right)\right\|_{L^{p}\left(\Gamma \backslash G / K, \mathcal{E} n d\left(\Lambda^{\ell} \mathfrak{s}\right)\right)} \leq A_{1} \int_{0}^{+\infty} t^{k-1} e^{\left(\mu+\alpha_{\ell}\right) t} \widetilde{h}_{t}^{\theta}\left(\dot{g}_{1}, \dot{g}_{1}\right) d t .
$$

Hence we have

$$
\begin{aligned}
& \int_{0}^{+\infty} t^{k-1} e^{\left(\mu+\alpha_{\ell}-\theta \beta_{0}(\Gamma \backslash G / K)\right) t} \widetilde{h}_{1}^{\theta}\left(\dot{g}_{1}, \dot{g}_{1}\right) d t \\
\leq & A_{2} \int_{0}^{\inf \left\{1 ; \frac{\delta\left(\dot{g}_{1}\right)}{2}\right\}} t^{k-1-\frac{\theta}{2}\left(\operatorname{dim}_{\mathbf{R}}(G / K)+1\right)} d t \\
& (\text { by Theorem } 6 \text { of }[15]) \\
\leq & A_{3}\left(\inf \left\{1 ; \frac{\delta\left(\dot{g}_{1}\right)}{2}\right\}\right)^{k-1-\left(\frac{p-1}{2 p}\right)\left(\operatorname{dim}_{\mathbf{R}}(G / K)+1\right)} \\
& \left(\text { since } k \geq \frac{1}{4} \operatorname{dim}_{\mathbf{R}}(G / K)+1\right)
\end{aligned}
$$

and

$$
\begin{aligned}
& \int_{\inf \left\{1 ; \frac{\delta\left(\dot{g}_{1}\right)}{2}\right\}}^{+\infty} t^{k-1} e^{\left(\mu+\alpha_{\ell}\right) t} \widetilde{h}_{t}^{\theta}\left(\dot{g}_{1}, \dot{g}_{1}\right) d t \\
\leq & A_{4} \int_{\inf \left\{1 ; \frac{\delta\left(\dot{g}_{1}\right)}{2}\right\}}^{+\infty} t^{k-1} e^{\left(\mu+\alpha_{\ell}\right) t}\left\|\widetilde{H}_{\frac{t}{2}-\frac{1}{2}}\right\|_{L^{2} \rightarrow L^{2}}^{2 \theta}\left\|\widetilde{h}_{\frac{1}{2}}\left(\dot{g}_{1}, \cdot\right)\right\|_{L^{2}(\Gamma \backslash G / K)}^{2 \theta} d t \\
\leq & A_{5}\left(g_{1}\right) \text { a positive real number depending on } g_{1}
\end{aligned}
$$


which proves (1). For (2), we shall first consider the case where $q=1$. If $\mu_{0}$ is a real number satisfying $\mu_{0}+\alpha_{\ell}<0$, then

$$
\begin{aligned}
\left\|\int_{\Gamma \backslash G} \widetilde{\mathcal{R}}_{\mu_{0}}^{\ell}\left(\dot{g}_{1}, \dot{g}_{2}\right) f\left(\dot{g}_{2}\right) d \dot{g}_{2}\right\|_{\operatorname{End}\left(\Lambda^{\ell} \mathfrak{s}\right)} & =\left\|\int_{\Gamma \backslash G} \widetilde{\mathcal{R}}_{\mu_{0}, a}^{\ell}\left(\dot{g}_{1}, \dot{g}_{2}\right)\left(\widetilde{\Delta}_{\ell, \dot{g}_{2}}-\mu\right)^{a-1} f\left(\dot{g}_{2}\right) d \dot{g}_{2}\right\| \\
& \leq C(f)\left\|\widetilde{\mathcal{R}}_{\mu_{0}, a}^{\ell}\left(\dot{g}_{1}, \cdot\right)\right\|_{L^{2}\left(\Gamma \backslash G / K, \Lambda^{\ell} \mathfrak{s}\right)}
\end{aligned}
$$

for some positive constant depending on $f$. Choosing an integer $a \geq 1+\frac{1}{4} \operatorname{dim}_{\mathbf{R}}(G / K)$, we deduce from (1) that $\widetilde{\mathcal{R}}_{\mu_{0}}^{\ell}\left(\dot{g}_{1}, \cdot\right)$ is well defined outside any ball centered at $\dot{g}$ with finite radius. The same argument shows that $\widetilde{\mathcal{R}}_{\mu_{0}, k}^{\ell}$ is well defined on such neighborhoods for all $k \geq 1$. Next similarly to (5.4), we have

$$
\widetilde{R}_{\mu}^{\ell}=\left(\mu-\mu_{0}\right)^{N} \widetilde{R}_{\mu_{0}, N}^{\ell} \circ \widetilde{R}_{\mu}^{\ell}+\sum_{j=1}^{N}\left(\mu-\mu_{0}\right)^{j} \widetilde{R}_{\mu_{0}, j}^{\ell} .
$$

On the other hand, by the semigroup property of the heat kernel, one has

$$
\begin{aligned}
\widetilde{\mathcal{R}}_{\mu}^{\ell}\left(\dot{g}_{2}, \cdot\right) \widetilde{\mathcal{R}}_{\mu_{0}, N}^{\ell}\left(\dot{g}_{1}, \cdot\right) & =\int_{\Gamma \backslash G} \widetilde{\mathcal{R}}_{\mu_{0}, N}^{\ell}\left(\dot{g}_{1}, \dot{g}_{3}\right) \circ \widetilde{\mathcal{R}}_{\mu}^{\ell}\left(\dot{g}_{3}, \dot{g}_{2}\right) d \dot{g}_{3} \\
& =\int_{\Gamma \backslash G} \widetilde{\mathcal{R}}_{\mu_{0}, \frac{N}{2}}^{\ell}\left(\dot{g}_{1}, \dot{g}_{4}\right) \int_{\Gamma \backslash G} \widetilde{\mathcal{R}}_{\mu}^{\ell}\left(\dot{g}_{3}, \dot{g}_{2}\right) \circ \widetilde{\mathcal{R}}_{\mu_{0}, \frac{N}{2}}^{\ell}\left(\dot{g}_{4}, \dot{g}_{3}\right) d \dot{g}_{3} d \dot{g}_{4} .
\end{aligned}
$$

Fix $\dot{g}_{4}$ and choose an even integer $N$ sufficiently large such that $\dot{g}_{3} \mapsto \widetilde{\mathcal{R}}_{\mu_{0}, \frac{N}{2}}\left(\dot{g}_{4}, \dot{g}_{3}\right)$ belongs to $L^{2}\left(\Gamma \backslash G / K, \mathcal{E} n d\left(\Lambda^{\ell} \mathfrak{s}\right)\right)$ (by (1)). Then

$$
\int_{\Gamma \backslash G} \widetilde{\mathcal{R}}_{\mu}^{\ell}\left(\dot{g}_{3}, \dot{g}_{2}\right) \circ \widetilde{\mathcal{R}}_{\mu_{0}, \frac{N}{2}}^{\ell}\left(\dot{g}_{4}, \dot{g}_{3}\right) d \dot{g}_{3}=\widetilde{\mathcal{R}}_{\mu}^{\ell}\left(\dot{g}_{2}, \cdot\right) \widetilde{\mathcal{R}}_{\mu_{0}, \frac{N}{2}}^{\ell}\left(\dot{g}_{4}, \cdot\right) \in L^{2}\left(\Gamma \backslash G / K, \mathcal{E} n d\left(\Lambda^{\ell} \mathfrak{s}\right)\right),
$$

since $\widetilde{R}_{\mu}^{\ell}$ is a bounded operator on $L^{2}\left(\Gamma \backslash G / K, \Lambda^{\ell} \mathfrak{s}\right)$, and (5.6) implies that $\widetilde{\mathcal{R}}_{\mu}^{\ell}\left(\dot{g}_{1}, \cdot\right)$ is square integrable outside any ball centered at $\dot{g}$ with finite radius. Actually the same argument shows that $\dot{g}_{2} \mapsto \widetilde{\Delta}_{\ell, \dot{g}_{2}}^{a}\left(\widetilde{\mathcal{R}}_{\mu}^{1} \circ \widetilde{\mathcal{R}}_{\mu_{0}}^{N}\right)\left(\dot{g}_{1}, \dot{g}_{2}\right)$ belongs to $L^{2}\left(\Gamma \backslash G / K, \mathcal{E} n d\left(\Lambda^{\ell} \mathfrak{s}\right)\right)$ for all integer $a \geq 1$, so that $\widetilde{\mathcal{R}}_{\mu}^{\ell} \circ \widetilde{\mathcal{R}}_{\mu_{0}, N}^{\ell}$ is $C^{\infty}$ outside any ball centered at $\dot{g}$ with finite radius. Applying (5.6) we deduce that $\widetilde{\mathcal{R}}_{\mu}^{\ell}$ is $C^{\infty}$ on such neighborhoods. This proves (2)(i)(ii) for $q=1$. The case where $q \geq 2$ follows by induction from the following formula

$$
\widetilde{R}_{\mu, q}^{\ell}=\sum_{j=0}^{q} C_{q}^{j}\left(\mu-\mu_{0}\right)^{N j} \widetilde{R}_{\mu_{0}, N j} \circ \widetilde{R}_{\mu, j}^{\ell} \circ\left[\sum_{r=1}^{N}\left(\mu-\mu_{0}\right)^{r} \widetilde{R}_{\mu_{0}, r}^{\ell}\right]^{q-j}
$$

REMARK 5.8. If $\Gamma$ is of finite covolume in $G$, we may assume that the injectivity radius is small enough so that $\operatorname{Min}\left\{1 ; \frac{\delta\left(\dot{g}_{1}\right)}{2}\right\}=\frac{\delta\left(\dot{g}_{1}\right)}{2}$. In this case, the assertion (1) of the previous proposition can be restated as follows. $\widetilde{\mathcal{R}}_{\mu, k}^{\ell}$ is $L^{p}$ outside the diagonal of $\Gamma \backslash G / K$ for all integers $k>\frac{1}{4} \operatorname{dim}_{\mathbf{R}}(G / K)$ and $p \geq 1$, and real numbers $\mu<-\alpha_{\ell}$. The assertion (2) may be restated accordingly. 
THEOREM 5.9. Let $G$ be a non compact connected semisimple real Lie group with finite center, $K$ a maximal compact subgroup of $G$ and $\Gamma$ a torsion free discrete subgroup of $G$ with finite covolume. Fix an element $g$ in $G$. Then for all complex number $\mu$ with positive imaginary part, there exists a positive number $\epsilon$ such that $\widetilde{\mathcal{R}}_{\mu, k}^{\ell}(\dot{g}, \cdot) \in L^{2+\epsilon}\left(\Gamma \backslash G / K, \mathcal{E} n d\left(\Lambda^{\ell} \mathfrak{s}\right)\right)$, for all integer $k>\frac{1}{4} \operatorname{dim}_{\mathbf{R}}(G / K)$.

Proof. Throughout the proof $C_{j}$ will denote a positive real number.

Step 1: Let us decompose the Hilbert space $L^{2}\left(\Gamma \backslash G / K, \Lambda^{\ell} \mathfrak{s}\right)$ as follows

$$
L^{2}\left(\Gamma \backslash G / K, \Lambda^{\ell} \mathfrak{s}\right)=\operatorname{Ker}\left(\widetilde{\Delta}_{\ell}\right) \oplus \operatorname{Ker}\left(\widetilde{\Delta}_{\ell}\right)^{\perp}
$$

where $\operatorname{Ker}\left(\widetilde{\Delta}_{\ell}\right)$ denotes the space of square integrable harmonic $\ell$-forms and $\operatorname{Ker}\left(\widetilde{\Delta}_{\ell}\right)^{\perp}$ its orthogonal complement. By a result of A. Borel and H. Garland [9], $\operatorname{Ker}\left(\widetilde{\Delta}_{\ell}\right)$ is finite dimensional for all $\ell$ when $\Gamma$ is of finite covolume. So we may write the orthogonal projection $T_{\ell}: L^{2}\left(\Gamma \backslash G / K, \Lambda^{\ell} \mathfrak{s}\right) \rightarrow \operatorname{Ker}\left(\widetilde{\Delta}_{\ell}\right)$ on $\operatorname{Ker}\left(\widetilde{\Delta}_{\ell}\right)$ as

$$
T_{\ell}=\sum_{j}\left\langle\varphi_{j}, \cdot\right\rangle \varphi_{j}
$$

where $\left\{\varphi_{j}\right\}$ is some orthonormal basis of $\operatorname{Ker}\left(\widetilde{\Delta}_{\ell}\right)$.

Step 2: By Lemma 2 of [23], there exists a positive number $\epsilon_{0}$ such that

$$
\varphi_{j} \in L^{2+\epsilon}\left(\Gamma \backslash G / K, \Lambda^{\ell} \mathfrak{s}\right) \quad \forall \epsilon \in\left[0, \epsilon_{0}[.\right.
$$

It turns out that $T_{\ell}$ is a bounded operator on $L^{2+\epsilon}\left(\Gamma \backslash G / K, \Lambda^{\ell} \mathfrak{s}\right)$ for all $\epsilon \in\left[0, \epsilon_{0}[\right.$. Indeed let $f \in L^{2+\epsilon}\left(\Gamma \backslash G / K, \Lambda^{\ell} \mathfrak{s}\right)$ and write $\epsilon^{\prime}$ for the conjugate $\frac{2+\epsilon}{1+\epsilon}$ of $2+\epsilon$. Since $\Gamma$ has finite covolume in $G$, the Hölder inequality implies that

$$
\varphi_{j} \in L^{q}\left(\Gamma \backslash G / K, \Lambda^{\ell} \mathfrak{s}\right) \forall q \in[1,2] .
$$

In particular, $\varphi_{j} \in L^{\epsilon^{\prime}}\left(\Gamma \backslash G / K, \Lambda^{\ell} \mathfrak{s}\right)$ and $\left\langle\varphi_{j}, f\right\rangle \in L^{1}(\Gamma \backslash G / K)$. Observe that $T_{\ell}$ is also a bounded operator on $L^{\epsilon^{\prime}}\left(\Gamma \backslash G / K, \Lambda^{\ell} \mathfrak{s}\right)$ by selfadjointness.

Step 3: Let $w$ be a complex number with positive imaginary part. Fix a real number $c \in] 0,1$ [ and let $B_{c}$ be the ball of radius $c$ centered at the origin. We have

$$
\begin{aligned}
& \int_{\left\|g_{0}\right\|>B_{c}}\left\|\mathcal{R}_{w}^{\ell}\left(g, g_{0}\right)\right\|_{\operatorname{End}\left(\Lambda^{\ell} \mathfrak{s}\right)} d g_{0} \\
\leq & b_{c} \int_{\left\|a^{+}\left(g_{0}\right)\right\|>B_{c}} \Phi_{0}\left(a^{+}\left(g_{0}\right)\right) e^{-(1-c) \tau_{w, \ell}(G / K)\left\|a^{+}\left(g_{0}\right)\right\|} d g_{0} \text { by }(4.2) \\
\leq & C_{1} \int_{\left\|a^{+}\left(g_{0}\right)\right\|>B_{c}}\left(\Pi_{\alpha \in \Sigma^{++}}\left(1+\alpha\left(a^{+}\left(g_{0}\right)\right)\right)\right) e^{-\rho_{\mathfrak{a}_{0}}\left(a^{+}\left(g_{0}\right)\right)} e^{-(1-c) \tau_{w, \ell}(G / K)\left\|a^{+}\left(g_{0}\right)\right\|} d g_{0}
\end{aligned}
$$

by $(2.19)$

$\leq C_{2} \int_{\mathfrak{a}_{0}^{+}}\left(\Pi_{\alpha \in \Sigma^{++}}(1+\alpha(X))\right) e^{\rho_{a_{0}}(X)-(1-c) \tau_{w, \ell}(G / K)\|X\|} d X$

by integration formula (Prop. 5.28 of [20]).

The latter integral is finite if

$$
\begin{aligned}
& \rho_{\mathfrak{a}_{0}}(X)<(1-c) \tau_{w, \ell}(G / K)\|X\|, \quad \forall X \in \mathfrak{a}_{0}^{+} \\
\Leftrightarrow & \tau_{w, \ell}(G / K)>\frac{1}{1-c} \rho_{\mathfrak{a}_{0}}(Y), \quad \forall Y \in \mathfrak{a}_{0}^{+},\|Y\|=1 \\
\Leftrightarrow & \lambda_{\ell}(G / K)-\operatorname{Re}(w)+\sqrt{\left(\lambda_{\ell}(G / K)-\operatorname{Re}(w)\right)^{2}+\operatorname{Im}(w)^{2}}>\frac{2}{(1-c)^{2}} \rho_{\text {max }}^{2}
\end{aligned}
$$


where $\rho_{\max }$ is the positive real number defined by

$$
\rho_{\max }=\sup \left\{\rho_{\mathfrak{a}_{0}}(Y) \mid Y \in \mathfrak{a}_{0}^{+},\|Y\|=1\right\} .
$$

Moreover, Proposition 5.2 (2)(ii) shows that the integral

$$
\int_{\left\|g_{0}\right\| \leq B_{c}}\left\|\mathcal{R}_{w}^{\ell}\left(g, g_{0}\right)\right\|_{\operatorname{End}\left(\Lambda^{\ell} \mathfrak{s}\right)} d g_{0}
$$

is finite for all complex number $w$ with positive imaginary part. On the other hand, observe that for all $\phi \in L^{2}\left(\Gamma \backslash G / K, \Lambda^{\ell} \mathfrak{s}\right)$, one has

$$
\begin{aligned}
\left\|\left(\widetilde{R}_{w}^{\ell} \phi\right)(\dot{g})\right\|_{\Lambda^{\ell_{\mathfrak{S}}}} & =\left\|\int_{\Gamma \backslash G} \widetilde{\mathcal{R}}_{w}^{\ell}\left(\dot{g}, \dot{g}_{0}\right) \phi\left(\dot{g}_{0}\right) d \dot{g}_{0}\right\|_{\Lambda^{\ell} \mathfrak{s}} \\
& =\left\|\int_{\Gamma \backslash G} \sum_{\gamma \in \Gamma} \mathcal{R}_{w}^{\ell}\left(g^{-1} \gamma g_{0}\right) \phi\left(\dot{g}_{0}\right) d \dot{g}_{0}\right\|_{\Lambda^{\ell_{\mathfrak{S}}}} \\
& \leq\|\phi\|_{L^{1}\left(\Gamma \backslash G / K, \Lambda^{\left.\mathfrak{s}_{\mathfrak{s}}\right)}\right.} \int_{\Gamma \backslash G} \sum_{\gamma \in \Gamma}\left\|\mathcal{R}_{w}^{\ell}\left(g^{-1} \gamma g_{0}\right)\right\|_{\operatorname{End}\left(\Lambda^{\ell} \mathfrak{s}\right)} d \dot{g}_{0} \\
& =\|\phi\|_{L^{1}\left(\Gamma \backslash G / K, \Lambda^{\left.\mathfrak{e}_{\mathfrak{s}}\right)}\right.} \int_{G}\left\|\mathcal{R}_{w}^{\ell}\left(g, g_{0}\right)\right\|_{\operatorname{End}\left(\Lambda^{\ell_{\mathfrak{s}}}\right)} d g_{0} .
\end{aligned}
$$

Thus $\widetilde{R}_{w}^{\ell}$ is a bounded operator on $L^{1}\left(\Gamma \backslash G / K, \Lambda^{\ell} \mathfrak{s}\right)$ if

$$
\operatorname{Im}(w)^{2}>\left(\frac{2}{(1-c)^{2}} \rho_{\text {max }}^{2}-\left(\lambda_{\ell}(G / K)-\operatorname{Re}(w)\right)\right)^{2}-\left(\lambda_{\ell}(G / K)-\operatorname{Re}(w)\right)^{2} .
$$

Recall that, by definition, $\widetilde{R}_{w}^{\ell}$ is a bounded operator on $L^{2}\left(\Gamma \backslash G / K, \Lambda^{\ell} \mathfrak{s}\right)$ if $\operatorname{Im}(w)>0$.

Step 4: Let $e_{0}$ be the smallest non zero eigenvalue of $\widetilde{\Delta}_{\ell}$ and $z$ a complex number. Then $\widetilde{R}_{z+e_{0}}^{\ell} \circ\left(11-T_{\ell}\right)$ is a bounded operator $L^{2}\left(\Gamma \backslash G / K, \Lambda^{\ell} \mathfrak{s}\right)$ if $\operatorname{Im}(z)>0$, and, from the previous step, $\widetilde{R}_{z+e_{0}}^{\ell}$ is bounded on $L^{1}\left(\Gamma \backslash G / K, \Lambda^{\ell} \mathfrak{s}\right)$ if

$$
\operatorname{Im}(z)^{2}>\left(\frac{2}{(1-c)^{2}} \rho_{\text {max }}^{2}-\left(\lambda_{\ell}(G / K)-e_{0}-\operatorname{Re}(z)\right)\right)^{2}-\left(\lambda_{\ell}(G / K)-e_{0}-\operatorname{Re}(z)\right)^{2} .
$$

Therefore, for all $f \in L^{\epsilon^{\prime}}\left(\Gamma \backslash G / K, \Lambda^{\ell} \mathfrak{s}\right)$, one has

$$
\begin{aligned}
\left\|\widetilde{R}_{z+e_{0}}^{\ell} \circ\left(11-T_{\ell}\right) f\right\|_{L^{\epsilon^{\prime}}\left(\Gamma \backslash G / K, \Lambda^{\ell} \mathfrak{s}\right)} & \leq C_{3}\left\|\left(\mathbb{1}-T_{\ell}\right) f\right\|_{L^{\epsilon^{\prime}}\left(\Gamma \backslash G / K, \Lambda^{\ell} \mathfrak{s}\right)} \\
& \leq\|f\|_{L^{\epsilon^{\prime}}\left(\Gamma \backslash G / K, \Lambda^{\ell} \mathfrak{s}\right)}
\end{aligned}
$$

i.e $\widetilde{R}_{z+e_{0}}^{\ell} \circ\left(11-T_{\ell}\right)$ is a bounded operator $L^{\epsilon^{\prime}}\left(\Gamma \backslash G / K, \Lambda^{\ell} \mathfrak{s}\right)$. Now, using the Stein interpolation theorem (Theorem V.4.1 in [35]), we deduce that $\widetilde{R}_{z+e_{0}}^{\ell} \circ\left(11-T_{\ell}\right)$ is a bounded operator on $L^{p}\left(\Gamma \backslash G / K, \Lambda^{\ell} \mathfrak{s}\right)$ for $\epsilon^{\prime} \leq p \leq 2$ and $\operatorname{Im}(z)$ satisfying

$$
\operatorname{Im}(z)>\theta \sqrt{\left(\frac{2}{(1-c)^{2}} \rho_{\text {max }}^{2}-\left(\lambda_{\ell}(G / K)-e_{0}-\operatorname{Re}(z)\right)\right)^{2}-\left(\lambda_{\ell}(G / K)-e_{0}-\operatorname{Re}(z)\right)^{2}}
$$

where $\frac{1}{p}=\frac{1-\theta}{\epsilon^{\prime}}+\frac{\theta}{2}$ with $\theta \in[0,1]$ and $\left.c \in\right] 0,1[$. Finally, observing that

$$
\left(\left(\widetilde{\Delta}_{\ell}-e_{0}-z\right)^{-1} \circ T_{\ell}\right)(f)=-\frac{1}{z+e_{0}} T_{\ell}(f) \quad \forall f \in L^{p}\left(\Gamma \backslash G / K, \Lambda^{\ell} \mathfrak{s}\right)
$$


and writing

$$
\left(\widetilde{\Delta}_{\ell}-e_{0}-z\right)^{-1}=\left(\widetilde{\Delta}_{\ell}-e_{0}-z\right)^{-1} \circ T_{\ell}+\left(\widetilde{\Delta}_{\ell}-e_{0}-z\right)^{-1} \circ\left(\mathbb{1}-T_{\ell}\right),
$$

we see that $\widetilde{R}_{z+e_{0}}^{\ell}$ is a bounded operator on $L^{p}\left(\Gamma \backslash G / K, \Lambda^{\ell} \mathfrak{s}\right)$. In particular, $\widetilde{R}_{z+e_{0}}^{\ell}$ is bounded on $L^{p}\left(\Gamma \backslash G / K, \Lambda^{\ell} \mathfrak{s}\right)$.

Step 5: Let $\mu_{0}$ be a real number satisfying $\mu_{0}+\alpha_{\ell}<0$ and $\mu$ a complex number with $\operatorname{Im}(\mu)>0$. Recall that, from formulas (5.4) and (5.7), one has

$$
\widetilde{\mathcal{R}}_{\mu, k}^{\ell}=\sum_{j=0}^{k} C_{k}^{j}\left(\mu-\mu_{0}\right)^{N j} \widetilde{\mathcal{R}}_{\mu_{0}, N j}^{\ell} \circ \widetilde{\mathcal{R}}_{\mu, j}^{\ell} \circ\left[\sum_{r=1}^{N}\left(\mu-\mu_{0}\right)^{r} \widetilde{\mathcal{R}}_{\mu_{0}, r}^{\ell}\right]^{k-j}
$$

where the generic term is of the form $\widetilde{\mathcal{R}}_{\mu_{0}, N j}^{\ell} \circ \widetilde{\mathcal{R}}_{\mu, j}^{\ell} \circ \widetilde{\mathcal{R}}_{\mu_{0}, r(k-j)}^{\ell}$. Then it is enough to consider the case where $j=r=1$. By the semigroup property, we have

$$
\widetilde{\mathcal{R}}_{\mu}^{\ell}\left(\dot{g}_{1}, \cdot\right) \widetilde{\mathcal{R}}_{\mu_{0}, k+N-1}^{\ell}\left(\dot{g}_{2}, \cdot\right)=\int_{\Gamma \backslash G} \widetilde{\mathcal{R}}_{\mu}^{\ell}\left(\dot{g}_{1}, \dot{g}_{3}\right) \circ \widetilde{\mathcal{R}}_{\mu_{0}, k+N-1}^{\ell}\left(\dot{g}_{3}, \dot{g}_{2}\right) d \dot{g}_{3} .
$$

But, by proposition 5.5(1), we know that $\widetilde{\mathcal{R}}_{\mu_{0}, k}^{\ell}\left(\dot{g}_{1}, \cdot\right)$ is $L^{q}$ outside any ball centered at $\dot{g}_{1}$ with finite radius, for all $q \geq 2$. Therefore $\widetilde{\mathcal{R}}_{\mu, k}^{\ell}\left(\dot{g}_{1}, \cdot\right)$ is $L^{p}$ on such neighborhoods if $\operatorname{Im}(\mu)$ satisfies the condition

$$
\operatorname{Im}(\mu)>\theta \sqrt{\left(\frac{2}{(1-c)^{2}} \rho_{\text {max }}^{2}-\left(\lambda_{\ell}(G / K)-\operatorname{Re}(\mu)\right)\right)^{2}-\left(\lambda_{\ell}(G / K)-\operatorname{Re}(\mu)\right)^{2}} .
$$

Finally, in the case where

$$
0<\operatorname{Im}(\mu) \leq \theta \sqrt{\left(\frac{2}{(1-c)^{2}} \rho_{\text {max }}^{2}-\left(\lambda_{\ell}(G / K)-\operatorname{Re}(\mu)\right)\right)^{2}-\left(\lambda_{\ell}(G / K)-\operatorname{Re}(\mu)\right)^{2}}
$$

we apply the previous result, replacing $\theta$ by $\theta^{\prime}$ with

$$
0<\theta^{\prime}<\operatorname{Im}(\mu)\left[\left(\frac{2}{(1-c)^{2}} \rho_{\max }^{2}-\left(\lambda_{\ell}(G / K)-\operatorname{Re}(\mu)\right)\right)^{2}-\left(\lambda_{\ell}(G / K)-\operatorname{Re}(\mu)\right)^{2}\right]^{-\frac{1}{2}}
$$

and $\frac{1}{p}=\frac{1-\theta^{\prime}}{\epsilon^{\prime}}+\frac{\theta^{\prime}}{2}$.

REMARK 5.10. Using the same argument as above, one can show that the resolvent $R_{\mu}^{\ell}$ of $\Delta_{\ell}$ is a bounded operator on $L^{q}\left(G / K, \Lambda^{\ell} \mathfrak{s}\right)$ for $q \geq 2$ and

$$
\operatorname{Im}(\mu)>\theta \sqrt{\left(\frac{2}{(1-c)^{2}} \rho_{\text {max }}^{2}-\left(\lambda_{\ell}(G / K)-\operatorname{Re}(\mu)\right)\right)^{2}-\left(\lambda_{\ell}(G / K)-\operatorname{Re}(\mu)\right)^{2}} .
$$

where $q=\frac{2}{1-\theta}$ with $\theta \in[0,1[$ and $c \in] 0,1[$. 


\section{Lower bounds for the bottom of the spectrum of $\widetilde{\Delta}_{\ell}$ and $L^{2}$ - cohomology of $\Gamma \backslash G / K$.}

THEOREM 6.1. Let $G$ be a non compact connected semisimple real Lie group with finite center, $K$ a maximal compact subgroup of $G$ and $\Gamma$ a torsion free discrete subgroup of $G$. We assume that $\Gamma$ is of infinite covolume in $G$ and that the bottom of the spectrum of $\Delta_{\ell}$ does not vanish. Then we have

(i) if $\delta(\Gamma) \leq \rho_{\text {min }}$ then $\beta_{\ell}(\Gamma \backslash G / K) \geq \lambda_{\ell}(G / K)$,

(ii) if $\rho_{\min } \leq \delta(\Gamma) \leq\left\|\rho_{\mathfrak{a}_{0}}\right\|+\sqrt{\lambda_{\ell}(G / K)}$ then $\beta_{\ell}(\Gamma \backslash G / K) \geq \lambda_{\ell}(G / K)-(\delta(\Gamma)-$ $\left.\rho_{\min }\right)^{2}$, and

(iii) if $\left|\delta(\Gamma)-\rho_{\text {min }}\right| \leq\left\|\rho_{\mathfrak{a}_{0}}\right\|<\delta(\Gamma)$ and $\lambda_{\ell}(G / K) \geq\left(\delta(\Gamma)-\rho_{\text {min }}\right)^{2}$ then

$$
\beta_{\ell}(\Gamma \backslash G / K) \geq \lambda_{\ell}(G / K)-\left(\delta(\Gamma)-\rho_{\min }\right)^{2} .
$$

Proof. Throughout the proof the symbols $B_{j}$ will denote positive real numbers. Let $\theta$ be a smooth function on $G$ defined by $\theta(g)=1$ for $\|g\| \leq 1$ and $\theta(g)=0$ for \| $g \|$ sufficiently large. For a real number $\mu<\lambda_{\ell}(G / K)$, decompose the kernel $\mathcal{R}_{\mu}^{\ell}$ of the resolvent $\left(\Delta_{\ell}-\mu\right)^{-1}$ as follows

$$
\mathcal{R}_{\mu}^{\ell}=\mathcal{R}_{\mu}^{\ell, 1}+\mathcal{R}_{\mu}^{\ell, 2}
$$

where $\mathcal{R}_{\mu}^{\ell, 1}=\theta \mathcal{R}_{\mu}^{\ell}$ and $\mathcal{R}_{\mu}^{\ell, 2}=(1-\theta) \mathcal{R}_{\mu}^{\ell}$. Accordingly the kernel $\widetilde{\mathcal{R}}_{\mu}^{\ell}$ of the resolvent $\left(\widetilde{\Delta}_{\ell}-\mu\right)^{-1}$ decomposes as

$$
\widetilde{\mathcal{R}}_{\mu}^{\ell}=\widetilde{\mathcal{R}}_{\mu}^{\ell, 1}+\widetilde{\mathcal{R}}_{\mu}^{\ell, 2} .
$$

Now we have, using (2.39)

$$
\begin{aligned}
\int_{\Gamma \backslash G}\left\|\widetilde{\mathcal{R}}_{\mu}^{\ell, 1}\left(\dot{g}_{1}, \dot{g}_{2}\right)\right\|_{\operatorname{End}\left(\Lambda^{\ell} \mathfrak{s}\right)} d \dot{g}_{2} & =\int_{\Gamma \backslash G}\left\|\sum_{\gamma \in \Gamma} \mathcal{R}_{\mu}^{\ell, 1}\left(g_{1}^{-1} \gamma g_{2}\right)\right\|_{\operatorname{End}\left(\Lambda^{\ell} \mathfrak{s}\right)} d \dot{g}_{2} \\
& =\int_{\Gamma \backslash G} \sum_{\gamma \in \Gamma}\left\|\mathcal{R}_{\mu}^{\ell, 1}\left(g_{1}^{-1} \gamma g_{2}\right)\right\|_{\operatorname{End}\left(\Lambda^{\ell} \mathfrak{s}\right)} d \dot{g}_{2} \\
& =\int_{G}\left\|\mathcal{R}_{\mu}^{\ell, 1}\left(g_{1}, g_{2}\right)\right\|_{\operatorname{End}\left(\Lambda^{\ell} \mathfrak{s}\right)} d g_{2} .
\end{aligned}
$$

Since $\mathcal{R}_{\mu}^{\ell, 1}$ is integrable on the unit ball of $G$, by Proposition 5.2 (2)(ii), we deduce that, for all $\phi$ in $L^{2}\left(\Gamma \backslash G / K, \Lambda^{\ell} \mathfrak{s}\right)$

$$
\left\|\widetilde{R}_{\mu}^{\ell, 1} \phi\right\|_{L^{2}\left(\Gamma \backslash G / K, \Lambda^{\ell} \mathfrak{s}\right)} \leq B_{1}\|\phi\|_{L^{2}\left(\Gamma \backslash G / K, \Lambda^{\ell} \mathfrak{s}\right)} .
$$

Next choose a real number $\mu_{\epsilon}$ such that

$$
\left\|\rho_{\mathfrak{a}_{0}}\right\|^{2}-(1-\epsilon)^{2}\left(\lambda_{\ell}(G / K)-\mu\right) \leq \mu_{\epsilon} \leq\left\|\rho_{\mathfrak{a}_{0}}\right\|^{2}
$$

for some $\epsilon \in] 0,1[$. Then, combining Theorem 4.1 and (5.1), there exists a positive constant $C_{\mu_{\epsilon}}$, depending on $\epsilon$, such that

$$
\mathcal{R}_{\mu}^{\ell, 2}(g, \cdot) \leq C_{\mu_{\epsilon}} \mathcal{S}_{\mu_{\epsilon}}(g, \cdot) \text { for }\|g\| \text { sufficiently large. }
$$

In particular we have

$$
\begin{aligned}
\widetilde{\mathcal{R}}_{\mu}^{\ell, 2}\left(\dot{g}_{1}, \dot{g}_{2}\right) & =\sum_{\gamma \in \Gamma} \mathcal{R}_{\mu}^{\ell, 2}\left(g_{1}^{-1} \gamma g_{2}\right) \\
& \leq C_{\mu_{\epsilon}} \sum_{\gamma \in \Gamma} \mathcal{S}_{\mu_{\epsilon}}\left(g_{1}^{-1} \gamma g_{2}\right) \\
& =C_{\mu_{\epsilon}} \widetilde{\mathcal{S}}_{\mu_{\epsilon}}\left(\dot{g}_{1}, \dot{g}_{2}\right)
\end{aligned}
$$


so that

$$
\begin{aligned}
\left\|\left(\widetilde{R}_{\mu}^{\ell, 2} \phi\right)\left(\dot{g}_{1}\right)\right\|_{\Lambda^{\ell_{\mathfrak{s}}}} & =\left\|\int_{\Gamma \backslash G} \widetilde{\mathcal{R}}_{\mu}^{\ell, 2}\left(\dot{g}_{1}, \dot{g}_{2}\right) \phi\left(\dot{g}_{2}\right) d \nu\left(\dot{g}_{2}\right)\right\|_{\Lambda^{\ell_{\mathfrak{s}}}} \\
& \leq C_{\mu_{\epsilon}} \int_{\Gamma \backslash G} \widetilde{\mathcal{S}}_{\mu_{\epsilon}}\left(\dot{g}_{1}, \dot{g}_{2}\right)\left\|\phi\left(\dot{g}_{2}\right)\right\|_{\Lambda^{\ell_{\mathfrak{s}}}} d \nu\left(\dot{g}_{2}\right) .
\end{aligned}
$$

Now, by the assertion (i) of Leuzinger's theorem (see Section 2.11), we know that $\widetilde{S}_{\mu_{\epsilon}}$ is bounded on $L^{2}(\Gamma \backslash G / K)$, so that

$$
\left\|\widetilde{R}_{\mu}^{\ell, 2} \phi\right\|_{L^{2}\left(\Gamma \backslash G / K, \Lambda^{\ell} \mathfrak{s}\right)} \leq B_{2}\|\phi\|_{L^{2}\left(\Gamma \backslash G / K, \Lambda^{\ell} \mathfrak{s}\right)} .
$$

Finally, we deduce, from (6.2), (6.3) and (6.4), that $R_{\mu}^{\ell}$ is bounded on $L^{2}\left(\Gamma \backslash G / K, \Lambda^{\ell} \mathfrak{s}\right)$

$$
\left\|R_{\mu}^{\ell} \phi\right\|_{L^{2}\left(\Gamma \backslash G / K, \Lambda^{\ell} \mathfrak{s}\right)} \leq B_{3}\|\phi\|_{L^{2}\left(\Gamma \backslash G / K, \Lambda^{\ell} \mathfrak{s}\right)} .
$$

Therefore $\beta_{\ell}(\Gamma \backslash G / K) \geq \lambda_{\ell}(G / K)$, which proves (i). The proof of (ii) and (iii) is very similar.

For (ii) we choose $\mu<\lambda_{\ell}(G / K)-\left(\delta(\Gamma)-\rho_{\min }\right)^{2}$ and

$$
\left\|\rho_{\mathfrak{a}_{0}}\right\|^{2}-(1-\epsilon)^{2}\left(\lambda_{\ell}(G / K)-\mu\right) \leq \mu_{\epsilon} \leq\left\|\rho_{\mathfrak{a}_{0}}\right\|^{2}-\left(\delta(\Gamma)-\rho_{\text {min }}\right)^{2} .
$$

For (iii) we choose $\mu<\lambda_{\ell}(G / K)-\left(\delta(\Gamma)-\rho_{\text {min }}\right)^{2}$ and

$$
\left\|\rho_{\mathfrak{a}_{0}}\right\|^{2}-(1-\epsilon)^{2}\left(\lambda_{\ell}(G / K)-\mu\right) \leq \mu_{\epsilon} \leq \sup \left\{0 ;\left\|\rho_{\mathfrak{a}_{0}}\right\|^{2}-\left(\delta(\Gamma)-\rho_{\min }\right)^{2}\right\} .
$$

CoROLlary 6.5. Under the assumptions of the previous theorem, the (reduced or unreduced) $L^{2}$-cohomology group of degree $\ell$ of $\Gamma \backslash G / K$ vanishes in the following cases:

(i) $\delta(\Gamma) \leq \rho_{\min }$,

(ii) $\rho_{\text {min }} \leq \delta(\Gamma) \leq\left\|\rho_{\mathfrak{a}_{0}}\right\|+\sqrt{\lambda_{\ell}(G / K)}$ and $\sqrt{\lambda_{\ell}(G / K)}>\delta(\Gamma)-\rho_{\text {min }}$, and

(iii) $\left|\delta(\Gamma)-\rho_{\text {min }}\right| \leq\left\|\rho_{\mathfrak{a}_{0}}\right\|<\delta(\Gamma)$ and $\sqrt{\lambda_{\ell}(G / K)}>\left|\delta(\Gamma)-\rho_{\min }\right|$.

In particular, in these cases, the kernel of $\widetilde{\Delta}_{\ell}$ is reduced to $\{0\}$.

Proof. We deduce from the previous theorem that, in each case (i)-(ii)-(iii), any square integrable closed $\ell$-form on $\Gamma \backslash G / K$ is exact. In other words, the unreduced $L^{2}$-cohomology group $H^{(\ell)}(\Gamma \backslash G / K)$ of degree $\ell$ is trivial, and therefore the reduced $L^{2}$-cohomology group $\bar{H}^{(\ell)}(\Gamma \backslash G / K)$ vanishes as well.

Finally, to sum up, we can say that using algebraic and analytic tools from representation theory of semisimple Lie groups, we obtained estimates for large time behavior of the heat kernel for dfferential forms on symmetric spaces of the type $G / K$, where $G$ is a non compact connected semisimple Lie group with finite center and $K$ a maximal compact subgroup of $G$ (Theorem 3.1). Then, combining these estimates with some techniques from the theory of special functions, we deduced estimates for the resolvent of the form Laplacian on $G / K$ (Theorem 4.1). As a byproduct, we obtained $L^{2+\epsilon}$-estimates for the resolvent of the form Laplacian $\widetilde{\Delta}_{\ell}$ on locally symmetric spaces $\Gamma \backslash G / K$ when $\Gamma$ is a torsion free discrete subgroup of $G$ with finite covolume 
(Theorem 5.9). The latter estimates play an important role in the theory of "Eisenstein transforms" and the Langlands' decomposition of $L^{2}(\Gamma \backslash G / K)$ (see Theorems 4.2 and 4.7 in [30]). As an application of these $L^{2+\epsilon}$-estimates, we derived lower bounds for the bottom of the spectrum of $\widetilde{\Delta}_{\ell}$ when $\Gamma$ has infinite covolume (Theorem 6.1 ) and a vanishing criterion for $L^{2}$-cohomology of $\Gamma \backslash G / K$ (Corollary 6.5). We also mention that, after our results were announced in [25], G. Carron posted a preprint on his webpage in which he proves, using techniques rather different from ours, analogous estimates for the Green kernel and the heat kernel for Laplacian-type operators on symmetric spaces [12].

\section{Appendix A. On the computation of $\lambda_{\ell}(G / K)$.}

A.1. The complex case. Let $G$ be a connected complex semisimple Lie group and $K$ a compact real form of $G$. If $\mathfrak{b}_{0}$ is a maximal abelian subspace in the Lie algebra $\mathfrak{k}_{0}$ of $K$ then the complexification of $\mathfrak{b}_{0}+\sqrt{-1} \mathfrak{b}_{0}$ is a Cartan subalgebra of $\mathfrak{g}$. Write $\Delta=\Delta(\mathfrak{g}, \mathfrak{h})$ for the set of $\mathfrak{g}$-roots relative to $\mathfrak{h}$. If we define the following real number

$$
C_{\ell}(G)=\sup \left\{\left\|<Q>_{\mathfrak{b}_{0}}\right\|^{2}\left|Q \subset \Delta, \sup \left\{0 ; \ell-\operatorname{rk}_{\mathbf{C}}(G)\right\} \leq\right| Q \mid \leq \ell\right\}
$$

where $\left\langle Q>_{\mathfrak{b}_{0}}\right.$ denotes the restriction to $\mathfrak{b}_{0}$ of the sum of elements in the subset $Q$ and $|Q|$ is the number of elements in $Q$, then we have

$$
\lambda_{\ell}(G / K)=\frac{1}{12} \operatorname{dim}_{\mathbf{R}}(G / K)-C_{\ell}(G)
$$

A.2. The real case. We consider the Hermitian spaces $G / K=$ $S O_{e}(2, n) / S O(2) \times S O(n)$ with $n \geq 2$. Recall from Section 2, if $\pi_{P_{i}, \delta_{i}, \sqrt{-1} \nu_{i}}$ is the principal series representation associated with a cuspidal parabolic subgroup $P_{i}=M_{i} A_{i} N_{i}$ of $G, \delta_{i}$ a discrete series representation of $M_{i}$ and $\nu_{i}$ a linear form on $\mathfrak{a}_{i, 0}$, then the Casimir operator of $G$ acts on the (smooth vectors of the) $\pi_{P_{i}, \delta_{i}, \sqrt{-1} \nu_{i}}$ as the scalar operator $\omega_{P_{i}, \delta_{i}, \sqrt{-1}} \nu_{i}$ Id where $\omega_{P_{i}, \delta_{i}, \sqrt{-1} \nu_{i}}$ is the real number $\left\|\operatorname{char}\left(\delta_{i}\right)\right\|^{2}-\left\|\nu_{i}\right\|^{2}-\left\|\rho_{i}\right\|^{2}$ defined by (2.4). In particular, to compute the numbers $\lambda_{\ell}(G / K)$, defined by (2.31), we may assume that $\nu_{i}=0$. If $\lambda$ is the minimal $M_{i} \cap K$-type of $\delta_{i}$ then the infinitesimal character $\operatorname{char}\left(\delta_{i}\right)$ of $\delta_{i}$ is given by (see p. 310 of [20]):

$$
\operatorname{char}\left(\delta_{i}\right)=\lambda-\rho\left(\mathfrak{m}_{i}\right)+2 \rho\left(\mathfrak{m}_{i} \cap \mathfrak{k}\right)
$$

where $\rho\left(\mathfrak{m}_{i}\right)$ (resp. $\left.\rho\left(\mathfrak{m}_{i} \cap \mathfrak{k}\right)\right)$ is the half sum of positive roots of $\mathfrak{m}_{i}$ (resp. $\left.\mathfrak{m}_{i} \cap \mathfrak{k}\right)$ relative to $\mathfrak{t}_{i}$. Here $\mathfrak{t}_{i}$ is a Cartan subalgebra of $\mathfrak{m}$ such that $\mathfrak{h}_{i}=\mathfrak{t}_{i} \oplus \mathfrak{a}_{i}$ is a Cartan subalgebra of $\mathfrak{g}$. Under the Cayley transform, $\mathfrak{h}_{i}$ becomes a compact Cartan subalgebra of $\mathfrak{g}$, and the roots in $\Delta_{i}$ transform accordingly (see p. 417 of [20]). Note that when $M_{i}$ is compact then $\delta_{i}$ is just a highest weight representation with highest weight $\lambda$ and infinitesimal character $\lambda+\rho\left(\mathfrak{m}_{i}\right)$.

1) $G / K=S O_{e}(2,2 n) / S O(2) \times S O(2 n), n \geq 1$.

By Hodge isomorphism $\lambda_{\ell}\left(S O_{e}(2,2 n) / S O(2) \times S O(2 n)\right)=$
$\lambda_{4 n-\ell}\left(S O_{e}(2,2 n) / S O(2) \times S O(2 n)\right)$ for $0 \leq \ell \leq 4 n$, so we may restrict our
attention to $0 \leq \ell \leq 2 n$.
Up to a conjugacy, we need only to consider the minimal parabolic with $M_{i}=$
$M=M \cap K \simeq S O(2 n-2)$. Using branching laws for $S O(2 n) \rightarrow S O(2 n-1)$
and $S O(2 n-1) \rightarrow S O(2 n-2)$ (Theorems 8.1 .3 and 8.1 .4 of [17]) along with


the explicit decomposition of the isotropy representation for the Hermitian groups given in [31], one finds that

$$
\begin{aligned}
n=1: & \lambda_{\ell}\left(S O_{e}(2,2 n) / S O(2) \times S O(2 n)\right)=\frac{1}{4} \text { for } \ell=0,1,2 . \\
n=2: \quad \lambda_{0}\left(S O_{e}(2,2 n) / S O(2) \times S O(2 n)\right) & =\frac{5}{8}, \\
\lambda_{1}\left(S O_{e}(2,2 n) / S O(2) \times S O(2 n)\right) & =\frac{1}{2}, \\
\lambda_{\ell}\left(S O_{e}(2,2 n) / S O(2) \times S O(2 n)\right) & =\frac{1}{8} \text { for } \ell=2,3,4 . \\
n \geq 3: \quad \lambda_{0}\left(S O_{e}(2,2 n) / S O(2) \times S O(2 n)\right) & =\frac{2 n^{2}-2 n+1}{4 n}, \\
\lambda_{1}\left(S O_{e}(2,2 n) / S O(2) \times S O(2 n)\right) & =\frac{2 n^{2}-4 n+4}{4 n}, \\
& \lambda_{2}\left(S O_{e}(2,2 n) / S O(2) \times S O(2 n)\right)=\frac{2 n^{2}-6 n+5}{4 n}, \\
& \lambda_{\ell}\left(S O_{e}(2,2 n) / S O(2) \times S O(2 n)\right)=\frac{1}{4 n}\left(2 n^{2}+\frac{1}{2}+\frac{\ell(\ell-4 n)}{2}\right) \text { for } \ell \geq 3 \\
& \text { and } \ell \text { odd },
\end{aligned}
$$

$$
\lambda_{\ell}\left(S O_{e}(2,2 n) / S O(2) \times S O(2 n)\right)=\frac{1}{4 n}\left(2 n^{2}+2 n+1+\frac{\ell(\ell-4 n-2)}{2}\right)
$$
for $\ell \geq 4$ and $\ell$ even.

2) $\underline{G / K=S O_{e}(2,2 n+1) / S O(2) \times S O(2 n+1), n \geq 1 \text {. }}$

Again $\lambda_{\ell}\left(S O_{e}(2,2 n+1) / S O(2) \times S O(2 n+1)\right)=\lambda_{4 n+2-\ell}\left(S O_{e}(2,2 n+\right.$ 1) $/ S O(2) \times S O(2 n+1))$ for $0 \leq \ell \leq 4 n+2$, by Hodge isomorphism, so we may restrict our attention to $0 \leq \ell \leq 2 n+1$.

Now we need to consider two parabolic subgroups $P_{1}$ and $P_{2}$ with $M_{1}=$ $M_{1} \cap K \simeq S O(2 n-1)$ and $M_{2} \simeq S O_{e}(1,2 n), M_{2} \cap K \simeq S O(2 n)$. In particular, using branching laws for $S O(2 n+1) \rightarrow S O(2 n)$ and $S O(2 n) \rightarrow S O(2 n-1)$

(Theorems 8.1.3 and 8.1.4 of [17]), one finds that

$$
\begin{aligned}
n=1: & \lambda_{0}\left(S O_{e}(2,2 n+1) / S O(2) \times S O(2 n+1)\right)=\frac{3}{8}, \\
\lambda_{\ell}\left(S O_{e}(2,2 n+1) / S O(2) \times S O(2 n+1)\right) & =\frac{1}{24} \text { for } \ell=1,2,3 . \\
n \geq 2: & \lambda_{0}\left(S O_{e}(2,2 n+1) / S O(2) \times S O(2 n+1)\right)=\frac{8 n^{2}+1}{16 n+8}, \\
\lambda_{1}\left(S O_{e}(2,2 n+1) / S O(2) \times S O(2 n+1)\right) & =\frac{8 n^{2}-8 n+9}{16 n+8}, \\
& \lambda_{2}\left(S O_{e}(2,2 n+1) / S O(2) \times S O(2 n+1)\right)=\frac{8 n^{2}-16 n+9}{16 n+8}, \\
& \lambda_{\ell}\left(S O_{e}(2,2 n+1) / S O(2) \times S O(2 n+1)\right)=\frac{1}{4 n+2}\left(2 n^{2}+\frac{1}{4}+\frac{\ell(\ell-4 n)}{2}\right) \\
& \text { for } \ell \leq 2 n, \ell \text { even, }
\end{aligned}
$$




$$
\lambda_{\ell}\left(S O_{e}(2,2 n+1) / S O(2) \times S O(2 n+1)\right)=\frac{1}{4 n+2}\left(2 n^{2}+\frac{7}{4}+\frac{\ell(\ell-4 n)}{2}\right)
$$
for $\ell<2 n-1, \ell$ odd,

$$
\lambda_{\ell}\left(S O_{e}(2,2 n+1) / S O(2) \times S O(2 n+1)\right)=\frac{1}{16 n+8} \text { for } \ell=2 n-1,2 n+1 .
$$

\section{REFERENCES}

[1] P. Albuquerque, Patterson-Sullivan theory in higher rank symmetric spaces, Geom. Funct. Anal., 9 (1999), pp. 1-28.

[2] G. Alexopoulos et N. Lohoué, On the large time behavior of heat kernels on Lie groups, Duke Math. J., 120 (2003), pp. 311-351.

[3] J.-P. ANKER AND L. Ji, Heat kernel and Green function estimates on noncompact symmetric spaces, Geom. Funct. Anal., 9 (1999), pp. 1035-1091.

[4] I. G. Avramidi, Heat Kernel and Quantum Gravity, Springer Verlag, 2000.

[5] E. VAn Den BAn And S. SouAifi, A comparison of Paley-Wiener theorems for real reductive groups, preprint.

[6] D. Barbasch and H. Moscovici, $L^{2}$-index and the Selberg trace formula, J. Funct. Analysis, 53 (1983), pp. 151-201.

[7] N. Berline, E. Getzler and M. Vergne, Heat kernels and Dirac operators, Spinger Verlag, 1991.

[8] A. BorEL, The $L^{2}$-cohomology of negatively curved Riemannian symmetric spaces, Ann. Acad. Scient. Fenn. Series A. I. Math., 10 (1985), pp. 95-105.

[9] A. Borel And H. Garland, Laplacian and the discrete spectrum of an arithmetic group, Amer. J. Math., 105 (1983), pp. 309-335.

[10] A. Borel And N. R. WAllach, Continuous cohomology, discrete subgroups, and representations of reductive groups, Annals of mathematics studies, Princeton University Press, 1980.

[11] R. CAMPOREsi, The Helgason-Fourier transform for homogeneous vector bundles over Riemannian symmetric spaces, Pacific J. Math., 179 (1997), pp. 263-300.

[12] G. CARron, Estimée du noyau de Green et de la chaleur sur les espaces symétriques, preprint 2007. Available at http://www.math.sciences.univ-nantes.fr/ carron/publication.html

[13] G. Carron and E. Pedon, On the differential form spectrum of hyperbolic manifods, Ann. Sc. Norm. Super. Pisa Cl. Sci., 3 (2004), pp. 705-747.

[14] T. Coulhon, L. Saloff-Coste and N. T. Varopoulos, Analysis and geometry on groups, Cambridge Tracts in Mathematics, Vol. 100, Cambridge University Press, 1992.

[15] S. Y. Cheng, P. Li, Peter And S. T. YAu, On the upper estimate of the heat kernel of a complete Riemannian manifold, Amer. J. Math., 103 (1981), pp. 1021-1063.

[16] P. Delorme, Sur le théorème de Paley-Wiener d'Arthur, Ann. of Math., 162 (2005), pp. 9871029.

[17] R. Goodman and N. R. Wallach, Representations and invariants of the classical groups, Encyclopedia of Mathematics and its Applications, Vol. 68, Cambridge University Press, 1998.

[18] Harish-Chandra, Harmonic analysis on semisimple Lie groups, Bull. AMS, 76 (1970), pp. 529-551.

[19] S. Helgason, Geometric analysis on symmetric spaces, Mathematical Surveys and Monographs, Vol. 39, AMS, 1994.

[20] A. W. KnApP, Representation theory of semisimple groups: An overview based on examples, Princeton University Press, Vol. 36, 1986.

[21] E. Leuzinger, Critical exponents of discrete groups and $L^{2}$-spectrum, Proc. of the AMS, 132 (2003), pp. 919-927.

[22] N. Lohoué, Estimation des projecteurs de de Rham-Hodge de certaines variétés Riemanniennes non compactes, Math. Nachr., 279 (2006), pp. 272-298.

[23] N. Lohoué, Stabilité de la cohomologie $L^{p}$ au voisinage de 2 pour les espaces localement symétriques, Ann. Global Anal. Geom., 16 (1998), pp. 543-571.

[24] N. Lohoué, Inégalités de Sobolev pour les sous-laplaciens de certains groupes modulaires, Geom. Funct. Anal., 2 (1992), pp. 394-420.

[25] N. Lohoué ET S. MEhdi, Estimées du noyau de la chaleur pour les formes différentielles sur les espaces symétriques et $L^{2}$-cohomologie des espaces localement symétriques, C. R. Math. Acad. Sci. Paris., 345 (2007), pp. 119-122. 
[26] N. Lohoué And S. Mehdi, The Novikov-Shubin invariants of locally symmetric spaces, J. Math. Pures et App., 79 (2000), pp. 111-140.

[27] J. LotT, The zero-in-spectrum question, Ens. Math., 42 (1996), pp. 341-376.

[28] W. LüCK, $L^{2}$-invariants of regular coverings of compact manifolds and $C W$-complexes, Handbook of geometric topology, pp. 735-817, Editors: Davermann, R.J. and Sher, R.B., Elsevier, 2002.

[29] W. Magnus, F. Oberhettinger and R. P. Soni, Formulas and theorems for the special functions of mathematical physics, Die Grundlehren der mathematischen Wissenschaften, Band 52, Springer-Verlag, 1966.

[30] R. Miatello and N. R. WALlaCh, The resolvent of the Laplacian on locally symmetric spaces, J. Differential Geom., 36 (1992), pp. 663-698.

[31] S. Murakami, Cohomology groups of vector-valued forms on symmetric spaces, Lectures, University of Chicago, 1966.

[32] S. Mustapha, Gaussian estimates for heat kernels on Lie groups, Math. Proc. Cambridge Philos. Soc., 128 (2000), pp. 45-64.

[33] M. Olbrich, $L^{2}$-invariants of locally symmetric spaces, Doc. Math., 7 (2002), pp. 219-237.

[34] P. Ostellari, Global behavior of the heat kernel associated with certain sub-Laplacian on semisimple Lie groups, J. Funct. Analysis, 199 (2003), pp. 521-534.

[35] E. M. Stein and G. Weiss, Introduction to Fourier analysis on euclidian spaces, Princeton Math. Series No. 32, Princeton University Press, 1971.

[36] R. Strichartz, Analysis of the Laplacian on complete Riemannian manifolds, J. Funct. Analysis, 52 (1983), pp. 48-79.

[37] D. V. VAssilevich, Heat kernel expansion: user's manual, Phys. Rept., 388 (2003), pp. 279360.

[38] D. A. Vogan and G. J. Zuckerman, Unitary representations with non-zero cohomology, Comp. Math., 53 (1984), pp. 51-90.

[39] N. R. WALlach, Cyclic vectors and irreducibility for principal series representations, Trans. of the AMS, 158 (1971), pp. 389-396.

[40] N. R. Wallach, Harmonic analysis on homogeneous spaces, Pure and Appl. Math. series, Vol 19, Marcel Dekker, 1973.

[41] D. P. Želobenko, Compact Lie groups and their representations, Translations Math. Monographs, Vol. 40, AMS, 1973. 
N. LOHOUÉ AND S. MEHDI 